Acta Cryst. (2004). C60, 000-000

\title{
Crystal and Molecular Structure of Complex 2
}

DAVIT ZARGARIAN ${ }^{a}$ AND YAOFENG $\mathrm{CHEN}^{b}$

${ }^{a}$ Département de Chimie, Université de Montréal, C.P. 6128, Succ. Centre-ville, Montréal, Québec, Canada, H3C 3J\%, and ${ }^{b}$ Département de Chimie, Université de Montréal, C.P. 6128, Succ.Centre-ville, Montréal, Québec, CanadaH3C 3J7.E-mail:davitz@chimie.umontreal.ca

\section{Abstract}

Here should be written a short abstract

\section{Comment}

Here should be written the text of the article

\section{Experimental}

Small details about the preparation of the compound.

Crystal data

$\mathrm{C}_{30} \mathrm{H}_{30} \mathrm{ClNiPSi}$

$M_{r}=543.76$

Triclinic

$P \overline{1}$

$a=9.9064$ (1)

$b=16.0940$ (1) $\AA$

$c=18.9474(1) \AA$

$\alpha=114.60^{\circ}$

$\beta=90.32^{\circ}$

$\gamma=92.57^{\circ}$

$V=2742.99(4) \AA^{3}$

$Z=4$

$D_{x}=1.317 \mathrm{Mg} \mathrm{m}^{-3}$

$D_{m}$ not measured

$\mathrm{Cu} K \alpha$ radiation

$\lambda=1.54178 \AA$
Cell parameters from 24980 reflections

$\theta=2.57-72.93^{\circ}$

$\mu=2.999 \mathrm{~mm}^{-1}$

$T=223(2) \mathrm{K}$

Block

Red

$0.26 \times 0.24 \times 0.10 \mathrm{~mm}$

Crystal source: synthesized by the authors. See text 
Data collection eter

$\omega$ scans

Absorption correction:

multi-scan Sadabs (Sheldrick,1996)

$T_{\min }=0.5550, T_{\max }=0.8050$

33302 measured reflections

10438 independent reflections
Bruker AXS Smart 2K/Platform diffractom-

8823 reflections with

$$
>2 \operatorname{sigma}(I)
$$

$R_{\text {int }}=0.022$

$\theta_{\max }=72.98^{\circ}$

$h=-11 \rightarrow 12$

$k=-19 \rightarrow 19$

$l=-23 \rightarrow 23$

259 standard reflections

\section{every ? reflections}

intensity decay: $-0.09 \%$

Refinement

Refinement on $F^{2}$

$R\left[F^{2}>2 \sigma\left(F^{2}\right)\right]=0.0454$

$w R\left(F^{2}\right)=0.1301$

$S=1.060$

10438 reflections

898 parameters

$\mathrm{H}$-atom parameters constrained $w=1 /\left[\sigma^{2}\left(F_{o}^{2}\right)+(0.0756 P)^{2}+1.0917 P\right]$

where $P=\left(F_{o}^{2}+2 F_{c}^{2}\right) / 3$

$(\Delta / \sigma)_{\max }=0.001$

$\Delta \rho_{\max }=0.918{\mathrm{e} \AA^{-3}}^{-3}$

$\Delta \rho_{\min }=-0.681$ e $\AA^{-3}$

Extinction correction: none

Scattering factors from International Tables for Crystallography (Vol. C)

Table 1. Selected geometric parameters $\left(\AA,^{\circ}\right)$

$\begin{array}{llll}\text { Ni1-C13 } & 2.052(3) & \mathrm{C} 12-\mathrm{C} 13 & 1.402(4) \\ \text { Ni1-C12 } & 2.070(2) & \mathrm{C} 13-\mathrm{C} 13 \mathrm{a} & 1.451(5) \\ \mathrm{N} 1-\mathrm{C} 11 & 2.128(2) & \mathrm{C} 14-\mathrm{C} 15 & 1.367(6) \\ \mathrm{N} 1-\mathrm{C} 11 & 2.1828(7) & \mathrm{C} 14-\mathrm{C} 13 \mathrm{a} & 1.396(5) \\ \mathrm{N} 1-\mathrm{P} 1 & 2.1886(7) & \mathrm{C} 15-\mathrm{C} 16 & 1.393(6) \\ \mathrm{N} 1-\mathrm{C} 13 \mathrm{a} & 2.323(3) & \mathrm{C} 16-\mathrm{C} 17 & 1.383(4) \\ \mathrm{N} 1-\mathrm{C} 17 \mathrm{a} & 2.335(2) & \mathrm{C} 17-\mathrm{C} 17 \mathrm{a} & 1.392(4) \\ \mathrm{P} 1-\mathrm{C} 151 & 1.796(8) & \mathrm{C} 121-\mathrm{C} 122 & 1.378(3) \\ \mathrm{P} 1-\mathrm{C} 131 & 1.819(4) & \mathrm{C} 121-\mathrm{C} 126 & 1.390(4) \\ \mathrm{P} 1-\mathrm{C} 121 & 1.822(2) & \mathrm{C} 122-\mathrm{C} 123 & 1.386(4) \\ \mathrm{P} 1-\mathrm{C} 141 & 1.851(4) & \mathrm{C} 123-\mathrm{C} 124 & 1.360(5) \\ \mathrm{P} 1-\mathrm{C} 161 & 1.854(8) & \mathrm{C} 124-\mathrm{C} 125 & 1.367(5) \\ \mathrm{S} 11-\mathrm{C} 112 & 1.854(3) & \mathrm{C} 125-\mathrm{C} 126 & 1.382(4) \\ \mathrm{Si1}-\mathrm{C} 113 & 1.860(3) & \mathrm{C} 131-\mathrm{C} 132 & 1.371(5) \\ \mathrm{Si1}-\mathrm{C} 111 & 1.873(3) & \mathrm{C} 131-\mathrm{C} 136 & 1.371(5) \\ \mathrm{Si1}-\mathrm{C} 11 & 1.877(2) & \mathrm{C} 132-\mathrm{C} 133 & 1.379(6) \\ \mathrm{C} 11-\mathrm{C} 12 & 1.423(3) & \mathrm{C} 133-\mathrm{C} 134 & 1.369(7) \\ \mathrm{C} 11-\mathrm{C} 17 \mathrm{a} & 1.471(3) & \mathrm{C} 134-\mathrm{C} 135 & 1.353(9)\end{array}$




\begin{tabular}{|c|c|c|c|}
\hline $\mathrm{C} 135-\mathrm{C} 136$ & $1.387(6)$ & $\mathrm{Si} 2-\mathrm{C} 21$ & $1.871(3)$ \\
\hline $\mathrm{C} 161-\mathrm{C} 162$ & $1.381(7)$ & $\mathrm{C} 21-\mathrm{C} 22$ & $1.415(4)$ \\
\hline $\mathrm{C} 161-\mathrm{C} 166$ & $1.383(7)$ & $\mathrm{C} 21-\mathrm{C} 27 \mathrm{a}$ & $1.466(5)$ \\
\hline $\mathrm{C} 162-\mathrm{C} 163$ & $1.380(8)$ & $\mathrm{C} 22-\mathrm{C} 23$ & $1.410(5)$ \\
\hline $\mathrm{C} 163-\mathrm{C} 164$ & $1.360(18)$ & $\mathrm{C} 23-\mathrm{C} 23 \mathrm{a}$ & $1.449(5)$ \\
\hline C164-C165 & $1.362(17)$ & $\mathrm{C} 24-\mathrm{C} 25$ & $1.361(8)$ \\
\hline $\mathrm{C} 165-\mathrm{C} 166$ & 1.388 & $\mathrm{C} 24-\mathrm{C} 23 \mathrm{a}$ & $1.415(6)$ \\
\hline $\mathrm{C} 141-\mathrm{C} 142$ & $1.382(5)$ & $\mathrm{C} 25-\mathrm{C} 26$ & $1.353(8)$ \\
\hline $\mathrm{C} 141-\mathrm{C} 146$ & $1.392(5)$ & $\mathrm{C} 26-\mathrm{C} 27$ & $1.353(7)$ \\
\hline $\mathrm{C} 142-\mathrm{C} 143$ & $1.384(4)$ & $\mathrm{C} 27-\mathrm{C} 27 \mathrm{a}$ & $1.408(5)$ \\
\hline $\mathrm{C} 143-\mathrm{C} 144$ & $1.365(7)$ & $\mathrm{C} 231-\mathrm{C} 236$ & $1.383(8)$ \\
\hline $\mathrm{C} 144-\mathrm{C} 145$ & $1.373(9)$ & $\mathrm{C} 231-\mathrm{C} 232$ & $1.386(8)$ \\
\hline $\mathrm{C} 145-\mathrm{C} 146$ & $1.394(6)$ & $\mathrm{C} 232-\mathrm{C} 233$ & $1.381(8)$ \\
\hline $\mathrm{C} 151-\mathrm{C} 152$ & $1.355(7)$ & $\mathrm{C} 233-\mathrm{C} 234$ & $1.385(9)$ \\
\hline $\mathrm{C} 151-\mathrm{C} 156$ & $1.357(7)$ & $\mathrm{C} 234-\mathrm{C} 235$ & $1.343(18)$ \\
\hline $\mathrm{C} 152-\mathrm{C} 153$ & $1.376(7)$ & $\mathrm{C} 235-\mathrm{C} 236$ & $1.417(12)$ \\
\hline $\mathrm{C} 153-\mathrm{C} 154$ & $1.354(18)$ & $\mathrm{C} 261-\mathrm{C} 262$ & $1.394(8)$ \\
\hline $\mathrm{C} 154-\mathrm{C} 155$ & $1.360(18)$ & $\mathrm{C} 261-\mathrm{C} 266$ & $1.396(8)$ \\
\hline $\mathrm{C} 155-\mathrm{C} 156$ & $1.386(12)$ & $\mathrm{C} 262-\mathrm{C} 263$ & $1.387(8)$ \\
\hline $\mathrm{Ni} 2-\mathrm{C} 23$ & $2.038(3)$ & $\mathrm{C} 263-\mathrm{C} 264$ & $1.360(17)$ \\
\hline $\mathrm{Ni} 2-\mathrm{C} 22$ & 2.060 & $\mathrm{C} 264-\mathrm{C} 265$ & $1.36(2)$ \\
\hline $\mathrm{Ni} 2-\mathrm{C} 21$ & $2.135(2)$ & $\mathrm{C} 265-\mathrm{C} 266$ & $1.444(16)$ \\
\hline $\mathrm{Ni} 2-\mathrm{Cl} 2 \mathrm{a}$ & $2.1770(11)$ & $\mathrm{C} 271-\mathrm{C} 272$ & 1.338 \\
\hline $\mathrm{Ni} 2-\mathrm{P} 2$ & $2.1819(8)$ & $\mathrm{C} 271-\mathrm{C} 276$ & $1.397(14)$ \\
\hline $\mathrm{Ni} 2-\mathrm{Cl} 2 b$ & $2.195(2)$ & $\mathrm{C} 272-\mathrm{C} 273$ & $1.361(15)$ \\
\hline $\mathrm{Ni} 2-\mathrm{C} 23 \mathrm{a}$ & $2.339(3)$ & $\mathrm{C} 273-\mathrm{C} 274$ & $1.342(18)$ \\
\hline $\mathrm{Ni} 2-\mathrm{C} 27 \mathrm{a}$ & $2.374(3)$ & $\mathrm{C} 274-\mathrm{C} 275$ & $1.349(16)$ \\
\hline $\mathrm{Cl} 2 \mathrm{a}-\mathrm{Cl} 2 b$ & $0.608(4)$ & $\mathrm{C} 275-\mathrm{C} 276$ & $1.411(12)$ \\
\hline $\mathrm{P} 2-\mathrm{C} 291$ & $1.800(8)$ & $\mathrm{C} 281-\mathrm{C} 282$ & $1.380(15)$ \\
\hline $\mathrm{P} 2-\mathrm{C} 271$ & $1.817(6)$ & $\mathrm{C} 281-\mathrm{C} 286$ & $1.405(16)$ \\
\hline $\mathrm{P} 2-\mathrm{C} 231$ & $1.823(7)$ & $\mathrm{C} 282-\mathrm{C} 283$ & $1.384(15)$ \\
\hline $\mathrm{P} 2-\mathrm{C} 241$ & $1.837(5)$ & $\mathrm{C} 283-\mathrm{C} 284$ & $1.362(17)$ \\
\hline $\mathrm{P} 2-\mathrm{C} 281$ & $1.840(8)$ & $\mathrm{C} 284-\mathrm{C} 285$ & $1.35(2)$ \\
\hline $\mathrm{P} 2-\mathrm{C} 251$ & $1.845(7)$ & $\mathrm{C} 285-\mathrm{C} 286$ & $1.414(16)$ \\
\hline $\mathrm{P} 2-\mathrm{C} 261$ & $1.853(8)$ & $\mathrm{C} 241-\mathrm{C} 246$ & $1.376(6)$ \\
\hline $\mathrm{P} 2-\mathrm{C} 221$ & $1.865(6)$ & $\mathrm{C} 241-\mathrm{C} 242$ & $1.378(6)$ \\
\hline $\mathrm{Si} 2-\mathrm{C} 213$ & $1.854(5)$ & $\mathrm{C} 242-\mathrm{C} 243$ & $1.381(5)$ \\
\hline $\mathrm{Si} 2-\mathrm{C} 212$ & $1.864(4)$ & $\mathrm{C} 243-\mathrm{C} 244$ & $1.377(8)$ \\
\hline $\mathrm{Si} 2-\mathrm{C} 211$ & $1.868(3)$ & $\mathrm{C} 244-\mathrm{C} 245$ & 1.353 \\
\hline
\end{tabular}




\begin{tabular}{|c|c|c|c|}
\hline $\mathrm{C} 245-\mathrm{C} 246$ & $1.384(7)$ & $\mathrm{C} 224-\mathrm{C} 225$ & $1.375(10)$ \\
\hline $\mathrm{C} 251-\mathrm{C} 256$ & $1.380(7)$ & $\mathrm{C} 225-\mathrm{C} 226$ & $1.402(8)$ \\
\hline $\mathrm{C} 251-\mathrm{C} 252$ & $1.381(7)$ & $\mathrm{C} 291-\mathrm{C} 296$ & $1.362(8)$ \\
\hline $\mathrm{C} 252-\mathrm{C} 253$ & $1.382(7)$ & $\mathrm{C} 291-\mathrm{C} 292$ & $1.373(8)$ \\
\hline $\mathrm{C} 253-\mathrm{C} 254$ & $1.381(19)$ & $\mathrm{C} 292-\mathrm{C} 293$ & $1.384(8)$ \\
\hline $\mathrm{C} 254-\mathrm{C} 255$ & $1.355(18)$ & $\mathrm{C} 293-\mathrm{C} 294$ & $1.370(16)$ \\
\hline $\mathrm{C} 255-\mathrm{C} 256$ & $1.386(11)$ & $\mathrm{C} 294-\mathrm{C} 295$ & $1.354(15)$ \\
\hline $\mathrm{C} 221-\mathrm{C} 226$ & $1.384(7)$ & $\mathrm{C} 295-\mathrm{C} 296$ & $1.390(13)$ \\
\hline $\mathrm{C} 221-\mathrm{C} 222$ & $1.419(6)$ & $\mathrm{C} 13 \mathrm{a}-\mathrm{C} 17 \mathrm{a}$ & $1.417(4)$ \\
\hline$C 222-\mathrm{C} 223$ & $1.382(7)$ & $\mathrm{C} 23 \mathrm{a}-\mathrm{C} 27 \mathrm{a}$ & $1.412(5)$ \\
\hline $\mathrm{C} 223-\mathrm{C} 224$ & $1.379(7)$ & & \\
\hline $\mathrm{C} 13-\mathrm{NI} 1-\mathrm{C} 12$ & $39.77(12)$ & $\mathrm{C} 131-\mathrm{P} 1-\mathrm{C} 161$ & $8.50(13)$ \\
\hline $\mathrm{C} 13-\mathrm{NI} 1-\mathrm{C} 11$ & $66.9(1)$ & $\mathrm{C} 121-\mathrm{P} 1-\mathrm{C} 161$ & $96.3(8)$ \\
\hline $\mathrm{C} 12-\mathrm{NI} 1-\mathrm{C} 11$ & $39.60(9)$ & $\mathrm{C} 141-\mathrm{P} 1-\mathrm{C} 161$ & $109.7(1)$ \\
\hline C13-NI1-CL1 & $162.96(8)$ & $\mathrm{C} 151-\mathrm{P} 1-\mathrm{NI} 1$ & $113.1(5)$ \\
\hline $\mathrm{C} 12-\mathrm{NI} 1-\mathrm{CL} 1$ & $124.76(8)$ & $\mathrm{C} 131-\mathrm{P} 1-\mathrm{NI} 1$ & $114.35(17)$ \\
\hline $\mathrm{C} 11-\mathrm{NI} 1-\mathrm{CL} 1$ & $96.23(7)$ & $\mathrm{C} 121-\mathrm{P} 1-\mathrm{NI} 1$ & $114.90(7)$ \\
\hline $\mathrm{C} 13-\mathrm{NI} 1-\mathrm{P} 1$ & $100.38(8)$ & $\mathrm{C} 141-\mathrm{P} 1-\mathrm{NI} 1$ & $114.98(15)$ \\
\hline $\mathrm{C} 12-\mathrm{NI} 1-\mathrm{P} 1$ & $131.84(7)$ & $\mathrm{C} 161-\mathrm{P} 1-\mathrm{NI} 1$ & $117.1(5)$ \\
\hline $\mathrm{C} 11-\mathrm{N} 11-\mathrm{P} 1$ & $166.65(7)$ & $\mathrm{C} 112-\mathrm{SI} 1-\mathrm{C} 113$ & $110.63(17)$ \\
\hline $\mathrm{CL} 1-\mathrm{NI} 1-\mathrm{P} 1$ & $96.61(3)$ & $\mathrm{C} 112-\mathrm{SI} 1-\mathrm{C} 111$ & $108.37(15)$ \\
\hline $\mathrm{C} 13-\mathrm{NI} 1-\mathrm{C} 13 \mathrm{~A}$ & $38.10(12)$ & $\mathrm{C} 113-\mathrm{S} 11-\mathrm{C} 111$ & $109.53(15)$ \\
\hline $\mathrm{C} 12-\mathrm{NI} 1-\mathrm{C} 13 \mathrm{~A}$ & $63.15(12)$ & $\mathrm{C} 112-\mathrm{SI} 1-\mathrm{C} 11$ & 113.38 \\
\hline $\mathrm{C} 11-\mathrm{NI} 1-\mathrm{C} 13 \mathrm{~A}$ & $63.32(9)$ & C113-SI1-C11 & 108.10 \\
\hline $\mathrm{CL} 1-\mathrm{NI} 1-\mathrm{C} 13 \mathrm{~A}$ & $137.25(9)$ & C111-SI1-C11 & $106.71(13)$ \\
\hline $\mathrm{P} 1-\mathrm{NI} 1-\mathrm{C} 13 \mathrm{~A}$ & $104.34(7)$ & $\mathrm{C} 12-\mathrm{C} 11-\mathrm{C} 17 \mathrm{~A}$ & $105.9(2)$ \\
\hline $\mathrm{C} 13-\mathrm{NI} 1-\mathrm{C} 17 \mathrm{~A}$ & $62.99(11)$ & $\mathrm{C} 12-\mathrm{C} 11-\mathrm{SI} 1$ & $123.72(19)$ \\
\hline $\mathrm{C} 12-\mathrm{NI} 1-\mathrm{C} 17 \mathrm{~A}$ & $62.88(10)$ & $\mathrm{C} 17 \mathrm{~A}-\mathrm{C} 11-\mathrm{SI} 1$ & $129.34(18)$ \\
\hline $\mathrm{C} 11-\mathrm{NI} 1-\mathrm{C} 17 \mathrm{~A}$ & $38.12(9)$ & $\mathrm{C} 12-\mathrm{C} 11-\mathrm{NI} 1$ & $68.01(13)$ \\
\hline $\mathrm{CL} 1-\mathrm{NI} 1-\mathrm{C} 17 \mathrm{~A}$ & $105.58(7)$ & $\mathrm{C} 17 \mathrm{~A}-\mathrm{C} 11-\mathrm{NI} 1$ & $78.60(13)$ \\
\hline $\mathrm{P} 1-\mathrm{NI} 1-\mathrm{C} 17 \mathrm{~A}$ & $133.40(6)$ & $\mathrm{SI} 1-\mathrm{C} 11-\mathrm{NI} 1$ & $126.85(12)$ \\
\hline $\mathrm{C} 13 \mathrm{~A}-\mathrm{NI} 1-\mathrm{C} 17 \mathrm{~A}$ & $35.41(9)$ & $\mathrm{C} 13-\mathrm{C} 12-\mathrm{C} 11$ & $109.4(3)$ \\
\hline $\mathrm{C} 151-\mathrm{P} 1-\mathrm{C} 131$ & $96.5(5)$ & $\mathrm{C} 13-\mathrm{C} 12-\mathrm{NI} 1$ & $69.44(15)$ \\
\hline $\mathrm{C} 151-\mathrm{P} 1-\mathrm{C} 121$ & $111.8(6)$ & $\mathrm{C} 11-\mathrm{C} 12-\mathrm{NI} 1$ & $72.39(14)$ \\
\hline $\mathrm{C} 131-\mathrm{P} 1-\mathrm{C} 121$ & $104.5(4)$ & $\mathrm{C} 12-\mathrm{C} 13-\mathrm{C} 13 \mathrm{~A}$ & $108.1(2)$ \\
\hline $\mathrm{C} 151-\mathrm{P} 1-\mathrm{C} 141$ & $12.0(7)$ & $\mathrm{C} 12-\mathrm{C} 13-\mathrm{NI} 1$ & $70.78(15)$ \\
\hline $\mathrm{C} 131-\mathrm{P} 1-\mathrm{C} 141$ & $105.4(4)$ & $\mathrm{C} 13 \mathrm{~A}-\mathrm{C} 13-\mathrm{NI} 1$ & $81.11(16)$ \\
\hline $\mathrm{C} 121-\mathrm{P} 1-\mathrm{C} 141$ & $101.28(18)$ & $\mathrm{C} 15-\mathrm{C} 14-\mathrm{C} 13 \mathrm{~A}$ & $118.2(3)$ \\
\hline $\mathrm{C} 151-\mathrm{P} 1-\mathrm{C} 161$ & $101.90(11)$ & $\mathrm{C} 14-\mathrm{C} 15-\mathrm{C} 16$ & $121.7(3)$ \\
\hline
\end{tabular}




\begin{tabular}{|c|c|}
\hline $\mathrm{C} 17-\mathrm{C} 16-\mathrm{C} 15$ & $120.8(4)$ \\
\hline $\mathrm{C} 16-\mathrm{C} 17-\mathrm{C} 17 \mathrm{~A}$ & $118.8(3)$ \\
\hline $\mathrm{C} 122-\mathrm{C} 121-\mathrm{C} 126$ & $117.7(2)$ \\
\hline $\mathrm{C} 122-\mathrm{C} 121-\mathrm{P} 1$ & $121.7(2)$ \\
\hline $\mathrm{C} 126-\mathrm{C} 121-\mathrm{P} 1$ & $120.25(18)$ \\
\hline $\mathrm{C} 121-\mathrm{C} 122-\mathrm{C} 123$ & $120.6(3)$ \\
\hline $\mathrm{C} 124-\mathrm{C} 123-\mathrm{C} 122$ & $120.7(3)$ \\
\hline $\mathrm{C} 123-\mathrm{C} 124-\mathrm{C} 125$ & $119.9(3)$ \\
\hline $\mathrm{C} 124-\mathrm{C} 125-\mathrm{C} 126$ & $119.7(3)$ \\
\hline $\mathrm{C} 125-\mathrm{C} 126-\mathrm{C} 121$ & $121.3(3)$ \\
\hline $\mathrm{C} 132-\mathrm{C} 131-\mathrm{C} 136$ & $121.9(4)$ \\
\hline $\mathrm{C} 132-\mathrm{C} 131-\mathrm{P} 1$ & $118.7(4)$ \\
\hline $\mathrm{C} 136-\mathrm{C} 131-\mathrm{P} 1$ & $119.3(4)$ \\
\hline $\mathrm{C} 131-\mathrm{C} 132-\mathrm{C} 133$ & $118.3(5)$ \\
\hline $\mathrm{C} 134-\mathrm{C} 133-\mathrm{C} 132$ & $120.7(6)$ \\
\hline $\mathrm{C} 135-\mathrm{C} 134-\mathrm{C} 133$ & $120.0(7)$ \\
\hline $\mathrm{C} 134-\mathrm{C} 135-\mathrm{C} 136$ & $120.9(6)$ \\
\hline $\mathrm{C} 131-\mathrm{C} 136-\mathrm{C} 135$ & $118.2(4)$ \\
\hline $\mathrm{C} 162-\mathrm{C} 161-\mathrm{C} 166$ & $106.7(1)$ \\
\hline $\mathrm{C} 162-\mathrm{C} 161-\mathrm{P} 1$ & $124.20(11)$ \\
\hline $\mathrm{C} 166-\mathrm{C} 161-\mathrm{P} 1$ & $128.9(1)$ \\
\hline $\mathrm{C} 163-\mathrm{C} 162-\mathrm{C} 161$ & $130.00(15)$ \\
\hline $\mathrm{C} 164-\mathrm{C} 163-\mathrm{C} 162$ & $118.10(18)$ \\
\hline $\mathrm{C} 163-\mathrm{C} 164-\mathrm{C} 165$ & $116.80(18)$ \\
\hline $\mathrm{C} 164-\mathrm{C} 165-\mathrm{C} 166$ & $120.20(13)$ \\
\hline $\mathrm{C} 161-\mathrm{C} 166-\mathrm{C} 165$ & $127.4(1)$ \\
\hline $\mathrm{C} 142-\mathrm{C} 141-\mathrm{C} 146$ & $116.6(3)$ \\
\hline $\mathrm{C} 142-\mathrm{C} 141-\mathrm{P} 1$ & $124.6(3)$ \\
\hline $\mathrm{C} 146-\mathrm{C} 141-\mathrm{P} 1$ & $118.7(3)$ \\
\hline $\mathrm{C} 141-\mathrm{C} 142-\mathrm{C} 143$ & $122.1(3)$ \\
\hline $\mathrm{C} 144-\mathrm{C} 143-\mathrm{C} 142$ & $120.2(5)$ \\
\hline $\mathrm{C} 143-\mathrm{C} 144-\mathrm{C} 145$ & $119.7(7)$ \\
\hline $\mathrm{C} 144-\mathrm{C} 145-\mathrm{C} 146$ & $119.8(6)$ \\
\hline $\mathrm{C} 141-\mathrm{C} 146-\mathrm{C} 145$ & $121.6(4)$ \\
\hline $\mathrm{C} 152-\mathrm{C} 151-\mathrm{C} 156$ & $128.4(9)$ \\
\hline $\mathrm{C} 152-\mathrm{C} 151-\mathrm{P} 1$ & $123.5(7)$ \\
\hline $\mathrm{C} 156-\mathrm{C} 151-\mathrm{P} 1$ & $107.6(7)$ \\
\hline $\mathrm{C} 151-\mathrm{C} 152-\mathrm{C} 153$ & $112.6(9)$ \\
\hline $\mathrm{C} 154-\mathrm{C} 153-\mathrm{C} 152$ & $122.40(12)$ \\
\hline
\end{tabular}

\begin{tabular}{|c|c|}
\hline $\mathrm{C} 153-\mathrm{C} 154-\mathrm{C} 155$ & $122.10(14)$ \\
\hline $\mathrm{C} 154-\mathrm{C} 155-\mathrm{C} 156$ & $118.20(11)$ \\
\hline $\mathrm{C} 151-\mathrm{C} 156-\mathrm{C} 155$ & $116.1(9)$ \\
\hline $\mathrm{C} 23-\mathrm{NI} 2-\mathrm{C} 22$ & $40.25(14)$ \\
\hline $\mathrm{C} 23-\mathrm{NI} 2-\mathrm{C} 21$ & $66.49(11)$ \\
\hline $\mathrm{C} 22-\mathrm{NI} 2-\mathrm{C} 21$ & $39.38(11)$ \\
\hline $\mathrm{C} 23-\mathrm{NI} 2-\mathrm{CL} 2 \mathrm{~A}$ & $163.87(11)$ \\
\hline $\mathrm{C} 22-\mathrm{NI} 2-\mathrm{CL} 2 \mathrm{~A}$ & $131.65(11)$ \\
\hline $\mathrm{C} 21-\mathrm{NI} 2-\mathrm{CL} 2 \mathrm{~A}$ & $99.32(9)$ \\
\hline $\mathrm{C} 23-\mathrm{NI} 2-\mathrm{P} 2$ & $99.14(9)$ \\
\hline $\mathrm{C} 22-\mathrm{NI} 2-\mathrm{P} 2$ & $127.33(9)$ \\
\hline $\mathrm{C} 21-\mathrm{NI} 2-\mathrm{P} 2$ & $165.40(8)$ \\
\hline $\mathrm{CL} 2 \mathrm{~A}-\mathrm{NI} 2-\mathrm{P} 2$ & $95.26(6)$ \\
\hline $\mathrm{C} 23-\mathrm{NI} 2-\mathrm{CL} 2 \mathrm{~B}$ & $160.74(17)$ \\
\hline $\mathrm{C} 22-\mathrm{NI} 2-\mathrm{CL} 2 \mathrm{~B}$ & $120.92(16)$ \\
\hline $\mathrm{C} 21-\mathrm{NI} 2-\mathrm{CL} 2 \mathrm{~B}$ & $95.72(17)$ \\
\hline $\mathrm{CL} 2 \mathrm{~A}-\mathrm{NI} 2-\mathrm{CL} 2 \mathrm{~B}$ & $15.97(10)$ \\
\hline $\mathrm{P} 2-\mathrm{NI} 2-\mathrm{CL} 2 \mathrm{~B}$ & $98.14(16)$ \\
\hline $\mathrm{C} 23-\mathrm{NI} 2-\mathrm{C} 23 \mathrm{~A}$ & $37.88(14)$ \\
\hline $\mathrm{C} 22-\mathrm{NI} 2-\mathrm{C} 23 \mathrm{~A}$ & $63.24(14)$ \\
\hline $\mathrm{C} 21-\mathrm{NI} 2-\mathrm{C} 23 \mathrm{~A}$ & $62.46(12)$ \\
\hline $\mathrm{CL} 2 \mathrm{~A}-\mathrm{NI} 2-\mathrm{C} 23 \mathrm{~A}$ & $129.62(11)$ \\
\hline $\mathrm{P} 2-\mathrm{NI} 2-\mathrm{C} 23 \mathrm{~A}$ & $108.18(9)$ \\
\hline $\mathrm{CL} 2 \mathrm{~B}-\mathrm{NI} 2-\mathrm{C} 23 \mathrm{~A}$ & $140.83(18)$ \\
\hline $\mathrm{C} 23-\mathrm{NI} 2-\mathrm{C} 27 \mathrm{~A}$ & $62.37(12)$ \\
\hline $\mathrm{C} 22-\mathrm{NI} 2-\mathrm{C} 27 \mathrm{~A}$ & $62.55(13)$ \\
\hline $\mathrm{C} 21-\mathrm{NI} 2-\mathrm{C} 27 \mathrm{~A}$ & $37.47(12)$ \\
\hline $\mathrm{CL} 2 \mathrm{~A}-\mathrm{NI} 2-\mathrm{C} 27 \mathrm{~A}$ & $101.80(9)$ \\
\hline $\mathrm{P} 2-\mathrm{NI} 2-\mathrm{C} 27 \mathrm{~A}$ & $139.11(9)$ \\
\hline $\mathrm{CL} 2 \mathrm{~B}-\mathrm{NI} 2-\mathrm{C} 27 \mathrm{~A}$ & $108.37(18)$ \\
\hline $\mathrm{C} 23 \mathrm{~A}-\mathrm{NI} 2-\mathrm{C} 27 \mathrm{~A}$ & $34.87(11)$ \\
\hline $\mathrm{CL} 2 \mathrm{~B}-\mathrm{CL} 2 \mathrm{~A}-\mathrm{NI} 2$ & $83.69(19)$ \\
\hline CL2A-CL2B-NI2 & $80.3(2)$ \\
\hline $\mathrm{C} 291-\mathrm{P} 2-\mathrm{C} 271$ & $92.1(6)$ \\
\hline $\mathrm{C} 291-\mathrm{P} 2-\mathrm{C} 231$ & $84.9(7)$ \\
\hline $\mathrm{C} 271-\mathrm{P} 2-\mathrm{C} 231$ & $9.2(8)$ \\
\hline $\mathrm{C} 291-\mathrm{P} 2-\mathrm{C} 241$ & $102.6(5)$ \\
\hline $\mathrm{C} 271-\mathrm{P} 2-\mathrm{C} 241$ & $104.9(6)$ \\
\hline $\mathrm{C} 231-\mathrm{P} 2-\mathrm{C} 241$ & $112.0(5)$ \\
\hline
\end{tabular}




\begin{tabular}{|c|c|c|c|}
\hline $\mathrm{C} 291-\mathrm{P} 2-\mathrm{C} 281$ & $104.3(9)$ & $\mathrm{C} 22-\mathrm{C} 21-\mathrm{NI} 2$ & $67.46(15)$ \\
\hline $\mathrm{C} 271-\mathrm{P} 2-\mathrm{C} 281$ & $13.1(6)$ & $\mathrm{C} 27 \mathrm{~A}-\mathrm{C} 21-\mathrm{NI} 2$ & $80.14(16)$ \\
\hline $\mathrm{C} 231-\mathrm{P} 2-\mathrm{C} 281$ & $22.1(1)$ & $\mathrm{SI} 2-\mathrm{C} 21-\mathrm{NI} 2$ & $127.32(15)$ \\
\hline $\mathrm{C} 241-\mathrm{P} 2-\mathrm{C} 281$ & $97.1(8)$ & $\mathrm{C} 23-\mathrm{C} 22-\mathrm{C} 21$ & $108.2(3)$ \\
\hline $\mathrm{C} 291-\mathrm{P} 2-\mathrm{C} 251$ & $110.5(8)$ & $\mathrm{C} 23-\mathrm{C} 22-\mathrm{NI} 2$ & $69.05(16)$ \\
\hline $\mathrm{C} 271-\mathrm{P} 2-\mathrm{C} 251$ & $103.8(6)$ & $\mathrm{C} 21-\mathrm{C} 22-\mathrm{NI} 2$ & $73.16(16)$ \\
\hline $\mathrm{C} 231-\mathrm{P} 2-\mathrm{C} 251$ & $111.7(6)$ & $\mathrm{C} 22-\mathrm{C} 23-\mathrm{C} 23 \mathrm{~A}$ & $108.4(3)$ \\
\hline $\mathrm{C} 241-\mathrm{P} 2-\mathrm{C} 251$ & $7.9(8)$ & $\mathrm{C} 22-\mathrm{C} 23-\mathrm{NI} 2$ & $70.70(17)$ \\
\hline $\mathrm{C} 281-\mathrm{P} 2-\mathrm{C} 251$ & $94.6(9)$ & $\mathrm{C} 23 \mathrm{~A}-\mathrm{C} 23-\mathrm{NI} 2$ & $82.40(18)$ \\
\hline $\mathrm{C} 291-\mathrm{P} 2-\mathrm{C} 261$ & $103.4(7)$ & $\mathrm{C} 25-\mathrm{C} 24-\mathrm{C} 23 \mathrm{~A}$ & $118.5(5)$ \\
\hline $\mathrm{C} 271-\mathrm{P} 2-\mathrm{C} 261$ & $12.0(8)$ & $\mathrm{C} 26-\mathrm{C} 25-\mathrm{C} 24$ & $122.1(5)$ \\
\hline $\mathrm{C} 231-\mathrm{P} 2-\mathrm{C} 261$ & $18.6(7)$ & $\mathrm{C} 25-\mathrm{C} 26-\mathrm{C} 27$ & $122.2(6)$ \\
\hline $\mathrm{C} 241-\mathrm{P} 2-\mathrm{C} 261$ & $106.0(9)$ & $\mathrm{C} 26-\mathrm{C} 27-\mathrm{C} 27 \mathrm{~A}$ & $118.4(5)$ \\
\hline $\mathrm{C} 281-\mathrm{P} 2-\mathrm{C} 261$ & $9.10(14)$ & $\mathrm{C} 236-\mathrm{C} 231-\mathrm{C} 232$ & $118.5(1)$ \\
\hline $\mathrm{C} 251-\mathrm{P} 2-\mathrm{C} 261$ & $103.3(1)$ & $\mathrm{C} 236-\mathrm{C} 231-\mathrm{P} 2$ & $119.9(9)$ \\
\hline $\mathrm{C} 291-\mathrm{P} 2-\mathrm{C} 221$ & $12.4(5)$ & $\mathrm{C} 232-\mathrm{C} 231-\mathrm{P} 2$ & $115.6(9)$ \\
\hline $\mathrm{C} 271-\mathrm{P} 2-\mathrm{C} 221$ & $104.5(5)$ & $\mathrm{C} 233-\mathrm{C} 232-\mathrm{C} 231$ & $120.0(1)$ \\
\hline $\mathrm{C} 231-\mathrm{P} 2-\mathrm{C} 221$ & $97.2(6)$ & $\mathrm{C} 232-\mathrm{C} 233-\mathrm{C} 234$ & $114.60(12)$ \\
\hline $\mathrm{C} 241-\mathrm{P} 2-\mathrm{C} 221$ & $98.8(4)$ & $\mathrm{C} 235-\mathrm{C} 234-\mathrm{C} 233$ & $123.30(14)$ \\
\hline $\mathrm{C} 281-\mathrm{P} 2-\mathrm{C} 221$ & $116.6(7)$ & $\mathrm{C} 234-\mathrm{C} 235-\mathrm{C} 236$ & $118.10(12)$ \\
\hline $\mathrm{C} 251-\mathrm{P} 2-\mathrm{C} 221$ & $106.6(7)$ & $\mathrm{C} 231-\mathrm{C} 236-\mathrm{C} 235$ & $115.8(1)$ \\
\hline $\mathrm{C} 261-\mathrm{P} 2-\mathrm{C} 221$ & $115.8(6)$ & $\mathrm{C} 262-\mathrm{C} 261-\mathrm{C} 266$ & $111.40(11)$ \\
\hline $\mathrm{C} 291-\mathrm{P} 2-\mathrm{NI} 2$ & $119.8(6)$ & $\mathrm{C} 262-\mathrm{C} 261-\mathrm{P} 2$ & $127.70(11)$ \\
\hline $\mathrm{C} 271-\mathrm{P} 2-\mathrm{NI} 2$ & $118.3(5)$ & $\mathrm{C} 266-\mathrm{C} 261-\mathrm{P} 2$ & $121.00(12)$ \\
\hline $\mathrm{C} 231-\mathrm{P} 2-\mathrm{NI} 2$ & $117.6(6)$ & $\mathrm{C} 263-\mathrm{C} 262-\mathrm{C} 261$ & $126.60(13)$ \\
\hline $\mathrm{C} 241-\mathrm{P} 2-\mathrm{NI} 2$ & $115.53(19)$ & $\mathrm{C} 264-\mathrm{C} 263-\mathrm{C} 262$ & $119.30(15)$ \\
\hline $\mathrm{C} 281-\mathrm{P} 2-\mathrm{NI} 2$ & $114.5(7)$ & $\mathrm{C} 263-\mathrm{C} 264-\mathrm{C} 265$ & $119.30(15)$ \\
\hline $\mathrm{C} 251-\mathrm{P} 2-\mathrm{NI} 2$ & $110.2(4)$ & $\mathrm{C} 264-\mathrm{C} 265-\mathrm{C} 266$ & $118.80(16)$ \\
\hline $\mathrm{C} 261-\mathrm{P} 2-\mathrm{NI} 2$ & $108.1(7)$ & $\mathrm{C} 261-\mathrm{C} 266-\mathrm{C} 265$ & $123.80(15)$ \\
\hline $\mathrm{C} 221-\mathrm{P} 2-\mathrm{NI} 2$ & $112.4(3)$ & $\mathrm{C} 272-\mathrm{C} 271-\mathrm{C} 276$ & $105.5(9)$ \\
\hline C213-SI2-C212 & $109.5(3)$ & $\mathrm{C} 272-\mathrm{C} 271-\mathrm{P} 2$ & $126.80(11)$ \\
\hline C213-SI2-C211 & $110.1(2)$ & $\mathrm{C} 276-\mathrm{C} 271-\mathrm{P} 2$ & $112.4(1)$ \\
\hline $\mathrm{C} 212-\mathrm{SI} 2-\mathrm{C} 211$ & $109.7(2)$ & $\mathrm{C} 271-\mathrm{C} 272-\mathrm{C} 273$ & $128.30(14)$ \\
\hline C213-SI2-C21 & $108.63(19)$ & $\mathrm{C} 274-\mathrm{C} 273-\mathrm{C} 272$ & $117.10(15)$ \\
\hline $\mathrm{C} 212-\mathrm{SI} 2-\mathrm{C} 21$ & $112.54(18)$ & $\mathrm{C} 273-\mathrm{C} 274-\mathrm{C} 275$ & $110.40(14)$ \\
\hline C211-SI2-C21 & $106.40(17)$ & $\mathrm{C} 274-\mathrm{C} 275-\mathrm{C} 276$ & $126.10(12)$ \\
\hline $\mathrm{C} 22-\mathrm{C} 21-\mathrm{C} 27 \mathrm{~A}$ & $107.1(3)$ & $\mathrm{C} 271-\mathrm{C} 276-\mathrm{C} 275$ & $115.8(1)$ \\
\hline $\mathrm{C} 22-\mathrm{C} 21-\mathrm{SI} 2$ & $124.9(3)$ & $\mathrm{C} 282-\mathrm{C} 281-\mathrm{C} 286$ & $103.20(15)$ \\
\hline $\mathrm{C} 27 \mathrm{~A}-\mathrm{C} 21-\mathrm{SI} 2$ & $126.8(2)$ & $\mathrm{C} 282-\mathrm{C} 281-\mathrm{P} 2$ & $133.10(14)$ \\
\hline
\end{tabular}




\begin{tabular}{|c|c|c|c|}
\hline $\mathrm{C} 286-\mathrm{C} 281-\mathrm{P} 2$ & $111.90(14)$ & $\mathrm{C} 296-\mathrm{C} 291-\mathrm{P} 2$ & $113.5(7)$ \\
\hline $\mathrm{C} 281-\mathrm{C} 282-\mathrm{C} 283$ & $124.10(14)$ & $\mathrm{C} 292-\mathrm{C} 291-\mathrm{P} 2$ & $112.9(8)$ \\
\hline $\mathrm{C} 284-\mathrm{C} 283-\mathrm{C} 282$ & $118.60(16)$ & $\mathrm{C} 291-\mathrm{C} 292-\mathrm{C} 293$ & $113.2(9)$ \\
\hline $\mathrm{C} 285-\mathrm{C} 284-\mathrm{C} 283$ & $111.60(15)$ & $\mathrm{C} 294-\mathrm{C} 293-\mathrm{C} 292$ & $120.70(11)$ \\
\hline $\mathrm{C} 284-\mathrm{C} 285-\mathrm{C} 286$ & $123.50(18)$ & $\mathrm{C} 295-\mathrm{C} 294-\mathrm{C} 293$ & $120.30(11)$ \\
\hline $\mathrm{C} 281-\mathrm{C} 286-\mathrm{C} 285$ & $112.70(14)$ & $\mathrm{C} 294-\mathrm{C} 295-\mathrm{C} 296$ & $123.60(11)$ \\
\hline $\mathrm{C} 246-\mathrm{C} 241-\mathrm{C} 242$ & $118.4(4)$ & $\mathrm{C} 291-\mathrm{C} 296-\mathrm{C} 295$ & $110.9(1)$ \\
\hline $\mathrm{C} 246-\mathrm{C} 241-\mathrm{P} 2$ & $125.5(5)$ & $\mathrm{C} 14-\mathrm{C} 13 \mathrm{~A}-\mathrm{C} 17 \mathrm{~A}$ & $120.7(3)$ \\
\hline $\mathrm{C} 242-\mathrm{C} 241-\mathrm{P} 2$ & $116.1(4)$ & $\mathrm{C} 14-\mathrm{C} 13 \mathrm{~A}-\mathrm{C} 13$ & $132.3(3)$ \\
\hline $\mathrm{C} 241-\mathrm{C} 242-\mathrm{C} 243$ & $120.2(4)$ & $\mathrm{C} 17 \mathrm{~A}-\mathrm{C} 13 \mathrm{~A}-\mathrm{C} 13$ & $107.0(2)$ \\
\hline $\mathrm{C} 244-\mathrm{C} 243-\mathrm{C} 242$ & $120.8(6)$ & $\mathrm{C} 14-\mathrm{C} 13 \mathrm{~A}-\mathrm{NI} 1$ & $130.35(19)$ \\
\hline $\mathrm{C} 245-\mathrm{C} 244-\mathrm{C} 243$ & $118.9(8)$ & $\mathrm{C} 17 \mathrm{~A}-\mathrm{C} 13 \mathrm{~A}-\mathrm{NI} 1$ & $72.75(14)$ \\
\hline $\mathrm{C} 244-\mathrm{C} 245-\mathrm{C} 246$ & $120.8(6)$ & $\mathrm{C} 13-\mathrm{C} 13 \mathrm{~A}-\mathrm{NI} 1$ & $60.79(14)$ \\
\hline $\mathrm{C} 241-\mathrm{C} 246-\mathrm{C} 245$ & $120.8(5)$ & $\mathrm{C} 17-\mathrm{C} 17 \mathrm{~A}-\mathrm{C} 13 \mathrm{~A}$ & $119.7(3)$ \\
\hline $\mathrm{C} 256-\mathrm{C} 251-\mathrm{C} 252$ & $118.1(7)$ & $\mathrm{C} 17-\mathrm{C} 17 \mathrm{~A}-\mathrm{C} 11$ & $131.8(2)$ \\
\hline $\mathrm{C} 256-\mathrm{C} 251-\mathrm{P} 2$ & $117.1(6)$ & $\mathrm{C} 13 \mathrm{~A}-\mathrm{C} 17 \mathrm{~A}-\mathrm{C} 11$ & $108.4(2)$ \\
\hline $\mathrm{C} 252-\mathrm{C} 251-\mathrm{P} 2$ & $123.9(6)$ & $\mathrm{C} 17-\mathrm{C} 17 \mathrm{~A}-\mathrm{NI} 1$ & $129.17(18)$ \\
\hline $\mathrm{C} 251-\mathrm{C} 252-\mathrm{C} 253$ & $121.1(8)$ & $\mathrm{C} 13 \mathrm{~A}-\mathrm{C} 17 \mathrm{~A}-\mathrm{NI} 1$ & $71.84(15)$ \\
\hline $\mathrm{C} 254-\mathrm{C} 253-\mathrm{C} 252$ & $119.5(1)$ & $\mathrm{C} 11-\mathrm{C} 17 \mathrm{~A}-\mathrm{NI} 1$ & $63.28(12)$ \\
\hline $\mathrm{C} 255-\mathrm{C} 254-\mathrm{C} 253$ & $120.10(12)$ & $\mathrm{C} 27 \mathrm{~A}-\mathrm{C} 23 \mathrm{~A}-\mathrm{C} 24$ & $118.8(4)$ \\
\hline $\mathrm{C} 254-\mathrm{C} 255-\mathrm{C} 256$ & $120.30(11)$ & $\mathrm{C} 27 \mathrm{~A}-\mathrm{C} 23 \mathrm{~A}-\mathrm{C} 23$ & $107.1(3)$ \\
\hline $\mathrm{C} 251-\mathrm{C} 256-\mathrm{C} 255$ & $120.8(8)$ & $\mathrm{C} 24-\mathrm{C} 23 \mathrm{~A}-\mathrm{C} 23$ & $134.0(4)$ \\
\hline $\mathrm{C} 226-\mathrm{C} 221-\mathrm{C} 222$ & $112.1(6)$ & $\mathrm{C} 27 \mathrm{~A}-\mathrm{C} 23 \mathrm{~A}-\mathrm{NI} 2$ & $73.89(17)$ \\
\hline $\mathrm{C} 226-\mathrm{C} 221-\mathrm{P} 2$ & $124.6(4)$ & $\mathrm{C} 24-\mathrm{C} 23 \mathrm{~A}-\mathrm{NI} 2$ & $130.7(2)$ \\
\hline $\mathrm{C} 222-\mathrm{C} 221-\mathrm{P} 2$ & $123.2(5)$ & $\mathrm{C} 23-\mathrm{C} 23 \mathrm{~A}-\mathrm{NI} 2$ & $59.72(16)$ \\
\hline $\mathrm{C} 223-\mathrm{C} 222-\mathrm{C} 221$ & $122.7(7)$ & $\mathrm{C} 27-\mathrm{C} 27 \mathrm{~A}-\mathrm{C} 23 \mathrm{~A}$ & $119.9(3)$ \\
\hline $\mathrm{C} 224-\mathrm{C} 223-\mathrm{C} 222$ & $121.8(9)$ & $\mathrm{C} 27-\mathrm{C} 27 \mathrm{~A}-\mathrm{C} 21$ & $132.2(3)$ \\
\hline $\mathrm{C} 225-\mathrm{C} 224-\mathrm{C} 223$ & $118.3(9)$ & $\mathrm{C} 23 \mathrm{~A}-\mathrm{C} 27 \mathrm{~A}-\mathrm{C} 21$ & $107.9(3)$ \\
\hline $\mathrm{C} 224-\mathrm{C} 225-\mathrm{C} 226$ & $118.2(8)$ & $\mathrm{C} 27-\mathrm{C} 27 \mathrm{~A}-\mathrm{NI} 2$ & $130.5(2)$ \\
\hline $\mathrm{C} 221-\mathrm{C} 226-\mathrm{C} 225$ & $126.6(7)$ & $\mathrm{C} 23 \mathrm{~A}-\mathrm{C} 27 \mathrm{~A}-\mathrm{NI} 2$ & $71.25(16)$ \\
\hline $\begin{array}{l}\mathrm{C} 296-\mathrm{C} 291-\mathrm{C} 292 \\
\text { C13-NI1-P1-C151 }\end{array}$ & $\begin{array}{r}129.6(1) \\
-119.6(6)\end{array}$ & $\begin{array}{l}\mathrm{C} 21-\mathrm{C} 27 \mathrm{~A}-\mathrm{NI} 2 \\
\mathrm{C} 11-\mathrm{NI} 1-\mathrm{P} 1-\mathrm{C} 131\end{array}$ & $\begin{array}{l}62.39(14) \\
114.1(5)\end{array}$ \\
\hline $\mathrm{C} 12-\mathrm{NI} 1-\mathrm{P} 1-\mathrm{C} 151$ & $-91.8(6)$ & $\mathrm{CL} 1-\mathrm{N} 11-\mathrm{P} 1-\mathrm{C} 131$ & $-49.9(4)$ \\
\hline $\mathrm{C} 11-\mathrm{NI} 1-\mathrm{P} 1-\mathrm{C} 151$ & $-136.8(6)$ & $\mathrm{C} 13 \mathrm{~A}-\mathrm{N} 11-\mathrm{P} 1-\mathrm{C} 131$ & $92.5(5)$ \\
\hline $\mathrm{CL} 1-\mathrm{NI} 1-\mathrm{P} 1-\mathrm{C} 151$ & $59.2(6)$ & $\mathrm{C} 17 \mathrm{~A}-\mathrm{NI} 1-\mathrm{P} 1-\mathrm{C} 131$ & $68.9(5)$ \\
\hline $\mathrm{C} 13 \mathrm{~A}-\mathrm{NI} 1-\mathrm{P} 1-\mathrm{C} 151$ & $-158.4(6)$ & $\mathrm{C} 13-\mathrm{N} 11-\mathrm{P} 1-\mathrm{C} 121$ & $10.45(14)$ \\
\hline $\mathrm{C} 17 \mathrm{~A}-\mathrm{NI} 1-\mathrm{P} 1-\mathrm{C} 151$ & $178.0(6)$ & $\mathrm{C} 12-\mathrm{NI} 1-\mathrm{P} 1-\mathrm{C} 121$ & $38.23(15)$ \\
\hline $\mathrm{C} 13-\mathrm{NI} 1-\mathrm{P} 1-\mathrm{C} 131$ & $131.3(5)$ & $\mathrm{C} 11-\mathrm{NI} 1-\mathrm{P} 1-\mathrm{C} 121$ & $-6.8(3)$ \\
\hline $\mathrm{C} 12-\mathrm{NI} 1-\mathrm{P} 1-\mathrm{C} 131$ & $159.1(5)$ & $\mathrm{CL} 1-\mathrm{NI} 1-\mathrm{P} 1-\mathrm{C} 121$ & $-170.82(9)$ \\
\hline
\end{tabular}




\begin{tabular}{|c|c|c|c|}
\hline $\mathrm{C} 13 \mathrm{~A}-\mathrm{NI} 1-\mathrm{P} 1-\mathrm{C} 121$ & $-28.39(13)$ & $\mathrm{C} 17 \mathrm{~A}-\mathrm{C} 11-\mathrm{C} 12-\mathrm{C} 13$ & $10.5(3)$ \\
\hline $\mathrm{C} 17 \mathrm{~A}-\mathrm{NI} 1-\mathrm{P} 1-\mathrm{C} 121$ & $-52.03(13)$ & $\mathrm{SI} 1-\mathrm{C} 11-\mathrm{C} 12-\mathrm{C} 13$ & $179.59(17)$ \\
\hline $\mathrm{C} 13-\mathrm{NI} 1-\mathrm{P} 1-\mathrm{C} 141$ & $-106.6(2)$ & $\mathrm{N} 11-\mathrm{C} 11-\mathrm{C} 12-\mathrm{C} 13$ & $-59.87(18)$ \\
\hline $\mathrm{C} 12-\mathrm{NI} 1-\mathrm{P} 1-\mathrm{C} 141$ & $-78.8(2)$ & $\mathrm{C} 17 \mathrm{~A}-\mathrm{C} 11-\mathrm{C} 12-\mathrm{NI} 1$ & $70.34(15)$ \\
\hline $\mathrm{C} 11-\mathrm{NI} 1-\mathrm{P} 1-\mathrm{C} 141$ & $-123.8(4)$ & $\mathrm{SI} 1-\mathrm{C} 11-\mathrm{C} 12-\mathrm{NI} 1$ & $-120.54(17)$ \\
\hline $\mathrm{CL} 1-\mathrm{NI} 1-\mathrm{P} 1-\mathrm{C} 141$ & $72.2(2)$ & $\mathrm{C} 11-\mathrm{NI} 1-\mathrm{C} 12-\mathrm{C} 13$ & $119.4(2)$ \\
\hline $\mathrm{C} 13 \mathrm{~A}-\mathrm{NI} 1-\mathrm{P} 1-\mathrm{C} 141$ & $-145.4(2)$ & $\mathrm{CL} 1-\mathrm{NI} 1-\mathrm{C} 12-\mathrm{C} 13$ & $170.18(15)$ \\
\hline $\mathrm{C} 17 \mathrm{~A}-\mathrm{NI} 1-\mathrm{P} 1-\mathrm{C} 141$ & $-169.0(2)$ & $\mathrm{P} 1-\mathrm{NI} 1-\mathrm{C} 12-\mathrm{C} 13$ & $-45.8(2)$ \\
\hline $\mathrm{C} 13-\mathrm{NI} 1-\mathrm{P} 1-\mathrm{C} 161$ & $122.40(12)$ & $\mathrm{C} 13 \mathrm{~A}-\mathrm{NI} 1-\mathrm{C} 12-\mathrm{C} 13$ & $39.60(17)$ \\
\hline $\mathrm{C} 12-\mathrm{NI} 1-\mathrm{P} 1-\mathrm{C} 161$ & $150.20(12)$ & $\mathrm{C} 17 \mathrm{~A}-\mathrm{NI} 1-\mathrm{C} 12-\mathrm{C} 13$ & $79.50(19)$ \\
\hline $\mathrm{C} 11-\mathrm{NI} 1-\mathrm{P} 1-\mathrm{C} 161$ & $105.10(13)$ & $\mathrm{C} 13-\mathrm{NI} 1-\mathrm{C} 12-\mathrm{C} 11$ & $-119.4(2)$ \\
\hline $\mathrm{CL} 1-\mathrm{NI} 1-\mathrm{P} 1-\mathrm{C} 161$ & $-58.90(12)$ & $\mathrm{CL} 1-\mathrm{NI} 1-\mathrm{C} 12-\mathrm{C} 11$ & $50.82(18)$ \\
\hline $\mathrm{C} 13 \mathrm{~A}-\mathrm{NI} 1-\mathrm{P} 1-\mathrm{C} 161$ & $83.60(12)$ & $\mathrm{P} 1-\mathrm{NI} 1-\mathrm{C} 12-\mathrm{C} 11$ & $-165.14(11)$ \\
\hline $\mathrm{C} 17 \mathrm{~A}-\mathrm{NI} 1-\mathrm{P} 1-\mathrm{C} 161$ & $59.90(12)$ & $\mathrm{C} 13 \mathrm{~A}-\mathrm{NI} 1-\mathrm{C} 12-\mathrm{C} 11$ & $-79.76(17)$ \\
\hline $\mathrm{C} 112-\mathrm{SI} 1-\mathrm{C} 11-\mathrm{C} 12$ & $159.4(2)$ & $\mathrm{C} 17 \mathrm{~A}-\mathrm{NI} 1-\mathrm{C} 12-\mathrm{C} 11$ & $-39.86(14)$ \\
\hline $\mathrm{C} 113-\mathrm{S} 11-\mathrm{C} 11-\mathrm{C} 12$ & $36.3(2)$ & $\mathrm{C} 11-\mathrm{C} 12-\mathrm{C} 13-\mathrm{C} 13 \mathrm{~A}$ & $-11.7(3)$ \\
\hline $\mathrm{C} 111-\mathrm{S} 11-\mathrm{C} 11-\mathrm{C} 12$ & $-81.4(2)$ & $\mathrm{NI} 1-\mathrm{C} 12-\mathrm{C} 13-\mathrm{C} 13 \mathrm{~A}$ & $-73.36(18)$ \\
\hline $\mathrm{C} 112-\mathrm{S} 11-\mathrm{C} 11-\mathrm{C} 17 \mathrm{~A}$ & $-34.2(3)$ & $\mathrm{C} 11-\mathrm{C} 12-\mathrm{C} 13-\mathrm{NI} 1$ & $61.70(18)$ \\
\hline $\mathrm{C} 113-\mathrm{SI} 1-\mathrm{C} 11-\mathrm{C} 17 \mathrm{~A}$ & $-157.2(2)$ & $\mathrm{C} 11-\mathrm{N} 11-\mathrm{C} 13-\mathrm{C} 12$ & $-37.15(16)$ \\
\hline $\mathrm{C} 111-\mathrm{S} 11-\mathrm{C} 11-\mathrm{C} 17 \mathrm{~A}$ & $85.0(2)$ & $\mathrm{CL} 1-\mathrm{NI} 1-\mathrm{C} 13-\mathrm{C} 12$ & $-28.6(5)$ \\
\hline $\mathrm{C} 112-\mathrm{SI} 1-\mathrm{C} 11-\mathrm{NI} 1$ & $72.9(2)$ & $\mathrm{P} 1-\mathrm{NI} 1-\mathrm{C} 13-\mathrm{C} 12$ & $147.13(16)$ \\
\hline C113-SI1-C11-NI1 & $-50.1(2)$ & $\mathrm{C} 13 \mathrm{~A}-\mathrm{NI} 1-\mathrm{C} 13-\mathrm{C} 12$ & $-112.8(2)$ \\
\hline $\mathrm{C} 111-\mathrm{SI} 1-\mathrm{C} 11-\mathrm{NI} 1$ & $-167.82(15)$ & $\mathrm{C} 17 \mathrm{~A}-\mathrm{NI} 1-\mathrm{C} 13-\mathrm{C} 12$ & $-79.20(17)$ \\
\hline $\mathrm{C} 13-\mathrm{NI} 1-\mathrm{C} 11-\mathrm{C} 12$ & $37.31(18)$ & $\mathrm{C} 12-\mathrm{NI} 1-\mathrm{C} 13-\mathrm{C} 13 \mathrm{~A}$ & $112.8(2)$ \\
\hline $\mathrm{CL} 1-\mathrm{NI} 1-\mathrm{C} 11-\mathrm{C} 12$ & $-140.17(15)$ & $\mathrm{C} 11-\mathrm{N} 11-\mathrm{C} 13-\mathrm{C} 13 \mathrm{~A}$ & $75.68(16)$ \\
\hline $\mathrm{P} 1-\mathrm{NI} 1-\mathrm{C} 11-\mathrm{C} 12$ & $55.8(4)$ & $\mathrm{CL} 1-\mathrm{NI} 1-\mathrm{C} 13-\mathrm{C} 13 \mathrm{~A}$ & $84.3(4)$ \\
\hline $\mathrm{C} 13 \mathrm{~A}-\mathrm{NI} 1-\mathrm{C} 11-\mathrm{C} 12$ & $79.32(18)$ & $\mathrm{P} 1-\mathrm{NI} 1-\mathrm{C} 13-\mathrm{C} 13 \mathrm{~A}$ & $-100.04(14)$ \\
\hline $\mathrm{C} 17 \mathrm{~A}-\mathrm{NI} 1-\mathrm{C} 11-\mathrm{C} 12$ & $112.5(2)$ & $\mathrm{C} 17 \mathrm{~A}-\mathrm{NI} 1-\mathrm{C} 13-\mathrm{C} 13 \mathrm{~A}$ & $33.64(14)$ \\
\hline $\mathrm{C} 13-\mathrm{NI} 1-\mathrm{C} 11-\mathrm{C} 17 \mathrm{~A}$ & $-75.16(17)$ & $\mathrm{C} 13 \mathrm{~A}-\mathrm{C} 14-\mathrm{C} 15-\mathrm{C} 16$ & $2.6(5)$ \\
\hline $\mathrm{C} 12-\mathrm{NI} 1-\mathrm{C} 11-\mathrm{C} 17 \mathrm{~A}$ & $-112.5(2)$ & $\mathrm{C} 14-\mathrm{C} 15-\mathrm{C} 16-\mathrm{C} 17$ & $-1.6(6)$ \\
\hline CL1-NI1-C11-C17A & $107.37(13)$ & $\mathrm{C} 15-\mathrm{C} 16-\mathrm{C} 17-\mathrm{C} 17 \mathrm{~A}$ & $-1.2(5)$ \\
\hline $\mathrm{P} 1-\mathrm{NI} 1-\mathrm{C} 11-\mathrm{C} 17 \mathrm{~A}$ & $-56.6(4)$ & $\mathrm{C} 151-\mathrm{P} 1-\mathrm{C} 121-\mathrm{C} 122$ & $-130.6(4)$ \\
\hline $\mathrm{C} 13 \mathrm{~A}-\mathrm{NI} 1-\mathrm{C} 11-\mathrm{C} 17 \mathrm{~A}$ & $-33.15(15)$ & $\mathrm{C} 131-\mathrm{P} 1-\mathrm{C} 121-\mathrm{C} 122$ & $-27.3(4)$ \\
\hline $\mathrm{C} 13-\mathrm{NI} 1-\mathrm{C} 11-\mathrm{SI} 1$ & $153.8(2)$ & $\mathrm{C} 141-\mathrm{P} 1-\mathrm{C} 121-\mathrm{C} 122$ & $-136.6(3)$ \\
\hline $\mathrm{C} 12-\mathrm{NI} 1-\mathrm{C} 11-\mathrm{SI} 1$ & $116.5(2)$ & $\mathrm{C} 161-\mathrm{P} 1-\mathrm{C} 121-\mathrm{C} 122$ & $-25.0(9)$ \\
\hline CL1-NI1-C11-SI1 & $-23.70(15)$ & $\mathrm{NI} 1-\mathrm{P} 1-\mathrm{C} 121-\mathrm{C} 122$ & $98.8(2)$ \\
\hline $\mathrm{P} 1-\mathrm{NI} 1-\mathrm{C} 11-\mathrm{SI}$ & $172.28(18)$ & $\mathrm{C} 151-\mathrm{P} 1-\mathrm{C} 121-\mathrm{C} 126$ & $56.1(4)$ \\
\hline $\mathrm{C} 13 \mathrm{~A}-\mathrm{NI} 1-\mathrm{C} 11-\mathrm{SI} 1$ & $-164.2(2)$ & $\mathrm{C} 131-\mathrm{P} 1-\mathrm{C} 121-\mathrm{C} 126$ & $159.3(3)$ \\
\hline $\mathrm{C} 17 \mathrm{~A}-\mathrm{NI} 1-\mathrm{C} 11-\mathrm{SI} 1$ & $-131.1(2)$ & $\mathrm{C} 141-\mathrm{P} 1-\mathrm{C} 121-\mathrm{C} 126$ & $50.0(3)$ \\
\hline
\end{tabular}




\begin{tabular}{|c|c|c|c|}
\hline $\mathrm{C} 161-\mathrm{P} 1-\mathrm{C} 121-\mathrm{C} 126$ & $161.7(9)$ & $\mathrm{P} 1-\mathrm{C} 161-\mathrm{C} 162-\mathrm{C} 163$ & $175(2)$ \\
\hline $\mathrm{NI} 1-\mathrm{P} 1-\mathrm{C} 121-\mathrm{C} 126$ & $-74.5(2)$ & $\mathrm{C} 161-\mathrm{C} 162-\mathrm{C} 163-\mathrm{C} 164$ & $7(4)$ \\
\hline $\mathrm{C} 126-\mathrm{C} 121-\mathrm{C} 122-\mathrm{C} 123$ & $-0.5(5)$ & $\mathrm{C} 162-\mathrm{C} 163-\mathrm{C} 164-\mathrm{C} 165$ & $-10(5)$ \\
\hline $\mathrm{P} 1-\mathrm{C} 121-\mathrm{C} 122-\mathrm{C} 123$ & $-174.0(3)$ & $\mathrm{C} 163-\mathrm{C} 164-\mathrm{C} 165-\mathrm{C} 166$ & $8(5)$ \\
\hline $\mathrm{C} 121-\mathrm{C} 122-\mathrm{C} 123-\mathrm{C} 124$ & $-0.9(6)$ & $\mathrm{C} 162-\mathrm{C} 161-\mathrm{C} 166-\mathrm{C} 165$ & $-2(4)$ \\
\hline $\mathrm{C} 122-\mathrm{C} 123-\mathrm{C} 124-\mathrm{C} 125$ & $1.8(6)$ & $\mathrm{P} 1-\mathrm{C} 161-\mathrm{C} 166-\mathrm{C} 165$ & $-176.60(18)$ \\
\hline $\mathrm{C} 123-\mathrm{C} 124-\mathrm{C} 125-\mathrm{C} 126$ & $-1.4(6)$ & $\mathrm{C} 164-\mathrm{C} 165-\mathrm{C} 166-\mathrm{C} 161$ & $-2(4)$ \\
\hline $\mathrm{C} 124-\mathrm{C} 125-\mathrm{C} 126-\mathrm{C} 121$ & $0.0(5)$ & $\mathrm{C} 151-\mathrm{P} 1-\mathrm{C} 141-\mathrm{C} 142$ & $-127(3)$ \\
\hline $\mathrm{C} 122-\mathrm{C} 121-\mathrm{C} 126-\mathrm{C} 125$ & $0.9(4)$ & $\mathrm{C} 131-\mathrm{P} 1-\mathrm{C} 141-\mathrm{C} 142$ & $-84.0(6)$ \\
\hline $\mathrm{P} 1-\mathrm{C} 121-\mathrm{C} 126-\mathrm{C} 125$ & $174.5(3)$ & $\mathrm{C} 121-\mathrm{P} 1-\mathrm{C} 141-\mathrm{C} 142$ & $24.7(5)$ \\
\hline $\mathrm{C} 151-\mathrm{P} 1-\mathrm{C} 131-\mathrm{C} 132$ & $-137.3(1)$ & $\mathrm{C} 161-\mathrm{P} 1-\mathrm{C} 141-\mathrm{C} 142$ & $-76.3(9)$ \\
\hline $\mathrm{C} 121-\mathrm{P} 1-\mathrm{C} 131-\mathrm{C} 132$ & $108.1(8)$ & $\mathrm{NI} 1-\mathrm{P} 1-\mathrm{C} 141-\mathrm{C} 142$ & $149.2(4)$ \\
\hline $\mathrm{C} 141-\mathrm{P} 1-\mathrm{C} 131-\mathrm{C} 132$ & $-145.6(8)$ & $\mathrm{C} 151-\mathrm{P} 1-\mathrm{C} 141-\mathrm{C} 146$ & $48(2)$ \\
\hline $\mathrm{C} 161-\mathrm{P} 1-\mathrm{C} 131-\mathrm{C} 132$ & $92(6)$ & $\mathrm{C} 131-\mathrm{P} 1-\mathrm{C} 141-\mathrm{C} 146$ & $91.5(5)$ \\
\hline $\mathrm{NI} 1-\mathrm{P} 1-\mathrm{C} 131-\mathrm{C} 132$ & $-18.3(1)$ & $\mathrm{C} 121-\mathrm{P} 1-\mathrm{C} 141-\mathrm{C} 146$ & $-159.8(4)$ \\
\hline $\mathrm{C} 151-\mathrm{P} 1-\mathrm{C} 131-\mathrm{C} 136$ & $46.50(11)$ & $\mathrm{C} 161-\mathrm{P} 1-\mathrm{C} 141-\mathrm{C} 146$ & $99.2(8)$ \\
\hline $\mathrm{C} 121-\mathrm{P} 1-\mathrm{C} 131-\mathrm{C} 136$ & $-68.0(1)$ & $\mathrm{NI} 1-\mathrm{P} 1-\mathrm{C} 141-\mathrm{C} 146$ & $-35.3(5)$ \\
\hline $\mathrm{C} 141-\mathrm{P} 1-\mathrm{C} 131-\mathrm{C} 136$ & $38.3(1)$ & $\mathrm{C} 146-\mathrm{C} 141-\mathrm{C} 142-\mathrm{C} 143$ & $-0.2(8)$ \\
\hline $\mathrm{C} 161-\mathrm{P} 1-\mathrm{C} 131-\mathrm{C} 136$ & $-84(5)$ & $\mathrm{P} 1-\mathrm{C} 141-\mathrm{C} 142-\mathrm{C} 143$ & $175.4(4)$ \\
\hline $\mathrm{NI} 1-\mathrm{P} 1-\mathrm{C} 131-\mathrm{C} 136$ & $165.5(7)$ & $\mathrm{C} 141-\mathrm{C} 142-\mathrm{C} 143-\mathrm{C} 144$ & $-0.2(8)$ \\
\hline $\mathrm{C} 136-\mathrm{C} 131-\mathrm{C} 132-\mathrm{C} 133$ & $3.90(15)$ & $\mathrm{C} 142-\mathrm{C} 143-\mathrm{C} 144-\mathrm{C} 145$ & $-0.30(13)$ \\
\hline $\mathrm{P} 1-\mathrm{C} 131-\mathrm{C} 132-\mathrm{C} 133$ & $-172.2(7)$ & $\mathrm{C} 143-\mathrm{C} 144-\mathrm{C} 145-\mathrm{C} 146$ & $1.30(14)$ \\
\hline $\mathrm{C} 131-\mathrm{C} 132-\mathrm{C} 133-\mathrm{C} 134$ & $-3.40(13)$ & $\mathrm{C} 142-\mathrm{C} 141-\mathrm{C} 146-\mathrm{C} 145$ & $1.1(8)$ \\
\hline $\mathrm{C} 132-\mathrm{C} 133-\mathrm{C} 134-\mathrm{C} 135$ & $1.80(16)$ & $\mathrm{P} 1-\mathrm{C} 141-\mathrm{C} 146-\mathrm{C} 145$ & $-174.7(5)$ \\
\hline $\mathrm{C} 133-\mathrm{C} 134-\mathrm{C} 135-\mathrm{C} 136$ & $-0.70(17)$ & $\mathrm{C} 144-\mathrm{C} 145-\mathrm{C} 146-\mathrm{C} 141$ & $-1.70(12)$ \\
\hline $\mathrm{C} 132-\mathrm{C} 131-\mathrm{C} 136-\mathrm{C} 135$ & $-2.80(15)$ & $\mathrm{C} 131-\mathrm{P} 1-\mathrm{C} 151-\mathrm{C} 152$ & $-24.40(17)$ \\
\hline $\mathrm{P} 1-\mathrm{C} 131-\mathrm{C} 136-\mathrm{C} 135$ & $173.2(7)$ & $\mathrm{C} 121-\mathrm{P} 1-\mathrm{C} 151-\mathrm{C} 152$ & $84.10(16)$ \\
\hline $\mathrm{C} 134-\mathrm{C} 135-\mathrm{C} 136-\mathrm{C} 131$ & $1.20(14)$ & $\mathrm{C} 141-\mathrm{P} 1-\mathrm{C} 151-\mathrm{C} 152$ & $114(4)$ \\
\hline $\mathrm{C} 151-\mathrm{P} 1-\mathrm{C} 161-\mathrm{C} 162$ & $-102(3)$ & $\mathrm{C} 161-\mathrm{P} 1-\mathrm{C} 151-\mathrm{C} 152$ & $-17.80(18)$ \\
\hline $\mathrm{C} 131-\mathrm{P} 1-\mathrm{C} 161-\mathrm{C} 162$ & $-52(4)$ & $\mathrm{NI} 1-\mathrm{P} 1-\mathrm{C} 151-\mathrm{C} 152$ & $-144.40(14)$ \\
\hline $\mathrm{C} 121-\mathrm{P} 1-\mathrm{C} 161-\mathrm{C} 162$ & $144(3)$ & $\mathrm{C} 131-\mathrm{P} 1-\mathrm{C} 151-\mathrm{C} 156$ & $162.60(12)$ \\
\hline $\mathrm{C} 141-\mathrm{P} 1-\mathrm{C} 161-\mathrm{C} 162$ & $-112(3)$ & $\mathrm{C} 121-\mathrm{P} 1-\mathrm{C} 151-\mathrm{C} 156$ & $-88.90(12)$ \\
\hline $\mathrm{NI} 1-\mathrm{P} 1-\mathrm{C} 161-\mathrm{C} 162$ & $22(3)$ & $\mathrm{C} 141-\mathrm{P} 1-\mathrm{C} 151-\mathrm{C} 156$ & $-59.20(19)$ \\
\hline $\mathrm{C} 151-\mathrm{P} 1-\mathrm{C} 161-\mathrm{C} 166$ & $72(3)$ & $\mathrm{C} 161-\mathrm{P} 1-\mathrm{C} 151-\mathrm{C} 156$ & $169.20(12)$ \\
\hline $\mathrm{C} 131-\mathrm{P} 1-\mathrm{C} 161-\mathrm{C} 166$ & $123(8)$ & $\mathrm{NI} 1-\mathrm{P} 1-\mathrm{C} 151-\mathrm{C} 156$ & $42.60(14)$ \\
\hline $\mathrm{C} 121-\mathrm{P} 1-\mathrm{C} 161-\mathrm{C} 166$ & $-42(3)$ & $\mathrm{C} 156-\mathrm{C} 151-\mathrm{C} 152-\mathrm{C} 153$ & $-1(3)$ \\
\hline $\mathrm{C} 141-\mathrm{P} 1-\mathrm{C} 161-\mathrm{C} 166$ & $62(3)$ & $\mathrm{P} 1-\mathrm{C} 151-\mathrm{C} 152-\mathrm{C} 153$ & $-172.00(15)$ \\
\hline $\mathrm{NI} 1-\mathrm{P} 1-\mathrm{C} 161-\mathrm{C} 166$ & $-164(2)$ & $\mathrm{C} 151-\mathrm{C} 152-\mathrm{C} 153-\mathrm{C} 154$ & $-2(3)$ \\
\hline $\mathrm{C} 166-\mathrm{C} 161-\mathrm{C} 162-\mathrm{C} 163$ & $-1(4)$ & $\mathrm{C} 152-\mathrm{C} 153-\mathrm{C} 154-\mathrm{C} 155$ & $3(5)$ \\
\hline
\end{tabular}




\begin{tabular}{|c|c|c|c|}
\hline $\mathrm{C} 153-\mathrm{C} 154-\mathrm{C} 155-\mathrm{C} 156$ & $-3(4)$ & $\mathrm{C} 21-\mathrm{NI} 2-\mathrm{P} 2-\mathrm{C} 241$ & $105.8(5)$ \\
\hline $\mathrm{C} 152-\mathrm{C} 151-\mathrm{C} 156-\mathrm{C} 155$ & $1(3)$ & $\mathrm{CL} 2 \mathrm{~A}-\mathrm{NI} 2-\mathrm{P} 2-\mathrm{C} 241$ & $-71.5(3)$ \\
\hline $\mathrm{P} 1-\mathrm{C} 151-\mathrm{C} 156-\mathrm{C} 155$ & $173.7(1)$ & $\mathrm{CL} 2 \mathrm{~B}-\mathrm{NI} 2-\mathrm{P} 2-\mathrm{C} 241$ & $-55.7(3)$ \\
\hline $\mathrm{C} 154-\mathrm{C} 155-\mathrm{C} 156-\mathrm{C} 151$ & $0(3)$ & $\mathrm{C} 23 \mathrm{~A}-\mathrm{NI} 2-\mathrm{P} 2-\mathrm{C} 241$ & $153.7(3)$ \\
\hline C23-NI2-CL2A-CL2B & $-105.6(8)$ & $\mathrm{C} 27 \mathrm{~A}-\mathrm{NI} 2-\mathrm{P} 2-\mathrm{C} 241$ & $173.7(3)$ \\
\hline $\mathrm{C} 22-\mathrm{NI} 2-\mathrm{CL} 2 \mathrm{~A}-\mathrm{CL} 2 \mathrm{~B}$ & $-52.5(8)$ & $\mathrm{C} 23-\mathrm{NI} 2-\mathrm{P} 2-\mathrm{C} 281$ & $-132.7(9)$ \\
\hline $\mathrm{C} 21-\mathrm{NI} 2-\mathrm{CL} 2 \mathrm{~A}-\mathrm{CL} 2 \mathrm{~B}$ & $-78.1(7)$ & $\mathrm{C} 22-\mathrm{NI} 2-\mathrm{P} 2-\mathrm{C} 281$ & $-164.5(9)$ \\
\hline $\mathrm{P} 2-\mathrm{NI} 2-\mathrm{CL} 2 \mathrm{~A}-\mathrm{CL} 2 \mathrm{~B}$ & $101.2(7)$ & $\mathrm{C} 21-\mathrm{NI} 2-\mathrm{P} 2-\mathrm{C} 281$ & $-142.7(9)$ \\
\hline $\mathrm{C} 23 \mathrm{~A}-\mathrm{NI} 2-\mathrm{CL} 2 \mathrm{~A}-\mathrm{CL} 2 \mathrm{~B}$ & $-139.9(7)$ & $\mathrm{CL} 2 \mathrm{~A}-\mathrm{NI} 2-\mathrm{P} 2-\mathrm{C} 281$ & $40.0(9)$ \\
\hline $\mathrm{C} 27 \mathrm{~A}-\mathrm{NI} 2-\mathrm{CL} 2 \mathrm{~A}-\mathrm{CL} 2 \mathrm{~B}$ & $-116.1(7)$ & $\mathrm{CL} 2 \mathrm{~B}-\mathrm{NI} 2-\mathrm{P} 2-\mathrm{C} 281$ & $55.9(9)$ \\
\hline $\mathrm{C} 23-\mathrm{NI} 2-\mathrm{CL} 2 \mathrm{~B}-\mathrm{CL} 2 \mathrm{~A}$ & $125.8(6)$ & $\mathrm{C} 23 \mathrm{~A}-\mathrm{NI} 2-\mathrm{P} 2-\mathrm{C} 281$ & $-94.7(9)$ \\
\hline $\mathrm{C} 22-\mathrm{NI} 2-\mathrm{CL} 2 \mathrm{~B}-\mathrm{CL} 2 \mathrm{~A}$ & $136.3(7)$ & $\mathrm{C} 27 \mathrm{~A}-\mathrm{NI} 2-\mathrm{P} 2-\mathrm{C} 281$ & $-74.8(9)$ \\
\hline $\mathrm{C} 21-\mathrm{NI} 2-\mathrm{CL} 2 \mathrm{~B}-\mathrm{CL} 2 \mathrm{~A}$ & $104.0(7)$ & $\mathrm{C} 23-\mathrm{NI} 2-\mathrm{P} 2-\mathrm{C} 251$ & $122.2(6)$ \\
\hline $\mathrm{P} 2-\mathrm{NI} 2-\mathrm{CL} 2 \mathrm{~B}-\mathrm{CL} 2 \mathrm{~A}$ & $-80.6(7)$ & $\mathrm{C} 22-\mathrm{NI} 2-\mathrm{P} 2-\mathrm{C} 251$ & $90.3(6)$ \\
\hline $\mathrm{C} 23 \mathrm{~A}-\mathrm{NI} 2-\mathrm{CL} 2 \mathrm{~B}-\mathrm{CL} 2 \mathrm{~A}$ & $51.8(9)$ & $\mathrm{C} 21-\mathrm{N} 12-\mathrm{P} 2-\mathrm{C} 251$ & $112.2(7)$ \\
\hline $\mathrm{C} 27 \mathrm{~A}-\mathrm{NI} 2-\mathrm{CL} 2 \mathrm{~B}-\mathrm{CL} 2 \mathrm{~A}$ & $67.8(7)$ & $\mathrm{CL} 2 \mathrm{~A}-\mathrm{NI} 2-\mathrm{P} 2-\mathrm{C} 251$ & $-65.1(6)$ \\
\hline $\mathrm{C} 23-\mathrm{NI} 2-\mathrm{P} 2-\mathrm{C} 291$ & $-7.7(4)$ & $\mathrm{CL} 2 \mathrm{~B}-\mathrm{NI} 2-\mathrm{P} 2-\mathrm{C} 251$ & $-49.3(6)$ \\
\hline $\mathrm{C} 22-\mathrm{NI} 2-\mathrm{P} 2-\mathrm{C} 291$ & $-39.6(4)$ & $\mathrm{C} 23 \mathrm{~A}-\mathrm{NI} 2-\mathrm{P} 2-\mathrm{C} 251$ & $160.1(6)$ \\
\hline $\mathrm{C} 21-\mathrm{NI} 2-\mathrm{P} 2-\mathrm{C} 291$ & $-17.7(5)$ & $\mathrm{C} 27 \mathrm{~A}-\mathrm{NI} 2-\mathrm{P} 2-\mathrm{C} 251$ & $-180.0(6)$ \\
\hline CL2A-NI2-P2-C291 & $165.0(4)$ & $\mathrm{C} 23-\mathrm{NI} 2-\mathrm{P} 2-\mathrm{C} 261$ & $-125.7(9)$ \\
\hline $\mathrm{CL} 2 \mathrm{~B}-\mathrm{NI} 2-\mathrm{P} 2-\mathrm{C} 291$ & $-179.2(4)$ & $\mathrm{C} 22-\mathrm{NI} 2-\mathrm{P} 2-\mathrm{C} 261$ & $-157.5(9)$ \\
\hline $\mathrm{C} 23 \mathrm{~A}-\mathrm{NI} 2-\mathrm{P} 2-\mathrm{C} 291$ & $30.2(4)$ & $\mathrm{C} 21-\mathrm{NI} 2-\mathrm{P} 2-\mathrm{C} 261$ & $-135.7(1)$ \\
\hline $\mathrm{C} 27 \mathrm{~A}-\mathrm{NI} 2-\mathrm{P} 2-\mathrm{C} 291$ & $50.2(4)$ & $\mathrm{CL} 2 \mathrm{~A}-\mathrm{NI} 2-\mathrm{P} 2-\mathrm{C} 261$ & $47.1(9)$ \\
\hline $\mathrm{C} 23-\mathrm{NI} 2-\mathrm{P} 2-\mathrm{C} 271$ & $-118.7(5)$ & $\mathrm{CL} 2 \mathrm{~B}-\mathrm{NI} 2-\mathrm{P} 2-\mathrm{C} 261$ & $62.9(9)$ \\
\hline $\mathrm{C} 22-\mathrm{NI} 2-\mathrm{P} 2-\mathrm{C} 271$ & $-150.5(5)$ & $\mathrm{C} 23 \mathrm{~A}-\mathrm{NI} 2-\mathrm{P} 2-\mathrm{C} 261$ & $-87.7(9)$ \\
\hline $\mathrm{C} 21-\mathrm{NI} 2-\mathrm{P} 2-\mathrm{C} 271$ & $-128.7(6)$ & $\mathrm{C} 27 \mathrm{~A}-\mathrm{NI} 2-\mathrm{P} 2-\mathrm{C} 261$ & $-67.8(9)$ \\
\hline CL2A-NI2-P2-C271 & $54.0(5)$ & $\mathrm{C} 23-\mathrm{NI} 2-\mathrm{P} 2-\mathrm{C} 221$ & $3.4(3)$ \\
\hline $\mathrm{CL} 2 \mathrm{~B}-\mathrm{NI} 2-\mathrm{P} 2-\mathrm{C} 271$ & $69.8(5)$ & $\mathrm{C} 22-\mathrm{NI} 2-\mathrm{P} 2-\mathrm{C} 221$ & $-28.5(3)$ \\
\hline $\mathrm{C} 23 \mathrm{~A}-\mathrm{NI} 2-\mathrm{P} 2-\mathrm{C} 271$ & $-80.8(5)$ & $\mathrm{C} 21-\mathrm{NI} 2-\mathrm{P} 2-\mathrm{C} 221$ & $-6.6(5)$ \\
\hline $\mathrm{C} 27 \mathrm{~A}-\mathrm{NI} 2-\mathrm{P} 2-\mathrm{C} 271$ & $-60.8(5)$ & $\mathrm{CL} 2 \mathrm{~A}-\mathrm{NI} 2-\mathrm{P} 2-\mathrm{C} 221$ & $176.1(2)$ \\
\hline $\mathrm{C} 23-\mathrm{NI} 2-\mathrm{P} 2-\mathrm{C} 231$ & $-108.3(6)$ & $\mathrm{CL} 2 \mathrm{~B}-\mathrm{NI} 2-\mathrm{P} 2-\mathrm{C} 221$ & $-168.1(3)$ \\
\hline $\mathrm{C} 22-\mathrm{NI} 2-\mathrm{P} 2-\mathrm{C} 231$ & $-140.1(6)$ & $\mathrm{C} 23 \mathrm{~A}-\mathrm{NI} 2-\mathrm{P} 2-\mathrm{C} 221$ & $41.3(3)$ \\
\hline $\mathrm{C} 21-\mathrm{NI} 2-\mathrm{P} 2-\mathrm{C} 231$ & $-118.3(7)$ & $\mathrm{C} 27 \mathrm{~A}-\mathrm{NI} 2-\mathrm{P} 2-\mathrm{C} 221$ & $61.2(3)$ \\
\hline $\mathrm{CL} 2 \mathrm{~A}-\mathrm{NI} 2-\mathrm{P} 2-\mathrm{C} 231$ & $64.4(5)$ & $\mathrm{C} 213-\mathrm{SI} 2-\mathrm{C} 21-\mathrm{C} 22$ & $-33.4(3)$ \\
\hline $\mathrm{CL} 2 \mathrm{~B}-\mathrm{NI} 2-\mathrm{P} 2-\mathrm{C} 231$ & $80.2(6)$ & $\mathrm{C} 212-\mathrm{SI} 2-\mathrm{C} 21-\mathrm{C} 22$ & $-154.8(3)$ \\
\hline $\mathrm{C} 23 \mathrm{~A}-\mathrm{NI} 2-\mathrm{P} 2-\mathrm{C} 231$ & $-70.4(5)$ & $\mathrm{C} 211-\mathrm{SI} 2-\mathrm{C} 21-\mathrm{C} 22$ & $85.1(3)$ \\
\hline $\mathrm{C} 27 \mathrm{~A}-\mathrm{NI} 2-\mathrm{P} 2-\mathrm{C} 231$ & $-50.4(6)$ & $\mathrm{C} 213-\mathrm{S} 2-\mathrm{C} 21-\mathrm{C} 27 \mathrm{~A}$ & $160.9(3)$ \\
\hline $\mathrm{C} 23-\mathrm{NI} 2-\mathrm{P} 2-\mathrm{C} 241$ & $115.8(3)$ & $\mathrm{C} 212-\mathrm{SI} 2-\mathrm{C} 21-\mathrm{C} 27 \mathrm{~A}$ & $39.5(3)$ \\
\hline $\mathrm{C} 22-\mathrm{NI} 2-\mathrm{P} 2-\mathrm{C} 241$ & $83.9(3)$ & $\mathrm{C} 211-\mathrm{SI} 2-\mathrm{C} 21-\mathrm{C} 27 \mathrm{~A}$ & $-80.6(3)$ \\
\hline
\end{tabular}




\begin{tabular}{|c|c|c|c|}
\hline $\mathrm{C} 213-\mathrm{SI} 2-\mathrm{C} 21-\mathrm{NI} 2$ & $53.4(3)$ & $\mathrm{C} 21-\mathrm{C} 22-\mathrm{C} 23-\mathrm{C} 23 \mathrm{~A}$ & $11.6(3)$ \\
\hline $\mathrm{C} 212-\mathrm{SI} 2-\mathrm{C} 21-\mathrm{NI} 2$ & $-68.0(3)$ & $\mathrm{NI} 2-\mathrm{C} 22-\mathrm{C} 23-\mathrm{C} 23 \mathrm{~A}$ & $74.7(2)$ \\
\hline $\mathrm{C} 211-\mathrm{SI} 2-\mathrm{C} 21-\mathrm{NI} 2$ & $171.8(2)$ & $\mathrm{C} 21-\mathrm{C} 22-\mathrm{C} 23-\mathrm{NI} 2$ & $-63.1(2)$ \\
\hline $\mathrm{C} 23-\mathrm{NI} 2-\mathrm{C} 21-\mathrm{C} 22$ & $-38.6(2)$ & $\mathrm{C} 21-\mathrm{NI} 2-\mathrm{C} 23-\mathrm{C} 22$ & $37.75(19)$ \\
\hline $\mathrm{CL} 2 \mathrm{~A}-\mathrm{NI} 2-\mathrm{C} 21-\mathrm{C} 22$ & $149.5(2)$ & $\mathrm{CL} 2 \mathrm{~A}-\mathrm{NI} 2-\mathrm{C} 23-\mathrm{C} 22$ & $67.5(5)$ \\
\hline $\mathrm{P} 2-\mathrm{NI} 2-\mathrm{C} 21-\mathrm{C} 22$ & $-27.8(5)$ & $\mathrm{P} 2-\mathrm{NI} 2-\mathrm{C} 23-\mathrm{C} 22$ & $-139.52(19)$ \\
\hline $\mathrm{CL} 2 \mathrm{~B}-\mathrm{NI} 2-\mathrm{C} 21-\mathrm{C} 22$ & $133.8(2)$ & $\mathrm{CL} 2 \mathrm{~B}-\mathrm{NI} 2-\mathrm{C} 23-\mathrm{C} 22$ & $14.0(6)$ \\
\hline $\mathrm{C} 23 \mathrm{~A}-\mathrm{NI} 2-\mathrm{C} 21-\mathrm{C} 22$ & $-80.5(2)$ & $\mathrm{C} 23 \mathrm{~A}-\mathrm{NI} 2-\mathrm{C} 23-\mathrm{C} 22$ & $112.6(3)$ \\
\hline $\mathrm{C} 27 \mathrm{~A}-\mathrm{NI} 2-\mathrm{C} 21-\mathrm{C} 22$ & $-113.3(3)$ & $\mathrm{C} 27 \mathrm{~A}-\mathrm{NI} 2-\mathrm{C} 23-\mathrm{C} 22$ & $79.2(2)$ \\
\hline $\mathrm{C} 23-\mathrm{NI} 2-\mathrm{C} 21-\mathrm{C} 27 \mathrm{~A}$ & $74.7(2)$ & $\mathrm{C} 22-\mathrm{NI} 2-\mathrm{C} 23-\mathrm{C} 23 \mathrm{~A}$ & $-112.6(3)$ \\
\hline $\mathrm{C} 22-\mathrm{NI} 2-\mathrm{C} 21-\mathrm{C} 27 \mathrm{~A}$ & $113.3(3)$ & $\mathrm{C} 21-\mathrm{NI} 2-\mathrm{C} 23-\mathrm{C} 23 \mathrm{~A}$ & $-74.8(2)$ \\
\hline $\mathrm{CL} 2 \mathrm{~A}-\mathrm{NI} 2-\mathrm{C} 21-\mathrm{C} 27 \mathrm{~A}$ & $-97.26(16)$ & $\mathrm{CL} 2 \mathrm{~A}-\mathrm{NI} 2-\mathrm{C} 23-\mathrm{C} 23 \mathrm{~A}$ & $-45.0(5)$ \\
\hline $\mathrm{P} 2-\mathrm{NI} 2-\mathrm{C} 21-\mathrm{C} 27 \mathrm{~A}$ & $85.5(4)$ & $\mathrm{P} 2-\mathrm{NI} 2-\mathrm{C} 23-\mathrm{C} 23 \mathrm{~A}$ & $107.92(17)$ \\
\hline $\mathrm{CL} 2 \mathrm{~B}-\mathrm{NI} 2-\mathrm{C} 21-\mathrm{C} 27 \mathrm{~A}$ & $-112.96(19)$ & $\mathrm{CL} 2 \mathrm{~B}-\mathrm{NI} 2-\mathrm{C} 23-\mathrm{C} 23 \mathrm{~A}$ & $-98.5(6)$ \\
\hline $\mathrm{C} 23 \mathrm{~A}-\mathrm{NI} 2-\mathrm{C} 21-\mathrm{C} 27 \mathrm{~A}$ & $32.77(17)$ & $\mathrm{C} 27 \mathrm{~A}-\mathrm{NI} 2-\mathrm{C} 23-\mathrm{C} 23 \mathrm{~A}$ & $-33.34(18)$ \\
\hline $\mathrm{C} 23-\mathrm{NI} 2-\mathrm{C} 21-\mathrm{SI} 2$ & $-156.1(3)$ & $\mathrm{C} 23 \mathrm{~A}-\mathrm{C} 24-\mathrm{C} 25-\mathrm{C} 26$ & $-2.3(8)$ \\
\hline $\mathrm{C} 22-\mathrm{NI} 2-\mathrm{C} 21-\mathrm{SI} 2$ & $-117.5(3)$ & $\mathrm{C} 24-\mathrm{C} 25-\mathrm{C} 26-\mathrm{C} 27$ & $1.1(9)$ \\
\hline $\mathrm{CL} 2 \mathrm{~A}-\mathrm{NI} 2-\mathrm{C} 21-\mathrm{SI} 2$ & $32.0(2)$ & $\mathrm{C} 25-\mathrm{C} 26-\mathrm{C} 27-\mathrm{C} 27 \mathrm{~A}$ & $1.4(8)$ \\
\hline $\mathrm{P} 2-\mathrm{NI} 2-\mathrm{C} 21-\mathrm{SI} 2$ & $-145.3(2)$ & $\mathrm{C} 291-\mathrm{P} 2-\mathrm{C} 231-\mathrm{C} 236$ & $95.70(14)$ \\
\hline $\mathrm{CL} 2 \mathrm{~B}-\mathrm{NI} 2-\mathrm{C} 21-\mathrm{SI} 2$ & $16.3(2)$ & $\mathrm{C} 271-\mathrm{P} 2-\mathrm{C} 231-\mathrm{C} 236$ & $-47(6)$ \\
\hline $\mathrm{C} 23 \mathrm{~A}-\mathrm{NI} 2-\mathrm{C} 21-\mathrm{SI} 2$ & $162.0(3)$ & $\mathrm{C} 241-\mathrm{P} 2-\mathrm{C} 231-\mathrm{C} 236$ & $-5.80(15)$ \\
\hline $\mathrm{C} 27 \mathrm{~A}-\mathrm{NI} 2-\mathrm{C} 21-\mathrm{SI} 2$ & $129.2(3)$ & $\mathrm{C} 281-\mathrm{P} 2-\mathrm{C} 231-\mathrm{C} 236$ & $-56(3)$ \\
\hline $\mathrm{C} 27 \mathrm{~A}-\mathrm{C} 21-\mathrm{C} 22-\mathrm{C} 23$ & $-10.8(3)$ & $\mathrm{C} 251-\mathrm{P} 2-\mathrm{C} 231-\mathrm{C} 236$ & $-14.40(17)$ \\
\hline $\mathrm{SI} 2-\mathrm{C} 21-\mathrm{C} 22-\mathrm{C} 23$ & $-178.8(2)$ & $\mathrm{C} 261-\mathrm{P} 2-\mathrm{C} 231-\mathrm{C} 236$ & $-80(3)$ \\
\hline $\mathrm{NI} 2-\mathrm{C} 21-\mathrm{C} 22-\mathrm{C} 23$ & $60.45(19)$ & $\mathrm{C} 221-\mathrm{P} 2-\mathrm{C} 231-\mathrm{C} 236$ & $96.80(14)$ \\
\hline $\mathrm{C} 27 \mathrm{~A}-\mathrm{C} 21-\mathrm{C} 22-\mathrm{NI} 2$ & $-71.25(18)$ & $\mathrm{NI} 2-\mathrm{P} 2-\mathrm{C} 231-\mathrm{C} 236$ & $-143.20(12)$ \\
\hline $\mathrm{SI} 2-\mathrm{C} 21-\mathrm{C} 22-\mathrm{NI} 2$ & $120.7(2)$ & $\mathrm{C} 291-\mathrm{P} 2-\mathrm{C} 231-\mathrm{C} 232$ & $-111.90(13)$ \\
\hline $\mathrm{C} 21-\mathrm{NI} 2-\mathrm{C} 22-\mathrm{C} 23$ & $-117.8(3)$ & $\mathrm{C} 271-\mathrm{P} 2-\mathrm{C} 231-\mathrm{C} 232$ & $106(7)$ \\
\hline $\mathrm{CL} 2 \mathrm{~A}-\mathrm{NI} 2-\mathrm{C} 22-\mathrm{C} 23$ & $-159.91(18)$ & $\mathrm{C} 241-\mathrm{P} 2-\mathrm{C} 231-\mathrm{C} 232$ & $146.60(11)$ \\
\hline $\mathrm{P} 2-\mathrm{NI} 2-\mathrm{C} 22-\mathrm{C} 23$ & $53.7(2)$ & $\mathrm{C} 281-\mathrm{P} 2-\mathrm{C} 231-\mathrm{C} 232$ & $96(3)$ \\
\hline $\mathrm{CL} 2 \mathrm{~B}-\mathrm{NI} 2-\mathrm{C} 22-\mathrm{C} 23$ & $-174.7(2)$ & $\mathrm{C} 251-\mathrm{P} 2-\mathrm{C} 231-\mathrm{C} 232$ & $138.00(12)$ \\
\hline $\mathrm{C} 23 \mathrm{~A}-\mathrm{NI} 2-\mathrm{C} 22-\mathrm{C} 23$ & $-39.4(2)$ & $\mathrm{C} 261-\mathrm{P} 2-\mathrm{C} 231-\mathrm{C} 232$ & $72(3)$ \\
\hline $\mathrm{C} 27 \mathrm{~A}-\mathrm{NI} 2-\mathrm{C} 22-\mathrm{C} 23$ & $-78.7(2)$ & $\mathrm{C} 221-\mathrm{P} 2-\mathrm{C} 231-\mathrm{C} 232$ & $-110.80(12)$ \\
\hline $\mathrm{C} 23-\mathrm{NI} 2-\mathrm{C} 22-\mathrm{C} 21$ & $117.8(3)$ & $\mathrm{NI} 2-\mathrm{P} 2-\mathrm{C} 231-\mathrm{C} 232$ & $9.20(14)$ \\
\hline $\mathrm{CL} 2 \mathrm{~A}-\mathrm{NI} 2-\mathrm{C} 22-\mathrm{C} 21$ & $-42.1(3)$ & $\mathrm{C} 236-\mathrm{C} 231-\mathrm{C} 232-\mathrm{C} 233$ & $-30(2)$ \\
\hline $\mathrm{P} 2-\mathrm{NI} 2-\mathrm{C} 22-\mathrm{C} 21$ & $171.50(15)$ & $\mathrm{P} 2-\mathrm{C} 231-\mathrm{C} 232-\mathrm{C} 233$ & $177.10(13)$ \\
\hline $\mathrm{CL} 2 \mathrm{~B}-\mathrm{NI} 2-\mathrm{C} 22-\mathrm{C} 21$ & $-56.9(3)$ & $\mathrm{C} 231-\mathrm{C} 232-\mathrm{C} 233-\mathrm{C} 234$ & $6(3)$ \\
\hline $\mathrm{C} 23 \mathrm{~A}-\mathrm{NI} 2-\mathrm{C} 22-\mathrm{C} 21$ & $78.4(2)$ & $\mathrm{C} 232-\mathrm{C} 233-\mathrm{C} 234-\mathrm{C} 235$ & $20(3)$ \\
\hline $\mathrm{C} 27 \mathrm{~A}-\mathrm{NI} 2-\mathrm{C} 22-\mathrm{C} 21$ & $39.03(19)$ & $\mathrm{C} 233-\mathrm{C} 234-\mathrm{C} 235-\mathrm{C} 236$ & $-21(4)$ \\
\hline
\end{tabular}




\begin{tabular}{|c|c|c|c|}
\hline $\mathrm{C} 232-\mathrm{C} 231-\mathrm{C} 236-\mathrm{C} 235$ & $28(2)$ & $\mathrm{C} 251-\mathrm{P} 2-\mathrm{C} 271-\mathrm{C} 276$ & $167.30(11)$ \\
\hline $\mathrm{P} 2-\mathrm{C} 231-\mathrm{C} 236-\mathrm{C} 235$ & $179.80(14)$ & $\mathrm{C} 261-\mathrm{P} 2-\mathrm{C} 271-\mathrm{C} 276$ & $78(7)$ \\
\hline $\mathrm{C} 234-\mathrm{C} 235-\mathrm{C} 236-\mathrm{C} 231$ & $-4(3)$ & $\mathrm{C} 221-\mathrm{P} 2-\mathrm{C} 271-\mathrm{C} 276$ & $-81.1(1)$ \\
\hline $\mathrm{C} 291-\mathrm{P} 2-\mathrm{C} 261-\mathrm{C} 262$ & $-152(2)$ & $\mathrm{NI} 2-\mathrm{P} 2-\mathrm{C} 271-\mathrm{C} 276$ & $44.90(11)$ \\
\hline $\mathrm{C} 271-\mathrm{P} 2-\mathrm{C} 261-\mathrm{C} 262$ & $-174(9)$ & $\mathrm{C} 276-\mathrm{C} 271-\mathrm{C} 272-\mathrm{C} 273$ & $-42(2)$ \\
\hline $\mathrm{C} 231-\mathrm{P} 2-\mathrm{C} 261-\mathrm{C} 262$ & $-148(5)$ & $\mathrm{P} 2-\mathrm{C} 271-\mathrm{C} 272-\mathrm{C} 273$ & $-176.10(16)$ \\
\hline $\mathrm{C} 241-\mathrm{P} 2-\mathrm{C} 261-\mathrm{C} 262$ & $100(2)$ & $\mathrm{C} 271-\mathrm{C} 272-\mathrm{C} 273-\mathrm{C} 274$ & $14(3)$ \\
\hline $\mathrm{C} 281-\mathrm{P} 2-\mathrm{C} 261-\mathrm{C} 262$ & $111(9)$ & $\mathrm{C} 272-\mathrm{C} 273-\mathrm{C} 274-\mathrm{C} 275$ & $21(3)$ \\
\hline $\mathrm{C} 251-\mathrm{P} 2-\mathrm{C} 261-\mathrm{C} 262$ & $93(3)$ & $\mathrm{C} 273-\mathrm{C} 274-\mathrm{C} 275-\mathrm{C} 276$ & $-27(4)$ \\
\hline $\mathrm{C} 221-\mathrm{P} 2-\mathrm{C} 261-\mathrm{C} 262$ & $-151(2)$ & $\mathrm{C} 272-\mathrm{C} 271-\mathrm{C} 276-\mathrm{C} 275$ & $32.30(18)$ \\
\hline $\mathrm{NI} 2-\mathrm{P} 2-\mathrm{C} 261-\mathrm{C} 262$ & $-24(3)$ & $\mathrm{P} 2-\mathrm{C} 271-\mathrm{C} 276-\mathrm{C} 275$ & $174.20(12)$ \\
\hline $\mathrm{C} 291-\mathrm{P} 2-\mathrm{C} 261-\mathrm{C} 266$ & $28(2)$ & $\mathrm{C} 274-\mathrm{C} 275-\mathrm{C} 276-\mathrm{C} 271$ & $-2(3)$ \\
\hline $\mathrm{C} 271-\mathrm{P} 2-\mathrm{C} 261-\mathrm{C} 266$ & $7(5)$ & $\mathrm{C} 291-\mathrm{P} 2-\mathrm{C} 281-\mathrm{C} 282$ & $-170(2)$ \\
\hline $\mathrm{C} 231-\mathrm{P} 2-\mathrm{C} 261-\mathrm{C} 266$ & $32.40(18)$ & $\mathrm{C} 271-\mathrm{P} 2-\mathrm{C} 281-\mathrm{C} 282$ & $-147(5)$ \\
\hline $241-\mathrm{P} 2-\mathrm{C} 261-\mathrm{C} 266$ & $-79(2)$ & $\mathrm{C} 231-\mathrm{P} 2-\mathrm{C} 281-\mathrm{C} 282$ & $-140(5)$ \\
\hline $281-\mathrm{P} 2-\mathrm{C} 261-\mathrm{C} 266$ & $-68(6)$ & $\mathrm{C} 241-\mathrm{P} 2-\mathrm{C} 281-\mathrm{C} 282$ & $85(2)$ \\
\hline $\mathrm{C} 251-\mathrm{P} 2-\mathrm{C} 261-\mathrm{C} 266$ & $-87(2)$ & $\mathrm{C} 251-\mathrm{P} 2-\mathrm{C} 281-\mathrm{C} 282$ & $78(2)$ \\
\hline $\mathrm{C} 221-\mathrm{P} 2-\mathrm{C} 261-\mathrm{C} 266$ & $29(3)$ & $\mathrm{C} 261-\mathrm{P} 2-\mathrm{C} 281-\mathrm{C} 282$ & $-84(7)$ \\
\hline $\mathrm{NI} 2-\mathrm{P} 2-\mathrm{C} 261-\mathrm{C} 266$ & $156(2)$ & $\mathrm{C} 221-\mathrm{P} 2-\mathrm{C} 281-\mathrm{C} 282$ & $-171(2)$ \\
\hline $266-\mathrm{C} 261-\mathrm{C} 262-\mathrm{C} 263$ & $-1(4)$ & $\mathrm{NI} 2-\mathrm{P} 2-\mathrm{C} 281-\mathrm{C} 282$ & $-37(3)$ \\
\hline $2-\mathrm{C} 261-\mathrm{C} 262-\mathrm{C} 263$ & $180(2)$ & $\mathrm{C} 291-\mathrm{P} 2-\mathrm{C} 281-\mathrm{C} 286$ & $55(2)$ \\
\hline 261-C262-C263-C264 & $2(4)$ & $\mathrm{C} 271-\mathrm{P} 2-\mathrm{C} 281-\mathrm{C} 286$ & $78(4)$ \\
\hline $\mathrm{C} 262-\mathrm{C} 263-\mathrm{C} 264-\mathrm{C} 265$ & $-7(4)$ & $\mathrm{C} 231-\mathrm{P} 2-\mathrm{C} 281-\mathrm{C} 286$ & $84(3)$ \\
\hline $\mathrm{C} 263-\mathrm{C} 264-\mathrm{C} 265-\mathrm{C} 266$ & $10(4)$ & $\mathrm{C} 241-\mathrm{P} 2-\mathrm{C} 281-\mathrm{C} 286$ & $-50.10(18)$ \\
\hline $\mathrm{C} 262-\mathrm{C} 261-\mathrm{C} 266-\mathrm{C} 265$ & $4(4)$ & $\mathrm{C} 251-\mathrm{P} 2-\mathrm{C} 281-\mathrm{C} 286$ & $-57.70(19)$ \\
\hline $\mathrm{P} 2-\mathrm{C} 261-\mathrm{C} 266-\mathrm{C} 265$ & $-176(2)$ & $\mathrm{C} 261-\mathrm{P} 2-\mathrm{C} 281-\mathrm{C} 286$ & $141(8)$ \\
\hline $264-\mathrm{C} 265-\mathrm{C} 266-\mathrm{C} 261$ & $-9(4)$ & $\mathrm{C} 221-\mathrm{P} 2-\mathrm{C} 281-\mathrm{C} 286$ & $54(2)$ \\
\hline $291-\mathrm{P} 2-\mathrm{C} 271-\mathrm{C} 272$ & $51.00(14)$ & $\mathrm{NI} 2-\mathrm{P} 2-\mathrm{C} 281-\mathrm{C} 286$ & $-172.30(15)$ \\
\hline $231-\mathrm{P} 2-\mathrm{C} 271-\mathrm{C} 272$ & $89(6)$ & $\mathrm{C} 286-\mathrm{C} 281-\mathrm{C} 282-\mathrm{C} 283$ & $-41(3)$ \\
\hline $\mathrm{C} 241-\mathrm{P} 2-\mathrm{C} 271-\mathrm{C} 272$ & $-52.60(13)$ & $\mathrm{P} 2-\mathrm{C} 281-\mathrm{C} 282-\mathrm{C} 283$ & $-179(2)$ \\
\hline $\mathrm{C} 281-\mathrm{P} 2-\mathrm{C} 271-\mathrm{C} 272$ & $-107(4)$ & $\mathrm{C} 281-\mathrm{C} 282-\mathrm{C} 283-\mathrm{C} 284$ & $2(4)$ \\
\hline $251-\mathrm{P} 2-\mathrm{C} 271-\mathrm{C} 272$ & $-60.70(15)$ & $\mathrm{C} 282-\mathrm{C} 283-\mathrm{C} 284-\mathrm{C} 285$ & $23(3)$ \\
\hline $61-\mathrm{P} 2-\mathrm{C} 271-\mathrm{C} 272$ & $-150(7)$ & $\mathrm{C} 283-\mathrm{C} 284-\mathrm{C} 285-\mathrm{C} 286$ & $-6(3)$ \\
\hline $221-\mathrm{P} 2-\mathrm{C} 271-\mathrm{C} 272$ & $50.90(14)$ & $\mathrm{C} 282-\mathrm{C} 281-\mathrm{C} 286-\mathrm{C} 285$ & $55(3)$ \\
\hline $\mathrm{NI} 2-\mathrm{P} 2-\mathrm{C} 271-\mathrm{C} 272$ & $176.90(11)$ & $\mathrm{P} 2-\mathrm{C} 281-\mathrm{C} 286-\mathrm{C} 285$ & $-157.20(19)$ \\
\hline $291-\mathrm{P} 2-\mathrm{C} 271-\mathrm{C} 276$ & $-81.00(11)$ & $\mathrm{C} 284-\mathrm{C} 285-\mathrm{C} 286-\mathrm{C} 281$ & $-36(3)$ \\
\hline $31-\mathrm{P} 2-\mathrm{C} 271-\mathrm{C} 276$ & $-43(6)$ & $\mathrm{C} 291-\mathrm{P} 2-\mathrm{C} 241-\mathrm{C} 246$ & $-13.4(8)$ \\
\hline $41-\mathrm{P} 2-\mathrm{C} 271-\mathrm{C} 276$ & $175.4(9)$ & $\mathrm{C} 271-\mathrm{P} 2-\mathrm{C} 241-\mathrm{C} 246$ & $82.3(8)$ \\
\hline 31-P2-C271-C276 & $121(4)$ & $\mathrm{C} 231-\mathrm{P} 2-\mathrm{C} 241-\mathrm{C} 246$ & $76.1(9)$ \\
\hline
\end{tabular}




\begin{tabular}{|c|c|c|c|}
\hline $\mathrm{C} 281-\mathrm{P} 2-\mathrm{C} 241-\mathrm{C} 246$ & $93.0(9)$ & $\mathrm{C} 251-\mathrm{C} 252-\mathrm{C} 253-\mathrm{C} 254$ & $0(2)$ \\
\hline $\mathrm{C} 251-\mathrm{P} 2-\mathrm{C} 241-\mathrm{C} 246$ & $165(4)$ & $\mathrm{C} 252-\mathrm{C} 253-\mathrm{C} 254-\mathrm{C} 255$ & $0(2)$ \\
\hline $\mathrm{C} 261-\mathrm{P} 2-\mathrm{C} 241-\mathrm{C} 246$ & $94.8(8)$ & $\mathrm{C} 253-\mathrm{C} 254-\mathrm{C} 255-\mathrm{C} 256$ & $0(2)$ \\
\hline $\mathrm{C} 221-\mathrm{P} 2-\mathrm{C} 241-\mathrm{C} 246$ & $-25.4(7)$ & $\mathrm{C} 252-\mathrm{C} 251-\mathrm{C} 256-\mathrm{C} 255$ & $0(2)$ \\
\hline $\mathrm{NI} 2-\mathrm{P} 2-\mathrm{C} 241-\mathrm{C} 246$ & $-145.5(5)$ & $\mathrm{P} 2-\mathrm{C} 251-\mathrm{C} 256-\mathrm{C} 255$ & $-169.3(9)$ \\
\hline $\mathrm{C} 291-\mathrm{P} 2-\mathrm{C} 241-\mathrm{C} 242$ & $169.1(7)$ & $\mathrm{C} 254-\mathrm{C} 255-\mathrm{C} 256-\mathrm{C} 251$ & $0.20(19)$ \\
\hline $\mathrm{C} 271-\mathrm{P} 2-\mathrm{C} 241-\mathrm{C} 242$ & $-95.2(7)$ & $\mathrm{C} 291-\mathrm{P} 2-\mathrm{C} 221-\mathrm{C} 226$ & $-162(4)$ \\
\hline $\mathrm{C} 231-\mathrm{P} 2-\mathrm{C} 241-\mathrm{C} 242$ & $-101.4(8)$ & $\mathrm{C} 271-\mathrm{P} 2-\mathrm{C} 221-\mathrm{C} 226$ & $-161.8(9)$ \\
\hline $\mathrm{C} 281-\mathrm{P} 2-\mathrm{C} 241-\mathrm{C} 242$ & $-84.5(9)$ & $\mathrm{C} 231-\mathrm{P} 2-\mathrm{C} 221-\mathrm{C} 226$ & $-167.5(9)$ \\
\hline $\mathrm{C} 251-\mathrm{P} 2-\mathrm{C} 241-\mathrm{C} 242$ & $-12(3)$ & $\mathrm{C} 241-\mathrm{P} 2-\mathrm{C} 221-\mathrm{C} 226$ & $-53.8(8)$ \\
\hline $\mathrm{C} 261-\mathrm{P} 2-\mathrm{C} 241-\mathrm{C} 242$ & $-82.7(8)$ & $\mathrm{C} 281-\mathrm{P} 2-\mathrm{C} 221-\mathrm{C} 226$ & $-156.30(11)$ \\
\hline $\mathrm{C} 221-\mathrm{P} 2-\mathrm{C} 241-\mathrm{C} 242$ & $157.1(5)$ & $\mathrm{C} 251-\mathrm{P} 2-\mathrm{C} 221-\mathrm{C} 226$ & $-52.2(8)$ \\
\hline $\mathrm{NI} 2-\mathrm{P} 2-\mathrm{C} 241-\mathrm{C} 242$ & $37.0(6)$ & $\mathrm{C} 261-\mathrm{P} 2-\mathrm{C} 221-\mathrm{C} 226$ & $-166.50(12)$ \\
\hline $\mathrm{C} 246-\mathrm{C} 241-\mathrm{C} 242-\mathrm{C} 243$ & $-1.6(1)$ & $\mathrm{NI} 2-\mathrm{P} 2-\mathrm{C} 221-\mathrm{C} 226$ & $68.6(8)$ \\
\hline $\mathrm{P} 2-\mathrm{C} 241-\mathrm{C} 242-\mathrm{C} 243$ & $176.1(5)$ & $\mathrm{C} 291-\mathrm{P} 2-\mathrm{C} 221-\mathrm{C} 222$ & $14(3)$ \\
\hline $\mathrm{C} 241-\mathrm{C} 242-\mathrm{C} 243-\mathrm{C} 244$ & $0.00(12)$ & $\mathrm{C} 271-\mathrm{P} 2-\mathrm{C} 221-\mathrm{C} 222$ & $14.5(1)$ \\
\hline $\mathrm{C} 242-\mathrm{C} 243-\mathrm{C} 244-\mathrm{C} 245$ & $2.90(17)$ & $\mathrm{C} 231-\mathrm{P} 2-\mathrm{C} 221-\mathrm{C} 222$ & $8.8(1)$ \\
\hline $\mathrm{C} 243-\mathrm{C} 244-\mathrm{C} 245-\mathrm{C} 246$ & $-4.10(17)$ & $\mathrm{C} 241-\mathrm{P} 2-\mathrm{C} 221-\mathrm{C} 222$ & $122.5(8)$ \\
\hline $\mathrm{C} 242-\mathrm{C} 241-\mathrm{C} 246-\mathrm{C} 245$ & $0.4(1)$ & $\mathrm{C} 281-\mathrm{P} 2-\mathrm{C} 221-\mathrm{C} 222$ & $19.90(12)$ \\
\hline $\mathrm{P} 2-\mathrm{C} 241-\mathrm{C} 246-\mathrm{C} 245$ & $-177.0(6)$ & $\mathrm{C} 251-\mathrm{P} 2-\mathrm{C} 221-\mathrm{C} 222$ & $124.0(8)$ \\
\hline $\mathrm{C} 244-\mathrm{C} 245-\mathrm{C} 246-\mathrm{C} 241$ & $2.50(13)$ & $\mathrm{C} 261-\mathrm{P} 2-\mathrm{C} 221-\mathrm{C} 222$ & $9.80(13)$ \\
\hline $\mathrm{C} 291-\mathrm{P} 2-\mathrm{C} 251-\mathrm{C} 256$ & $92.30(15)$ & $\mathrm{NI} 2-\mathrm{P} 2-\mathrm{C} 221-\mathrm{C} 222$ & $-115.1(7)$ \\
\hline $\mathrm{C} 271-\mathrm{P} 2-\mathrm{C} 251-\mathrm{C} 256$ & $-170.00(13)$ & $\mathrm{C} 226-\mathrm{C} 221-\mathrm{C} 222-\mathrm{C} 223$ & $-5.40(14)$ \\
\hline $\mathrm{C} 231-\mathrm{P} 2-\mathrm{C} 251-\mathrm{C} 256$ & $-175.00(13)$ & $\mathrm{P} 2-\mathrm{C} 221-C 222-\mathrm{C} 223$ & $177.9(9)$ \\
\hline $\mathrm{C} 241-\mathrm{P} 2-\mathrm{C} 251-\mathrm{C} 256$ & $91(4)$ & $\mathrm{C} 221-\mathrm{C} 222-\mathrm{C} 223-\mathrm{C} 224$ & $7(2)$ \\
\hline $\mathrm{C} 281-\mathrm{P} 2-\mathrm{C} 251-\mathrm{C} 256$ & $-160.60(15)$ & $\mathrm{C} 222-\mathrm{C} 223-\mathrm{C} 224-\mathrm{C} 225$ & $-4(2)$ \\
\hline $\mathrm{C} 261-\mathrm{P} 2-\mathrm{C} 251-\mathrm{C} 256$ & $-157.60(14)$ & $\mathrm{C} 223-\mathrm{C} 224-\mathrm{C} 225-\mathrm{C} 226$ & $1.10(16)$ \\
\hline $\mathrm{C} 221-\mathrm{P} 2-\mathrm{C} 251-\mathrm{C} 256$ & $79.90(14)$ & $\mathrm{C} 222-\mathrm{C} 221-\mathrm{C} 226-\mathrm{C} 225$ & $2.30(12)$ \\
\hline $\mathrm{NI} 2-\mathrm{P} 2-\mathrm{C} 251-\mathrm{C} 256$ & $-42.40(16)$ & $\mathrm{P} 2-\mathrm{C} 221-\mathrm{C} 226-\mathrm{C} 225$ & $178.9(7)$ \\
\hline $\mathrm{C} 291-\mathrm{P} 2-\mathrm{C} 251-\mathrm{C} 252$ & $-76.40(18)$ & $\mathrm{C} 224-\mathrm{C} 225-\mathrm{C} 226-\mathrm{C} 221$ & $-0.30(13)$ \\
\hline $\mathrm{C} 271-\mathrm{P} 2-\mathrm{C} 251-\mathrm{C} 252$ & $21.20(18)$ & $\mathrm{C} 271-\mathrm{P} 2-\mathrm{C} 291-\mathrm{C} 296$ & $-143.00(14)$ \\
\hline $\mathrm{C} 231-\mathrm{P} 2-\mathrm{C} 251-\mathrm{C} 252$ & $16(2)$ & $\mathrm{C} 231-\mathrm{P} 2-\mathrm{C} 291-\mathrm{C} 296$ & $-148.70(14)$ \\
\hline $\mathrm{C} 241-\mathrm{P} 2-\mathrm{C} 251-\mathrm{C} 252$ & $-78(3)$ & $\mathrm{C} 241-\mathrm{P} 2-\mathrm{C} 291-\mathrm{C} 296$ & $-37.20(14)$ \\
\hline $\mathrm{C} 281-\mathrm{P} 2-\mathrm{C} 251-\mathrm{C} 252$ & $30.70(18)$ & $\mathrm{C} 281-\mathrm{P} 2-\mathrm{C} 291-\mathrm{C} 296$ & $-138.00(14)$ \\
\hline $\mathrm{C} 261-\mathrm{P} 2-\mathrm{C} 251-\mathrm{C} 252$ & $33.60(18)$ & $\mathrm{C} 251-\mathrm{P} 2-\mathrm{C} 291-\mathrm{C} 296$ & $-37.40(15)$ \\
\hline $\mathrm{C} 221-\mathrm{P} 2-\mathrm{C} 251-\mathrm{C} 252$ & $-88.90(16)$ & $\mathrm{C} 261-\mathrm{P} 2-\mathrm{C} 291-\mathrm{C} 296$ & $-147.40(15)$ \\
\hline $\mathrm{NI} 2-\mathrm{P} 2-\mathrm{C} 251-\mathrm{C} 252$ & $148.80(14)$ & $\mathrm{C} 221-\mathrm{P} 2-\mathrm{C} 291-\mathrm{C} 296$ & $36(3)$ \\
\hline $\mathrm{C} 256-\mathrm{C} 251-\mathrm{C} 252-\mathrm{C} 253$ & $0(2)$ & $\mathrm{NI} 2-\mathrm{P} 2-\mathrm{C} 291-\mathrm{C} 296$ & $92.30(13)$ \\
\hline $\mathrm{P} 2-\mathrm{C} 251-\mathrm{C} 252-\mathrm{C} 253$ & $168.50(12)$ & $\mathrm{C} 271-\mathrm{P} 2-\mathrm{C} 291-\mathrm{C} 292$ & $57.10(14)$ \\
\hline
\end{tabular}




\begin{tabular}{|c|c|}
\hline $\mathrm{C} 231-\mathrm{P} 2-\mathrm{C} 291-\mathrm{C} 292$ & $51.50(13)$ \\
\hline $\mathrm{C} 241-\mathrm{P} 2-\mathrm{C} 291-\mathrm{C} 292$ & $162.90(12)$ \\
\hline $\mathrm{C} 281-\mathrm{P} 2-\mathrm{C} 291-\mathrm{C} 292$ & $62.10(15)$ \\
\hline $\mathrm{C} 251-\mathrm{P} 2-\mathrm{C} 291-\mathrm{C} 292$ & $162.70(11)$ \\
\hline $\mathrm{C} 261-\mathrm{P} 2-\mathrm{C} 291-\mathrm{C} 292$ & $52.80(16)$ \\
\hline $\mathrm{C} 221-\mathrm{P} 2-\mathrm{C} 291-\mathrm{C} 292$ & $-123(5)$ \\
\hline $\mathrm{NI} 2-\mathrm{P} 2-\mathrm{C} 291-\mathrm{C} 292$ & $-67.50(13)$ \\
\hline $\mathrm{C} 296-\mathrm{C} 291-\mathrm{C} 292-\mathrm{C} 293$ & $16(3)$ \\
\hline $\mathrm{P} 2-\mathrm{C} 291-\mathrm{C} 292-\mathrm{C} 293$ & $171.60(11)$ \\
\hline $\mathrm{C} 291-\mathrm{C} 292-\mathrm{C} 293-\mathrm{C} 294$ & $-6(2)$ \\
\hline $\mathrm{C} 292-\mathrm{C} 293-\mathrm{C} 294-\mathrm{C} 295$ & $-1(2)$ \\
\hline $\mathrm{C} 293-\mathrm{C} 294-\mathrm{C} 295-\mathrm{C} 296$ & $2(2)$ \\
\hline $\mathrm{C} 292-\mathrm{C} 291-\mathrm{C} 296-\mathrm{C} 295$ & $-15(3)$ \\
\hline $\mathrm{P} 2-\mathrm{C} 291-\mathrm{C} 296-\mathrm{C} 295$ & $-170.50(11)$ \\
\hline $\mathrm{C} 294-\mathrm{C} 295-\mathrm{C} 296-\mathrm{C} 291$ & $5(2)$ \\
\hline $\mathrm{C} 15-\mathrm{C} 14-\mathrm{C} 13 \mathrm{~A}-\mathrm{C} 17 \mathrm{~A}$ & $-0.9(4)$ \\
\hline $\mathrm{C} 15-\mathrm{C} 14-\mathrm{C} 13 \mathrm{~A}-\mathrm{C} 13$ & $-178.5(3)$ \\
\hline $\mathrm{C} 15-\mathrm{C} 14-\mathrm{C} 13 \mathrm{~A}-\mathrm{NI} 1$ & $-93.9(4)$ \\
\hline $\mathrm{C} 12-\mathrm{C} 13-\mathrm{C} 13 \mathrm{~A}-\mathrm{C} 14$ & $-174.1(3)$ \\
\hline $\mathrm{NI1}-\mathrm{C} 13-\mathrm{C} 13 \mathrm{~A}-\mathrm{C} 14$ & $119.6(3)$ \\
\hline $\mathrm{C} 12-\mathrm{C} 13-\mathrm{C} 13 \mathrm{~A}-\mathrm{C} 17 \mathrm{~A}$ & $8.0(3)$ \\
\hline $\mathrm{NI} 1-\mathrm{C} 13-\mathrm{C} 13 \mathrm{~A}-\mathrm{C} 17 \mathrm{~A}$ & $-58.27(18)$ \\
\hline $\mathrm{C} 12-\mathrm{C} 13-\mathrm{C} 13 \mathrm{~A}-\mathrm{NI} 1$ & $66.31(17)$ \\
\hline $\mathrm{C} 13-\mathrm{NI} 1-\mathrm{C} 13 \mathrm{~A}-\mathrm{C} 14$ & $-122.4(4)$ \\
\hline $\mathrm{C} 12-\mathrm{N} 11-\mathrm{C} 13 \mathrm{~A}-\mathrm{C} 14$ & $-163.8(4)$ \\
\hline $\mathrm{C} 11-\mathrm{NI} 1-\mathrm{C} 13 \mathrm{~A}-\mathrm{C} 14$ & $151.6(4)$ \\
\hline $\mathrm{CL} 1-\mathrm{NI} 1-\mathrm{C} 13 \mathrm{~A}-\mathrm{C} 14$ & $83.0(4)$ \\
\hline $\mathrm{P} 1-\mathrm{N} 11-\mathrm{C} 13 \mathrm{~A}-\mathrm{C} 14$ & $-33.8(4)$ \\
\hline $\mathrm{C} 17 \mathrm{~A}-\mathrm{NI} 1-\mathrm{C} 13 \mathrm{~A}-\mathrm{C} 14$ & $116.0(4)$ \\
\hline $\mathrm{C} 13-\mathrm{NI} 1-\mathrm{C} 13 \mathrm{~A}-\mathrm{C} 17 \mathrm{~A}$ & $121.6(2)$ \\
\hline $\mathrm{C} 12-\mathrm{NI} 1-\mathrm{C} 13 \mathrm{~A}-\mathrm{C} 17 \mathrm{~A}$ & $80.23(17)$ \\
\hline $\mathrm{C} 11-\mathrm{NI} 1-\mathrm{C} 13 \mathrm{~A}-\mathrm{C} 17 \mathrm{~A}$ & $35.64(15)$ \\
\hline $\mathrm{CL} 1-\mathrm{NI} 1-\mathrm{C} 13 \mathrm{~A}-\mathrm{C} 17 \mathrm{~A}$ & $-33.0(2)$ \\
\hline $\mathrm{P} 1-\mathrm{NI} 1-\mathrm{C} 13 \mathrm{~A}-\mathrm{C} 17 \mathrm{~A}$ & $-149.81(14)$ \\
\hline $\mathrm{C} 12-\mathrm{NI} 1-\mathrm{C} 13 \mathrm{~A}-\mathrm{C} 13$ & $-41.36(16)$ \\
\hline $\mathrm{C} 11-\mathrm{NI} 1-\mathrm{C} 13 \mathrm{~A}-\mathrm{C} 13$ & $-85.95(17)$ \\
\hline $\mathrm{CL} 1-\mathrm{NI} 1-\mathrm{C} 13 \mathrm{~A}-\mathrm{C} 13$ & $-154.56(14)$ \\
\hline $\mathrm{P} 1-\mathrm{NI} 1-\mathrm{C} 13 \mathrm{~A}-\mathrm{C} 13$ & $88.60(15)$ \\
\hline $\mathrm{C} 17 \mathrm{~A}-\mathrm{NI} 1-\mathrm{C} 13 \mathrm{~A}-\mathrm{C} 13$ & $-121.6(2)$ \\
\hline
\end{tabular}

\begin{tabular}{|c|c|}
\hline $\mathrm{C} 16-\mathrm{C} 17-\mathrm{C} 17 \mathrm{~A}-\mathrm{C} 13 \mathrm{~A}$ & $2.8(4)$ \\
\hline $\mathrm{C} 16-\mathrm{C} 17-\mathrm{C} 17 \mathrm{~A}-\mathrm{C} 11$ & $-179.9(3)$ \\
\hline $\mathrm{C} 16-\mathrm{C} 17-\mathrm{C} 17 \mathrm{~A}-\mathrm{NI} 1$ & $93.0(3)$ \\
\hline $\mathrm{C} 14-\mathrm{C} 13 \mathrm{~A}-\mathrm{C} 17 \mathrm{~A}-\mathrm{C} 17$ & $-1.8(4)$ \\
\hline $\mathrm{C} 13-\mathrm{C} 13 \mathrm{~A}-\mathrm{C} 17 \mathrm{~A}-\mathrm{C} 17$ & $176.3(2)$ \\
\hline $\mathrm{NI} 1-\mathrm{C} 13 \mathrm{~A}-\mathrm{C} 17 \mathrm{~A}-\mathrm{C} 17$ & $125.3(2)$ \\
\hline $\mathrm{C} 14-\mathrm{C} 13 \mathrm{~A}-\mathrm{C} 17 \mathrm{~A}-\mathrm{C} 11$ & $-179.7(2)$ \\
\hline $\mathrm{C} 13-\mathrm{C} 13 \mathrm{~A}-\mathrm{C} 17 \mathrm{~A}-\mathrm{C} 11$ & $-1.5(3)$ \\
\hline $\mathrm{NI} 1-\mathrm{C} 13 \mathrm{~A}-\mathrm{C} 17 \mathrm{~A}-\mathrm{C} 11$ & $-52.56(15)$ \\
\hline $\mathrm{C} 14-\mathrm{C} 13 \mathrm{~A}-\mathrm{C} 17 \mathrm{~A}-\mathrm{NI} 1$ & $-127.2(3)$ \\
\hline $\mathrm{C} 13-\mathrm{C} 13 \mathrm{~A}-\mathrm{C} 17 \mathrm{~A}-\mathrm{NI} 1$ & $51.02(16)$ \\
\hline $\mathrm{C} 12-\mathrm{C} 11-\mathrm{C} 17 \mathrm{~A}-\mathrm{C} 17$ & $177.1(3)$ \\
\hline $\mathrm{SI} 1-\mathrm{C} 11-\mathrm{C} 17 \mathrm{~A}-\mathrm{C} 17$ & $8.8(4)$ \\
\hline $\mathrm{NI} 1-\mathrm{C} 11-\mathrm{C} 17 \mathrm{~A}-\mathrm{C} 17$ & $-119.9(3)$ \\
\hline $\mathrm{C} 12-\mathrm{C} 11-\mathrm{C} 17 \mathrm{~A}-\mathrm{C} 13 \mathrm{~A}$ & $-5.3(3)$ \\
\hline $\mathrm{SI} 1-\mathrm{C} 11-\mathrm{C} 17 \mathrm{~A}-\mathrm{C} 13 \mathrm{~A}$ & $-173.63(17)$ \\
\hline $\mathrm{NI1}-\mathrm{C} 11-\mathrm{C} 17 \mathrm{~A}-\mathrm{C} 13 \mathrm{~A}$ & $57.63(17)$ \\
\hline $\mathrm{C} 12-\mathrm{C} 11-\mathrm{C} 17 \mathrm{~A}-\mathrm{NI} 1$ & $-62.97(15)$ \\
\hline $\mathrm{SI} 1-\mathrm{C} 11-\mathrm{C} 17 \mathrm{~A}-\mathrm{NI} 1$ & $128.7(2)$ \\
\hline $\mathrm{C} 13-\mathrm{NI} 1-\mathrm{C} 17 \mathrm{~A}-\mathrm{C} 17$ & $-150.1(3)$ \\
\hline $\mathrm{C} 12-\mathrm{NI} 1-\mathrm{C} 17 \mathrm{~A}-\mathrm{C} 17$ & $165.0(3)$ \\
\hline $\mathrm{C} 11-\mathrm{NI} 1-\mathrm{C} 17 \mathrm{~A}-\mathrm{C} 17$ & $123.6(3)$ \\
\hline $\mathrm{CL} 1-\mathrm{NI} 1-\mathrm{C} 17 \mathrm{~A}-\mathrm{C} 17$ & $43.5(3)$ \\
\hline $\mathrm{P} 1-\mathrm{NI} 1-\mathrm{C} 17 \mathrm{~A}-\mathrm{C} 17$ & $-71.8(3)$ \\
\hline $\mathrm{C} 13 \mathrm{~A}-\mathrm{NI} 1-\mathrm{C} 17 \mathrm{~A}-\mathrm{C} 17$ & $-113.9(3)$ \\
\hline $\mathrm{C} 13-\mathrm{NI} 1-\mathrm{C} 17 \mathrm{~A}-\mathrm{C} 13 \mathrm{~A}$ & $-36.15(18)$ \\
\hline $\mathrm{C} 12-\mathrm{NI} 1-\mathrm{C} 17 \mathrm{~A}-\mathrm{C} 13 \mathrm{~A}$ & $-81.07(19)$ \\
\hline $\mathrm{C} 11-\mathrm{NI} 1-\mathrm{C} 17 \mathrm{~A}-\mathrm{C} 13 \mathrm{~A}$ & $-122.5(2)$ \\
\hline $\mathrm{CL} 1-\mathrm{NI} 1-\mathrm{C} 17 \mathrm{~A}-\mathrm{C} 13 \mathrm{~A}$ & $157.45(16)$ \\
\hline $\mathrm{P} 1-\mathrm{NI} 1-\mathrm{C} 17 \mathrm{~A}-\mathrm{C} 13 \mathrm{~A}$ & $42.1(2)$ \\
\hline $\mathrm{C} 13-\mathrm{N} 11-\mathrm{C} 17 \mathrm{~A}-\mathrm{C} 11$ & $86.34(17)$ \\
\hline $\mathrm{C} 12-\mathrm{NI} 1-\mathrm{C} 17 \mathrm{~A}-\mathrm{C} 11$ & $41.43(15)$ \\
\hline $\mathrm{CL} 1-\mathrm{NI} 1-\mathrm{C} 17 \mathrm{~A}-\mathrm{C} 11$ & $-80.05(14)$ \\
\hline $\mathrm{P} 1-\mathrm{NI} 1-\mathrm{C} 17 \mathrm{~A}-\mathrm{C} 11$ & $164.60(11)$ \\
\hline $\mathrm{C} 13 \mathrm{~A}-\mathrm{NI} 1-\mathrm{C} 17 \mathrm{~A}-\mathrm{C} 11$ & $122.5(2)$ \\
\hline $\mathrm{C} 25-\mathrm{C} 24-\mathrm{C} 23 \mathrm{~A}-\mathrm{C} 27 \mathrm{~A}$ & $1.0(5)$ \\
\hline $\mathrm{C} 25-\mathrm{C} 24-\mathrm{C} 23 \mathrm{~A}-\mathrm{C} 23$ & $178.8(4)$ \\
\hline $\mathrm{C} 25-\mathrm{C} 24-\mathrm{C} 23 \mathrm{~A}-\mathrm{NI} 2$ & $94.2(5)$ \\
\hline $\mathrm{C} 22-\mathrm{C} 23-\mathrm{C} 23 \mathrm{~A}-\mathrm{C} 27 \mathrm{~A}$ & $-7.8(3)$ \\
\hline
\end{tabular}




\begin{tabular}{|c|c|c|c|}
\hline $\mathrm{NI} 2-\mathrm{C} 23-\mathrm{C} 23 \mathrm{~A}-\mathrm{C} 27 \mathrm{~A}$ & $58.9(2)$ & $\mathrm{NI} 2-\mathrm{C} 23 \mathrm{~A}-\mathrm{C} 27 \mathrm{~A}-\mathrm{C} 21$ & $51.41(17)$ \\
\hline $\mathrm{C} 22-\mathrm{C} 23-\mathrm{C} 23 \mathrm{~A}-\mathrm{C} 24$ & $174.3(3)$ & $\mathrm{C} 24-\mathrm{C} 23 \mathrm{~A}-\mathrm{C} 27 \mathrm{~A}-\mathrm{NI} 2$ & $128.0(3)$ \\
\hline $\mathrm{NI} 2-\mathrm{C} 23-\mathrm{C} 23 \mathrm{~A}-\mathrm{C} 24$ & $-119.0(4)$ & $\mathrm{C} 23-\mathrm{C} 23 \mathrm{~A}-\mathrm{C} 27 \mathrm{~A}-\mathrm{NI} 2$ & $-50.33(18)$ \\
\hline $\mathrm{C} 22-\mathrm{C} 23-\mathrm{C} 23 \mathrm{~A}-\mathrm{NI} 2$ & $-66.7(2)$ & $\mathrm{C} 22-\mathrm{C} 21-\mathrm{C} 27 \mathrm{~A}-\mathrm{C} 27$ & $-176.4(3)$ \\
\hline $\mathrm{C} 23-\mathrm{NI} 2-\mathrm{C} 23 \mathrm{~A}-\mathrm{C} 27 \mathrm{~A}$ & $-121.6(3)$ & $\mathrm{SI} 2-\mathrm{C} 21-\mathrm{C} 27 \mathrm{~A}-\mathrm{C} 27$ & $-8.7(4)$ \\
\hline $\mathrm{C} 22-\mathrm{NI} 2-\mathrm{C} 23 \mathrm{~A}-\mathrm{C} 27 \mathrm{~A}$ & $-79.7(2)$ & $\mathrm{NI} 2-\mathrm{C} 21-\mathrm{C} 27 \mathrm{~A}-\mathrm{C} 27$ & $121.0(3)$ \\
\hline $\mathrm{C} 21-\mathrm{NI} 2-\mathrm{C} 23 \mathrm{~A}-\mathrm{C} 27 \mathrm{~A}$ & $-35.17(19)$ & $\mathrm{C} 22-\mathrm{C} 21-\mathrm{C} 27 \mathrm{~A}-\mathrm{C} 23 \mathrm{~A}$ & $5.9(3)$ \\
\hline $\mathrm{CL} 2 \mathrm{~A}-\mathrm{NI} 2-\mathrm{C} 23 \mathrm{~A}-\mathrm{C} 27 \mathrm{~A}$ & $43.6(2)$ & $\mathrm{SI} 2-\mathrm{C} 21-\mathrm{C} 27 \mathrm{~A}-\mathrm{C} 23 \mathrm{~A}$ & $173.66(19)$ \\
\hline $\mathrm{P} 2-\mathrm{NI} 2-\mathrm{C} 23 \mathrm{~A}-\mathrm{C} 27 \mathrm{~A}$ & $157.01(18)$ & $\mathrm{NI} 2-\mathrm{C} 21-\mathrm{C} 27 \mathrm{~A}-\mathrm{C} 23 \mathrm{~A}$ & $-56.65(19)$ \\
\hline $\mathrm{CL} 2 \mathrm{~B}-\mathrm{NI} 2-\mathrm{C} 23 \mathrm{~A}-\mathrm{C} 27 \mathrm{~A}$ & $27.3(3)$ & $\mathrm{C} 22-\mathrm{C} 21-\mathrm{C} 27 \mathrm{~A}-\mathrm{NI} 2$ & $62.58(17)$ \\
\hline $\mathrm{C} 23-\mathrm{NI} 2-\mathrm{C} 23 \mathrm{~A}-\mathrm{C} 24$ & $124.0(5)$ & $\mathrm{SI} 2-\mathrm{C} 21-\mathrm{C} 27 \mathrm{~A}-\mathrm{NI} 2$ & $-129.7(2)$ \\
\hline $\mathrm{C} 22-\mathrm{NI} 2-\mathrm{C} 23 \mathrm{~A}-\mathrm{C} 24$ & $165.9(4)$ & $\mathrm{C} 23-\mathrm{NI} 2-\mathrm{C} 27 \mathrm{~A}-\mathrm{C} 27$ & $149.9(4)$ \\
\hline $\mathrm{C} 21-\mathrm{NI} 2-\mathrm{C} 23 \mathrm{~A}-\mathrm{C} 24$ & $-149.6(4)$ & $\mathrm{C} 22-\mathrm{NI} 2-\mathrm{C} 27 \mathrm{~A}-\mathrm{C} 27$ & $-164.4(4)$ \\
\hline $\mathrm{CL} 2 \mathrm{~A}-\mathrm{NI} 2-\mathrm{C} 23 \mathrm{~A}-\mathrm{C} 24$ & $-70.8(4)$ & $\mathrm{C} 21-\mathrm{NI} 2-\mathrm{C} 27 \mathrm{~A}-\mathrm{C} 27$ & $-123.3(4)$ \\
\hline $\mathrm{P} 2-\mathrm{NI} 2-\mathrm{C} 23 \mathrm{~A}-\mathrm{C} 24$ & $42.6(4)$ & $\mathrm{CL} 2 \mathrm{~A}-\mathrm{NI} 2-\mathrm{C} 27 \mathrm{~A}-\mathrm{C} 27$ & $-33.4(4)$ \\
\hline $\mathrm{CL} 2 \mathrm{~B}-\mathrm{NI} 2-\mathrm{C} 23 \mathrm{~A}-\mathrm{C} 24$ & $-87.1(5)$ & $\mathrm{P} 2-\mathrm{NI} 2-\mathrm{C} 27 \mathrm{~A}-\mathrm{C} 27$ & $79.2(4)$ \\
\hline $\mathrm{C} 27 \mathrm{~A}-\mathrm{NI} 2-\mathrm{C} 23 \mathrm{~A}-\mathrm{C} 24$ & $-114.4(5)$ & $\mathrm{CL} 2 \mathrm{~B}-\mathrm{NI} 2-\mathrm{C} 27 \mathrm{~A}-\mathrm{C} 27$ & $-48.5(4)$ \\
\hline $\mathrm{C} 22-\mathrm{NI} 2-\mathrm{C} 23 \mathrm{~A}-\mathrm{C} 23$ & $41.93(19)$ & $\mathrm{C} 23 \mathrm{~A}-\mathrm{NI} 2-\mathrm{C} 27 \mathrm{~A}-\mathrm{C} 27$ & $113.8(4)$ \\
\hline $\mathrm{C} 21-\mathrm{NI} 2-\mathrm{C} 23 \mathrm{~A}-\mathrm{C} 23$ & $86.4(2)$ & $\mathrm{C} 23-\mathrm{NI} 2-\mathrm{C} 27 \mathrm{~A}-\mathrm{C} 23 \mathrm{~A}$ & $36.2(2)$ \\
\hline $\mathrm{CL} 2 \mathrm{~A}-\mathrm{NI} 2-\mathrm{C} 23 \mathrm{~A}-\mathrm{C} 23$ & $165.21(17)$ & $\mathrm{C} 22-\mathrm{NI} 2-\mathrm{C} 27 \mathrm{~A}-\mathrm{C} 23 \mathrm{~A}$ & $81.8(2)$ \\
\hline $\mathrm{P} 2-\mathrm{NI} 2-\mathrm{C} 23 \mathrm{~A}-\mathrm{C} 23$ & $-81.39(19)$ & $\mathrm{C} 21-\mathrm{NI} 2-\mathrm{C} 27 \mathrm{~A}-\mathrm{C} 23 \mathrm{~A}$ & $122.9(3)$ \\
\hline $\mathrm{CL} 2 \mathrm{~B}-\mathrm{NI} 2-\mathrm{C} 23 \mathrm{~A}-\mathrm{C} 23$ & $148.9(3)$ & $\mathrm{CL} 2 \mathrm{~A}-\mathrm{NI} 2-\mathrm{C} 27 \mathrm{~A}-\mathrm{C} 23 \mathrm{~A}$ & $-147.1(2)$ \\
\hline $\mathrm{C} 27 \mathrm{~A}-\mathrm{NI} 2-\mathrm{C} 23 \mathrm{~A}-\mathrm{C} 23$ & $121.6(3)$ & $\mathrm{P} 2-\mathrm{NI} 2-\mathrm{C} 27 \mathrm{~A}-\mathrm{C} 23 \mathrm{~A}$ & $-34.5(3)$ \\
\hline $\mathrm{C} 26-\mathrm{C} 27-\mathrm{C} 27 \mathrm{~A}-\mathrm{C} 23 \mathrm{~A}$ & $-2.6(5)$ & $\mathrm{CL} 2 \mathrm{~B}-\mathrm{NI} 2-\mathrm{C} 27 \mathrm{~A}-\mathrm{C} 23 \mathrm{~A}$ & $-162.2(2)$ \\
\hline $\mathrm{C} 26-\mathrm{C} 27-\mathrm{C} 27 \mathrm{~A}-\mathrm{C} 21$ & $180.0(4)$ & $\mathrm{C} 23-\mathrm{NI} 2-\mathrm{C} 27 \mathrm{~A}-\mathrm{C} 21$ & $-86.7(2)$ \\
\hline $\mathrm{C} 26-\mathrm{C} 27-\mathrm{C} 27 \mathrm{~A}-\mathrm{NI} 2$ & $-92.8(5)$ & $\mathrm{C} 22-\mathrm{NI} 2-\mathrm{C} 27 \mathrm{~A}-\mathrm{C} 21$ & $-41.06(18)$ \\
\hline $\mathrm{C} 24-\mathrm{C} 23 \mathrm{~A}-\mathrm{C} 27 \mathrm{~A}-\mathrm{C} 27$ & $1.4(4)$ & $\mathrm{CL} 2 \mathrm{~A}-\mathrm{NI} 2-\mathrm{C} 27 \mathrm{~A}-\mathrm{C} 21$ & $89.98(16)$ \\
\hline $\mathrm{C} 23-\mathrm{C} 23 \mathrm{~A}-\mathrm{C} 27 \mathrm{~A}-\mathrm{C} 27$ & $-176.9(3)$ & $\mathrm{P} 2-\mathrm{NI} 2-\mathrm{C} 27 \mathrm{~A}-\mathrm{C} 21$ & $-157.43(14)$ \\
\hline $\mathrm{NI} 2-\mathrm{C} 23 \mathrm{~A}-\mathrm{C} 27 \mathrm{~A}-\mathrm{C} 27$ & $-126.6(3)$ & $\mathrm{CL} 2 \mathrm{~B}-\mathrm{NI} 2-\mathrm{C} 27 \mathrm{~A}-\mathrm{C} 21$ & $74.9(2)$ \\
\hline $\mathrm{C} 24-\mathrm{C} 23 \mathrm{~A}-\mathrm{C} 27 \mathrm{~A}-\mathrm{C} 21$ & $179.4(3)$ & $\mathrm{C} 23 \mathrm{~A}-\mathrm{NI} 2-\mathrm{C} 27 \mathrm{~A}-\mathrm{C} 21$ & $-122.9(3)$ \\
\hline $\mathrm{C} 23-\mathrm{C} 23 \mathrm{~A}-\mathrm{C} 27 \mathrm{~A}-\mathrm{C} 21$ & $1.1(3)$ & & \\
\hline
\end{tabular}


The structure was solved by direct method with ShelxS-97. All non-H atoms were refined by full-matrix least-squares with anisotropic displacement parameters while $\mathrm{H}$ atoms were placed in idealized position.

Data collection: SAINT (Bruker, 1999). Cell refinement: SMART (Bruker, 1999). Data reduction: SAINT (Bruker, 1999). Program(s) used to solve structure: SHELXS97 (Sheldrick, 1997). Program(s) used to refine structure: SHELXL97 (Sheldrick, 1997). Molecular graphics: XP (Bruker, 1999). Software used to prepare material for publication: UdMX (local program).

We are grateful to the Natural Sciences and Engineering Research Council of Canada and the Ministère de l'Education du Québec for financial support. 
Supplementary data for this paper are available from the IUCr electronic archives (Reference: PREVIEW). Services for accessing these data are described at the back of the journal.

\section{References}

Allen, F. H. \& Kennard, O. (1993). Chem. Des. Autom. News., 8, 31-37.

Altomare, A., Burla, M. C., Camalli, M., Cascarano, G. L., Giacovazzo, C. Guagliardi, A., Moliterni, A. G. G., Polidori, G. \& Spagna, R. (1999). J. Appl. Cryst. 32, 115-119.

Beurskens, P. T., Admiraal, G., Beurskens, G., Bosman, W. P., García-Granda, S., Gould, R. O., Smits, J. M. M. \& Smykalla, C. (1999). The DIRDIF99 program system, Technical Report of the Crystallography Laboratory, University of Nijmegen, The Netherlands.

Boer, J. L. de \& Duisenberg, A. J. M. (1984). Acta Cryst. A40, C-410.

Boeyens, J. C. A. (1978). J. Cryst. Mol. Struct. 8, 317-320.

Cremer, D. \& Pople, J. A. (1975). J. Am. Chem. Soc. 97, 1354-1358.

Enraf-Nonius (1989). CAD-4 Software. Version 5. Enraf-Nonius, Delft, The Netherlands.

Farrugia, L. J. (1997). ORTEP3 for Window, J. Appl. Cryst. 30, 565.

Farrugia, L. J. (1999). WinGX J. App. Cryst. 32, 837-838.

Flack, H. D. (1983). Acta Cryst. A39, 876-881.

Flack, H. D. \& Schwarzenbach, D. (1988). Acta Cryst. A44, 499-506.

LePage, Y. (1987). J. Appl. Cryst. 20, 264-269.

Mackay, A. L. (1984). Acta Cryst. A40, 165-166.

Meulenaer, J. de \& Tompa, H. (1965). Acta Cryst. 19, 1014-1018.

Nonius (1998). Collect Software, Nonius B. V., Delft, The Netherlands.

North, A. C. T., Phillips, D. C. \& Mathews, F. S. (1968). Acta Cryst. A24, 351-359.

Otwinowski, Z. \& Minor, W. (1997). Methods in Enzymology, Vol. 276, Macromolecular Crystallography, Part A, edited by C. W. Carter \& R. M. Sweet, pp. 307-326. London: Academic Press.

SAINT Release 6.06 (1999) Integration Software for Single Crystal Data. Bruker AXS Inc., Madison, WI 53719-1173.

Sheldrick, G. M. (1996). SADABS, Bruker Area Detector Absorption Corrections. Bruker AXS Inc., Madison, WI 53719-1173.

Sheldrick, G. M. (1986). SHELXS86. Program for Crystal Structure solution. University of Göttingen, Germany.

Sheldrick, G. M. (1997). SHELXS97. Program for Crystal Structure solution. University of Göttingen, Germany.

Sheldrick, G. M. (1997). SHELXL97. Program for crystal structure refinement. University of Göttingen, Germany.

SHELXTL (1997) Release 5.10; The Complete Software Package for Single Crystal Structure Determination. Bruker AXS Inc., Madison, WI 53719-1173.

SMART (1999) Release 5.059; Bruker Molecular Analysis Research Tool. Bruker AXS Inc., Madison, WI 53719-1173. 
Spek, A. L. (1987). Acta Cryst. C43, 1233-1235.

Spek, A. L. (1988). J. Appl. Cryst. 21, 578-579.

Spek, A. L. (1990). Acta Cryst. A46, C-34.

Spek, A. L. (1997). HELENA, Program for Datareduction, Utrecht University, The Netherlands.

Spek, A. L. (1994). Am. Crystallogr. Assoc.-Abstracts, 22, 66.

Spek, A. L. (2000). PLATON, Molecular Geometry Program, 2000 version. University of Utrecht, Utrecht, Holland.

Watkin, D. J., Prout, C. K., Carruthers, J. R., Betteridge, P. W. \& Cooper R. I. (2001) CRYSTALS Issue 11. Chemical Crystallography Laboratory, OXFORD, UK.

Watkin, D. J., Prout, C. K., Pearce, L. J. (1996). CAMERON, Chemical Crystallography Laboratory, University of Oxford, Oxford.

Watkin, D. J., Prout, C. K., Lilley, P. M.deQ. (1994), RC93, Chemical Crystallography Laboratory, University of Oxford, Oxford.

Wilson, A. J. C. (1992). Ed. International Tables for Crystallography, Volume C, Kluwer Academic Publishers, Dordrecht, The Netherlands.

XPREP (1997) Release 5.10; X-ray data Preparation and Reciprocal space Exploration Program. Bruker AXS Inc., Madison, WI 53719-1173.

Fig 1 ORTEP view of the title compound. Thermal ellipsoids are shown at $30 \%$ probability levels.

Table 1. Selected geometric parameters $\left(\AA{ }^{\circ}\right)$ for the title compound. 


\section{Supplementary data}

The tables of data shown below are not normally printed in Acta Cryst. Section $C$ but the data will be available electronically via the online contents pages at

http://journals.iucr.org/c/journalhomepage.html

Table S1. Fractional atomic coordinates and equivalent isotropic displacement parameters $\left(\AA^{2}\right)$

\begin{tabular}{|c|c|c|c|c|c|}
\hline & Occupancy & $x$ & $y$ & $z$ & $U_{\text {eq }}$ \\
\hline Ni1 & 1 & $0.38644(4)$ & $0.23631(3)$ & $0.07394(2)$ & $0.03729(12)$ \\
\hline Cl1 & 1 & $0.21305(6)$ & $0.29971(5)$ & 0.14271 & $0.04617(16)$ \\
\hline $\mathrm{P} 1$ & 1 & $0.48051(6)$ & 0.19757 (4) & $0.15989(3)$ & $0.03635(14)$ \\
\hline Si1 & 1 & $0.16930(7)$ & $0.29188(5)$ & $-0.05036(4)$ & $0.04025(16)$ \\
\hline C11 & 1 & $0.3400(2)$ & $0.26827(16)$ & $-0.02143(13)$ & $0.0366(5)$ \\
\hline $\mathrm{C} 12$ & 1 & $0.3889(3)$ & 0.17897 (19) & $-0.04616(14)$ & $0.0474(6)$ \\
\hline H12 & 1 & 0.3412 & 0.1243 & -0.0785 & 0.057 \\
\hline $\mathrm{C} 13$ & 1 & $0.5195(3)$ & $0.1853(2)$ & $-0.01481(17)$ & $0.0568(8)$ \\
\hline H13 & 1 & 0.5677 & 0.1362 & -0.0149 & 0.068 \\
\hline C14 & 1 & $0.6922(3)$ & $0.3242(4)$ & $0.0500(2)$ & $0.0777(12)$ \\
\hline H14 & 1 & 0.7655 & 0.2903 & 0.0525 & 0.093 \\
\hline C15 & 1 & $0.7049(4)$ & $0.4174(4)$ & $0.0778(2)$ & $0.0944(16)$ \\
\hline H15 & 1 & 0.7893 & 0.4475 & 0.0977 & 0.113 \\
\hline C16 & 1 & $0.5961(4)$ & $0.4691(3)$ & $0.0773(2)$ & $0.0775(11)$ \\
\hline H16 & 1 & 0.6076 & 0.5332 & 0.0980 & 0.093 \\
\hline $\mathrm{C} 17$ & 1 & $0.4712(3)$ & $0.4272(2)$ & $0.04674(16)$ & $0.0548(7)$ \\
\hline H17 & 1 & 0.3975 & 0.4623 & 0.0474 & 0.066 \\
\hline C111 & 1 & $0.1856(4)$ & $0.2898(2)$ & $-0.14949(17)$ & $0.0620(8)$ \\
\hline H11D & 1 & 0.0976 & 0.2966 & -0.1686 & 0.093 \\
\hline $\mathrm{H} 11 \mathrm{E}$ & 1 & 0.2206 & 0.2321 & -0.1844 & 0.093 \\
\hline $\mathrm{H} 11 \mathrm{~F}$ & 1 & 0.2471 & 0.3398 & -0.1468 & 0.093 \\
\hline $\mathrm{C} 112$ & 1 & $0.1094(3)$ & $0.4049(2)$ & $0.01586(19)$ & $0.0625(8)$ \\
\hline H11A & 1 & 0.0155 & 0.4089 & 0.0033 & 0.094 \\
\hline H11B & 1 & 0.1638 & 0.4530 & 0.0099 & 0.094 \\
\hline $\mathrm{H} 11 \mathrm{C}$ & 1 & 0.1174 & 0.4118 & 0.0691 & 0.094 \\
\hline C113 & 1 & $0.0476(3)$ & $0.1982(2)$ & $-0.0550(2)$ & $0.0632(8)$ \\
\hline $\mathrm{H} 11 \mathrm{G}$ & 1 & 0.0374 & 0.2003 & -0.0034 & 0.095 \\
\hline $\mathrm{H} 11 \mathrm{H}$ & 1 & 0.0813 & 0.1397 & -0.0891 & 0.095 \\
\hline H11I & 1 & -0.0393 & 0.2058 & -0.0748 & 0.095 \\
\hline $\mathrm{C} 121$ & 1 & $0.6496(2)$ & $0.15551(16)$ & $0.13682(14)$ & $0.0352(5)$ \\
\hline $\mathrm{C} 122$ & 1 & $0.7637(3)$ & $0.2111(2)$ & $0.1691(2)$ & $0.0593(8)$ \\
\hline H122 & 1 & 0.7550 & 0.2706 & 0.2077 & 0.071 \\
\hline $\mathrm{C} 123$ & 1 & $0.8911(3)$ & $0.1802(3)$ & $0.1453(3)$ & $0.0746(10)$ \\
\hline H123 & 1 & 0.9679 & 0.2192 & 0.1674 & 0.090 \\
\hline $\mathrm{C} 124$ & 1 & $0.9062(3)$ & $0.0939(3)$ & $0.0904(2)$ & $0.0657(9)$ \\
\hline H124 & 1 & 0.9931 & 0.0730 & 0.0757 & 0.079 \\
\hline $\mathrm{C} 125$ & 1 & $0.7947(3)$ & $0.0378(2)$ & $0.0565(2)$ & $0.0694(9)$ \\
\hline H125 & 1 & 0.8047 & -0.0214 & 0.0177 & 0.083 \\
\hline $\mathrm{C} 126$ & 1 & $0.6672(3)$ & $0.0683(2)$ & $0.0796(2)$ & $0.0563(7)$ \\
\hline H126 & 1 & 0.5909 & 0.0294 & 0.0561 & 0.068 \\
\hline C131 & 0.75 & $0.4993(12)$ & $0.2897(3)$ & $0.2568(3)$ & $0.0395(16)$ \\
\hline $\mathrm{C} 132$ & 0.75 & $0.4920(8)$ & $0.3782(3)$ & $0.2652(4)$ & $0.0520(16)$ \\
\hline H132 & 0.75 & 0.4668 & 0.3910 & 0.2230 & 0.062 \\
\hline $\mathrm{C} 133$ & 0.75 & $0.5227(8)$ & $0.4479(4)$ & $0.3372(4)$ & $0.070(2)$ \\
\hline H133 & 0.75 & 0.5231 & 0.5091 & 0.3435 & 0.084 \\
\hline $\mathrm{C} 134$ & 0.75 & $0.5528(13)$ & $0.4291(6)$ & $0.3994(6)$ & $0.070(3)$ \\
\hline H134 & 0.75 & 0.5710 & 0.4771 & 0.4485 & 0.084 \\
\hline C135 & 0.75 & $0.5562(8)$ & $0.3412(4)$ & $0.3903(3)$ & $0.0870(17)$ \\
\hline H135 & 0.75 & 0.5778 & 0.3287 & 0.4331 & 0.104 \\
\hline C136 & 0.75 & $0.5281(7)$ & $0.2693(3)$ & $0.3185(2)$ & $0.0705(14)$ \\
\hline H136 & 0.75 & 0.5288 & 0.2082 & 0.3123 & 0.085 \\
\hline
\end{tabular}




\begin{tabular}{|c|c|c|c|c|c|}
\hline C161 & 0.25 & $0.527(3)$ & $0.2909(11)$ & $0.2560(9)$ & $0.047(8)$ \\
\hline C162 & 0.25 & $0.470(2)$ & $0.3752(11)$ & $0.2857(9)$ & $0.080(10)$ \\
\hline H162 & 0.25 & 0.4104 & 0.3849 & 0.2516 & 0.096 \\
\hline C163 & 0.25 & $0.4879(19)$ & $0.4474(13)$ & $0.3578(11)$ & $0.064(6)$ \\
\hline H163 & 0.25 & 0.4353 & 0.4988 & 0.3723 & 0.076 \\
\hline C164 & 0.25 & $0.584(4)$ & $0.4419(14)$ & $0.4073(17)$ & $0.060(8)$ \\
\hline H164 & 0.25 & 0.6098 & 0.4926 & 0.4535 & 0.072 \\
\hline C165 & 0.25 & $0.6396(14)$ & $0.3595(10)$ & $0.3866(6)$ & $0.059(4)$ \\
\hline H165 & 0.25 & 0.6970 & 0.3500 & 0.4219 & 0.071 \\
\hline C166 & 0.25 & $0.6121(12)$ & $0.2896(8)$ & $0.3136(6)$ & $0.044(2)$ \\
\hline H166 & 0.25 & 0.6564 & 0.2352 & 0.3019 & 0.053 \\
\hline C141 & 0.75 & $0.3919(5)$ & $0.1015(4)$ & $0.1730(3)$ & $0.0348(16)$ \\
\hline C142 & 0.75 & $0.4547(4)$ & $0.0395(2)$ & $0.1935(2)$ & $0.0493(8)$ \\
\hline H142 & 0.75 & 0.5496 & 0.0428 & 0.1979 & 0.059 \\
\hline C143 & 0.75 & $0.3826(4)$ & $-0.0273(3)$ & $0.2078(3)$ & $0.0566(10)$ \\
\hline H143 & 0.75 & 0.4286 & -0.0686 & 0.2214 & 0.068 \\
\hline C144 & 0.75 & $0.2447(8)$ & $-0.0335(7)$ & $0.2022(6)$ & $0.0571(19)$ \\
\hline H144 & 0.75 & 0.1958 & -0.0791 & 0.2116 & 0.069 \\
\hline C145 & 0.75 & $0.1779(5)$ & $0.0272(4)$ & $0.1826(4)$ & $0.0716(16)$ \\
\hline H145 & 0.75 & 0.0829 & 0.0241 & 0.1797 & 0.086 \\
\hline C146 & 0.75 & $0.2513(3)$ & $0.0934(3)$ & $0.1672(3)$ & $0.0518(9)$ \\
\hline H146 & 0.75 & 0.2048 & 0.1336 & 0.1525 & 0.062 \\
\hline C151 & 0.25 & $0.3728(16)$ & $0.1226(9)$ & $0.1861(6)$ & $0.032(4)$ \\
\hline C152 & 0.25 & $0.3696(13)$ & $0.1257(8)$ & $0.2586(6)$ & $0.062(3)$ \\
\hline H152 & 0.25 & 0.4149 & 0.1718 & 0.3019 & 0.074 \\
\hline C153 & 0.25 & $0.2927(19)$ & $0.0542(11)$ & $0.2620(8)$ & $0.100(6)$ \\
\hline H153 & 0.25 & 0.2865 & 0.0496 & 0.3097 & 0.119 \\
\hline C154 & 0.25 & $0.226(3)$ & $-0.0098(19)$ & $0.1990(13)$ & $0.078(11)$ \\
\hline H154 & 0.25 & 0.1705 & -0.0554 & 0.2051 & 0.094 \\
\hline C155 & 0.25 & $0.2353(11)$ & $-0.0104(7)$ & $0.1273(7)$ & $0.052(3)$ \\
\hline H155 & 0.25 & 0.1910 & -0.0569 & 0.0839 & 0.063 \\
\hline C156 & 0.25 & $0.3124(11)$ & $0.0594(7)$ & $0.1199(6)$ & $0.049(2)$ \\
\hline H156 & 0.25 & 0.3222 & 0.0628 & 0.0718 & 0.059 \\
\hline $\mathrm{Ni} 2$ & 1 & $0.90195(5)$ & $0.25790(3)$ & $0.58957(2)$ & $0.04630(13)$ \\
\hline $\mathrm{Cl} 2 \mathrm{~A}$ & 0.75 & $0.73605(15)$ & $0.32026(11)$ & $0.55704(11)$ & $0.0601(4)$ \\
\hline $\mathrm{Cl} 2 \mathrm{~B}$ & 0.25 & $0.7039(3)$ & $0.2859(3)$ & $0.5542(3)$ & $0.0663(14)$ \\
\hline $\mathrm{P} 2$ & 1 & $0.99598(8)$ & $0.20846(6)$ & $0.47649(4)$ & $0.05201(19)$ \\
\hline $\mathrm{Si} 2$ & 1 & $0.67726(10)$ & $0.29851(7)$ & $0.74736(5)$ & $0.0658(3)$ \\
\hline C21 & 1 & $0.8505(3)$ & $0.2889(2)$ & $0.70681(14)$ & $0.0526(7)$ \\
\hline C22 & 1 & $0.9183(4)$ & $0.2065(2)$ & $0.67208(16)$ & $0.0614(8)$ \\
\hline H22 & 1 & 0.8836 & 0.1481 & 0.6648 & 0.074 \\
\hline $\mathrm{C} 23$ & 1 & $1.0470(4)$ & $0.2270(3)$ & $0.65020(17)$ & $0.0687(10)$ \\
\hline $\mathrm{H} 23$ & 1 & 1.1065 & 0.1841 & 0.6182 & 0.082 \\
\hline $\mathrm{C} 24$ & 1 & $1.1846(4)$ & $0.3846(4)$ & $0.6893(2)$ & $0.0951(15)$ \\
\hline $\mathrm{H} 24$ & 1 & 1.2663 & 0.3609 & 0.6664 & 0.114 \\
\hline $\mathrm{C} 25$ & 1 & $1.1720(6)$ & $0.4765(5)$ & $0.7271(3)$ & $0.124(2)$ \\
\hline H25 & 1 & 1.2475 & 0.5161 & 0.7316 & 0.149 \\
\hline C26 & 1 & $1.0544(6)$ & $0.5126(4)$ & $0.7584(3)$ & $0.122(2)$ \\
\hline H26 & 1 & 1.0503 & 0.5766 & 0.7831 & 0.146 \\
\hline $\mathrm{C} 27$ & 1 & $0.9423(4)$ & $0.4598(3)$ & $0.7553(2)$ & $0.0810(11)$ \\
\hline $\mathrm{H} 27$ & 1 & 0.8610 & 0.4864 & 0.7765 & 0.097 \\
\hline $\mathrm{C} 211$ & 1 & $0.6989(5)$ & $0.3097(3)$ & $0.8490(2)$ & $0.0888(13)$ \\
\hline $\mathrm{H} 21 \mathrm{~A}$ & 1 & 0.7410 & 0.2561 & 0.8483 & 0.133 \\
\hline $\mathrm{H} 21 \mathrm{~B}$ & 1 & 0.6112 & 0.3148 & 0.8725 & 0.133 \\
\hline $\mathrm{H} 21 \mathrm{C}$ & 1 & 0.7558 & 0.3640 & 0.8788 & 0.133 \\
\hline $\mathrm{C} 212$ & 1 & $0.5913(5)$ & $0.3994(4)$ & $0.7479(3)$ & $0.1076(17)$ \\
\hline H21D & 1 & 0.6297 & 0.4547 & 0.7896 & 0.161 \\
\hline $\mathrm{H} 21 \mathrm{E}$ & 1 & 0.4955 & 0.3929 & 0.7559 & 0.161 \\
\hline $\mathrm{H} 21 \mathrm{~F}$ & 1 & 0.6039 & 0.4032 & 0.6986 & 0.161 \\
\hline $\mathrm{C} 213$ & 1 & $0.5747(5)$ & $0.1925(4)$ & $0.6884(3)$ & $0.1103(16)$ \\
\hline $\mathrm{H} 21 \mathrm{G}$ & 1 & 0.5679 & 0.1856 & 0.6351 & 0.165 \\
\hline $\mathrm{H} 21 \mathrm{H}$ & 1 & 0.4850 & 0.1962 & 0.7093 & 0.165 \\
\hline H21I & 1 & 0.6176 & 0.1402 & 0.6898 & 0.165 \\
\hline $\mathrm{C} 231$ & 0.33 & $1.0628(16)$ & $0.2935(10)$ & 0.4439 (9) & $0.047(2)$ \\
\hline C232 & 0.33 & $1.0662(14)$ & $0.3835(10)$ & $0.4989(9)$ & $0.062(2)$ \\
\hline
\end{tabular}




\begin{tabular}{|c|c|c|c|c|c|}
\hline H232 & 0.33 & 1.0315 & 0.3974 & 0.5484 & 0.075 \\
\hline $\mathrm{C} 233$ & 0.33 & $1.1208(17)$ & $0.4526(11)$ & $0.4809(9)$ & $0.076(3)$ \\
\hline $\mathrm{H} 233$ & 0.33 & 1.1328 & 0.5136 & 0.5178 & 0.091 \\
\hline $\mathrm{C} 234$ & 0.33 & $1.156(3)$ & $0.4247(13)$ & $0.4042(9)$ & $0.088(3)$ \\
\hline H234 & 0.33 & 1.2185 & 0.4622 & 0.3918 & 0.106 \\
\hline $\mathrm{C} 235$ & 0.33 & $1.1066(19)$ & $0.3470(11)$ & $0.3465(9)$ & $0.070(3)$ \\
\hline H235 & 0.33 & 1.1078 & 0.3393 & 0.2946 & 0.084 \\
\hline $\mathrm{C} 236$ & 0.33 & $1.0521(16)$ & $0.2768(10)$ & $0.3663(8)$ & $0.059(3)$ \\
\hline $\mathrm{H} 236$ & 0.33 & 1.0113 & 0.2222 & 0.3289 & 0.071 \\
\hline C261 & 0.17 & $1.005(2)$ & $0.3032(13)$ & $0.4451(15)$ & $0.047(2)$ \\
\hline C262 & 0.17 & $0.9247(18)$ & $0.3783(11)$ & $0.4681(11)$ & $0.062(2)$ \\
\hline H262 & 0.17 & 0.8571 & 0.3817 & 0.5037 & 0.075 \\
\hline C263 & 0.17 & $0.934(2)$ & $0.4486(13)$ & $0.4438(12)$ & $0.076(3)$ \\
\hline H263 & 0.17 & 0.8763 & 0.4974 & 0.4638 & 0.091 \\
\hline C264 & 0.17 & $1.027(2)$ & $0.4460(16)$ & $0.3907(14)$ & $0.088(3)$ \\
\hline H264 & 0.17 & 1.0288 & 0.4896 & 0.3696 & 0.106 \\
\hline C265 & 0.17 & $1.119(3)$ & $0.3797(16)$ & $0.3684(14)$ & $0.070(3)$ \\
\hline H265 & 0.17 & 1.1929 & 0.3823 & 0.3383 & 0.084 \\
\hline C266 & 0.17 & $1.101(2)$ & $0.3053(13)$ & $0.3917(11)$ & 0.059 (3) \\
\hline H266 & 0.17 & 1.1560 & 0.2553 & 0.3697 & 0.071 \\
\hline $\mathrm{C} 271$ & 0.33 & $1.0369(13)$ & $0.2901(9)$ & $0.4355(10)$ & $0.047(2)$ \\
\hline $\mathrm{C} 272$ & 0.33 & $1.0997(14)$ & $0.2734(11)$ & $0.3689(8)$ & $0.062(2)$ \\
\hline H272 & 0.33 & 1.1196 & 0.2121 & 0.3393 & 0.075 \\
\hline $\mathrm{C} 273$ & 0.33 & $1.138(2)$ & $0.3337(12)$ & $0.3386(11)$ & $0.076(3)$ \\
\hline $\mathrm{H} 273$ & 0.33 & 1.1640 & 0.3136 & 0.2866 & 0.091 \\
\hline $\mathrm{C} 274$ & 0.33 & $1.139(3)$ & $0.4231(14)$ & $0.3861(10)$ & $0.088(3)$ \\
\hline H274 & 0.33 & 1.1345 & 0.4705 & 0.3697 & 0.106 \\
\hline $\mathrm{C} 275$ & 0.33 & $1.1452(17)$ & $0.4351(9)$ & 0.4609 (6) & $0.070(3)$ \\
\hline H275 & 0.33 & 1.1847 & 0.4910 & 0.4970 & 0.084 \\
\hline $\mathrm{C} 276$ & 0.33 & $1.0986(14)$ & $0.3716(9)$ & $0.4900(8)$ & $0.059(3)$ \\
\hline H276 & 0.33 & 1.1084 & 0.3833 & 0.5427 & 0.071 \\
\hline $\mathrm{C} 281$ & 0.17 & $0.996(2)$ & $0.2887(13)$ & $0.4302(13)$ & $0.047(2)$ \\
\hline $\mathrm{C} 282$ & 0.17 & $0.9034(18)$ & $0.3491(13)$ & $0.4275(11)$ & $0.062(2)$ \\
\hline H282 & 0.17 & 0.8226 & 0.3533 & 0.4542 & 0.075 \\
\hline C283 & 0.17 & $0.922(2)$ & $0.4039(14)$ & $0.3879(12)$ & 0.076 \\
\hline $\mathrm{H} 283$ & 0.17 & 0.8573 & 0.4454 & 0.3892 & 0.091 \\
\hline C284 & 0.17 & $1.037(2)$ & $0.3966(16)$ & $0.3468(14)$ & $0.088(3)$ \\
\hline H284 & 0.17 & 1.0739 & 0.4429 & 0.3335 & 0.106 \\
\hline $\mathrm{C} 285$ & 0.17 & $1.091(2)$ & $0.3148(14)$ & $0.3277(12)$ & $0.070(3)$ \\
\hline H285 & 0.17 & 1.1661 & 0.3008 & 0.2952 & 0.084 \\
\hline $\mathrm{C} 286$ & 0.17 & $1.041(3)$ & $0.2490(13)$ & $0.3537(12)$ & $0.059(3)$ \\
\hline H286 & 0.17 & 1.0391 & 0.1855 & 0.3232 & 0.071 \\
\hline C241 & 0.66 & $0.9045(7)$ & $0.1121(4)$ & $0.3981(3)$ & $0.046(3)$ \\
\hline $\mathrm{C} 242$ & 0.66 & $0.7654(4)$ & $0.1131(4)$ & $0.3989(3)$ & $0.0661(14)$ \\
\hline H242 & 0.66 & 0.7230 & 0.1592 & 0.4399 & 0.079 \\
\hline $\mathrm{C} 243$ & 0.66 & $0.6883(5)$ & $0.0465(4)$ & $0.3397(3)$ & $0.0803(18)$ \\
\hline $\mathrm{H} 243$ & 0.66 & 0.5935 & 0.0478 & 0.3408 & 0.096 \\
\hline $\mathrm{C} 244$ & 0.66 & $0.7484(13)$ & $-0.0216(7)$ & $0.2790(6)$ & $0.071(3)$ \\
\hline $\mathrm{H} 244$ & 0.66 & 0.6957 & -0.0652 & 0.2377 & 0.085 \\
\hline C245 & 0.66 & $0.8846(6)$ & $-0.0249(4)$ & $0.2800(3)$ & $0.0780(16)$ \\
\hline H245 & 0.66 & 0.9263 & -0.0734 & 0.2406 & 0.094 \\
\hline C246 & 0.66 & $0.9632(5)$ & $0.0424(4)$ & $0.3382(3)$ & $0.0727(15)$ \\
\hline H246 & 0.66 & 1.0579 & 0.0403 & 0.3368 & 0.087 \\
\hline $\mathrm{C} 251$ & 0.34 & $0.8831(17)$ & $0.1211(9)$ & $0.4021(5)$ & $0.039(4)$ \\
\hline $\mathrm{C} 252$ & 0.34 & $0.8780(9)$ & $0.1071(6)$ & $0.3251(4)$ & $0.061(2)$ \\
\hline $\mathrm{H} 252$ & 0.34 & 0.9217 & 0.1501 & 0.3102 & 0.073 \\
\hline $\mathrm{C} 253$ & 0.34 & $0.8098(12)$ & $0.0309(8)$ & $0.2696(5)$ & $0.076(3)$ \\
\hline $\mathrm{H} 253$ & 0.34 & 0.8071 & 0.0222 & 0.2173 & 0.092 \\
\hline $\mathrm{C} 254$ & 0.34 & $0.746(2)$ & $-0.0324(11)$ & $0.2914(9)$ & $0.054(5)$ \\
\hline $\mathrm{H} 254$ & 0.34 & 0.6997 & -0.0846 & 0.2539 & 0.065 \\
\hline $\mathrm{C} 255$ & 0.34 & $0.7492(9)$ & $-0.0195(6)$ & $0.3667(6)$ & $0.067(3)$ \\
\hline H255 & 0.34 & 0.7049 & -0.0625 & 0.3814 & 0.081 \\
\hline $\mathrm{C} 256$ & 0.34 & $0.8178(9)$ & $0.0569(6)$ & $0.4223(5)$ & $0.0530(19)$ \\
\hline H256 & 0.34 & 0.8198 & 0.0651 & 0.4744 & 0.064 \\
\hline $\mathrm{C} 221$ & 0.66 & $1.1580(5)$ & $0.1534(6)$ & $0.4757(6)$ & $0.063(3)$ \\
\hline
\end{tabular}




\begin{tabular}{|c|c|c|c|c|c|}
\hline$C 222$ & 0.66 & $1.2854(5)$ & $0.1892(6)$ & $0.4641(4)$ & $0.090(2)$ \\
\hline $\mathrm{H} 222$ & 0.66 & 1.2883 & 0.2422 & 0.4548 & 0.108 \\
\hline $\mathrm{C} 223$ & 0.66 & $1.4052(9)$ & $0.1494(9)$ & $0.4661(9)$ & $0.128(6)$ \\
\hline H223 & 0.66 & 1.4874 & 0.1795 & 0.4636 & 0.154 \\
\hline $\mathrm{C} 224$ & 0.66 & $1.4076(7)$ & $0.0666(8)$ & $0.4715(6)$ & $0.106(3)$ \\
\hline $\mathrm{H} 224$ & 0.66 & 1.4894 & 0.0384 & 0.4693 & 0.127 \\
\hline $\mathrm{C} 225$ & 0.66 & $1.2871(6)$ & $0.0265(6)$ & $0.4802(5)$ & $0.090(2)$ \\
\hline $\mathrm{H} 225$ & 0.66 & 1.2846 & -0.0294 & 0.4849 & 0.109 \\
\hline $\mathrm{C} 226$ & 0.66 & $1.1681(7)$ & $0.0711(5)$ & $0.4818(4)$ & $0.078(2)$ \\
\hline H226 & 0.66 & 1.0869 & 0.0422 & 0.4875 & 0.093 \\
\hline C291 & 0.34 & $1.1665(8)$ & $0.1717(7)$ & $0.4668(10)$ & $0.049(3)$ \\
\hline C292 & 0.34 & $1.2602(10)$ & $0.2422(7)$ & $0.5047(7)$ & $0.067(3)$ \\
\hline H292 & 0.34 & 1.2392 & 0.3041 & 0.5271 & 0.080 \\
\hline C293 & 0.34 & $1.3889(10)$ & $0.2129(9)$ & $0.5067(9)$ & $0.086(5)$ \\
\hline H293 & 0.34 & 1.4619 & 0.2562 & 0.5268 & 0.103 \\
\hline $\mathrm{C} 294$ & 0.34 & $1.4112(13)$ & $0.1215(9)$ & $0.4797(9)$ & $0.059(3)$ \\
\hline H294 & 0.34 & 1.4993 & 0.1025 & 0.4803 & 0.070 \\
\hline $\mathrm{C} 295$ & 0.34 & $1.3066(12)$ & $0.0585(8)$ & $0.4522(9)$ & $0.081(4)$ \\
\hline H295 & 0.34 & 1.3248 & -0.0035 & 0.4358 & 0.097 \\
\hline $\mathrm{C} 296$ & 0.34 & $1.1740(11)$ & $0.0804(8)$ & $0.4467(8)$ & $0.079(4)$ \\
\hline H296 & 0.34 & 1.0995 & 0.0379 & 0.4313 & 0.095 \\
\hline C13A & 1 & $0.5674(3)$ & $0.2807(2)$ & $0.01774(16)$ & $0.0531(7)$ \\
\hline C17A & 1 & $0.4567(2)$ & $0.33207(19)$ & 0.01499 (13) & $0.0414(5)$ \\
\hline $\mathrm{C} 23 \mathrm{~A}$ & 1 & $1.0719(3)$ & $0.3255(3)$ & $0.68543(16)$ & $0.0652(9)$ \\
\hline $\mathrm{C} 27 \mathrm{~A}$ & 1 & $0.9504(3)$ & $0.3641(2)$ & $0.71967(15)$ & $0.0570(7)$ \\
\hline
\end{tabular}

Table S2. Anisotropic displacement parameters $\left(\AA^{2}\right)$

Ni1
C11
P1
Si1
C11
C12
C13
C14
C15
C16
C17
C111
C112
C113
C121
C122
C123
C124
C125
C126
C131
C132
C133
C134
C135
C136
C161
C162
C163
C164
C165
C166
C141
C142
C143
C144

\begin{tabular}{|c|c|c|c|c|c|}
\hline$U_{11}$ & $U_{22}$ & $U_{33}$ & $U_{12}$ & $U_{13}$ & $U_{23}$ \\
\hline $0.0327(2)$ & $0.0567(3)$ & $0.0323(2)$ & $0.01083(17)$ & $0.00512(15)$ & $0.02740(19)$ \\
\hline $0.0335(3)$ & $0.0715(4)$ & $0.0417(3)$ & $0.0123(3)$ & $0.0098(2)$ & $0.0308(3)$ \\
\hline $0.0292(3)$ & $0.0552(4)$ & $0.0355(3)$ & $0.0051(2)$ & $0.0025(2)$ & $0.0294(3)$ \\
\hline $0.0427(4)$ & $0.0457(4)$ & $0.0354(3)$ & $0.0027(3)$ & $-0.0084(3)$ & $0.0200(3)$ \\
\hline $0.0437(13)$ & $0.0468(13)$ & $0.0245(10)$ & $0.0067(10)$ & $0.0025(9)$ & $0.0194(10)$ \\
\hline $0.0639(17)$ & $0.0521(15)$ & $0.0305(12)$ & $0.0190(12)$ & 0.0085 (11) & 0.0199 (11) \\
\hline $0.0597(17)$ & $0.083(2)$ & $0.0464(15)$ & $0.0377(15)$ & $0.0235(13)$ & $0.0422(15)$ \\
\hline $0.0366(16)$ & $0.162(4)$ & $0.068(2)$ & $0.005(2)$ & $0.0064(14)$ & $0.081(3)$ \\
\hline $0.049(2)$ & $0.184(5)$ & $0.073(2)$ & $-0.042(3)$ & $-0.0180(17)$ & $0.081(3)$ \\
\hline $0.072(2)$ & $0.099(3)$ & $0.063(2)$ & $-0.037(2)$ & $-0.0160(17)$ & $0.040(2)$ \\
\hline $0.0554(17)$ & $0.0671(19)$ & $0.0458(15)$ & $-0.0125(13)$ & $-0.0069(12)$ & $0.0288(14)$ \\
\hline $0.083(2)$ & $0.0661(19)$ & $0.0417(15)$ & $-0.0033(16)$ & $-0.0176(14)$ & $0.0279(14)$ \\
\hline $0.0639(19)$ & $0.0620(19)$ & $0.0618(18)$ & $0.0233(14)$ & $-0.0025(15)$ & $0.0241(16)$ \\
\hline $0.0561(18)$ & $0.072(2)$ & $0.068(2)$ & $-0.0134(14)$ & $-0.0147(15)$ & $0.0371(17)$ \\
\hline $0.0283(11)$ & $0.0472(13)$ & $0.0404(12)$ & $0.0006(9)$ & $-0.0005(9)$ & $0.0287(11)$ \\
\hline $0.0372(14)$ & $0.0562(17)$ & $0.073(2)$ & $-0.0037(12)$ & $-0.0049(13)$ & $0.0169(15)$ \\
\hline $0.0287(14)$ & $0.087(3)$ & $0.108(3)$ & $-0.0072(14)$ & $-0.0008(16)$ & $0.042(2)$ \\
\hline $0.0382(15)$ & $0.092(2)$ & $0.080(2)$ & $0.0192(15)$ & $0.0157(14)$ & $0.047(2)$ \\
\hline $0.0534(18)$ & $0.065(2)$ & $0.087(2)$ & $0.0215(15)$ & $0.0082(16)$ & $0.0267(18)$ \\
\hline $0.0376(14)$ & $0.0516(16)$ & $0.080(2)$ & $0.0048(11)$ & $-0.0039(13)$ & $0.0275(15)$ \\
\hline $0.033(4)$ & $0.057(3)$ & $0.035(3)$ & $0.0099(17)$ & $0.0077(19)$ & $0.025(2)$ \\
\hline $0.067(3)$ & $0.044(3)$ & $0.050(3)$ & $-0.010(2)$ & $-0.017(3)$ & $0.027(2)$ \\
\hline $0.075(5)$ & $0.052(3)$ & $0.075(4)$ & $-0.006(3)$ & $-0.007(3)$ & $0.020(3)$ \\
\hline $0.060(8)$ & $0.079(5)$ & $0.045(4)$ & -0.009 & $0.007(4)$ & $0.002(3)$ \\
\hline $0.122(5)$ & $0.105(5)$ & $0.035(2)$ & $-0.006(4)$ & $-0.013(3)$ & $0.031(3)$ \\
\hline $0.114(5)$ & $0.062(3)$ & $0.038(2)$ & $-0.004(3)$ & $-0.013(3)$ & $0.025(2)$ \\
\hline $0.036(12)$ & $0.064(10)$ & $0.048(10)$ & $0.018(6)$ & $0.019(5)$ & $0.029(7)$ \\
\hline $0.070(13)$ & 0.128 (19) & $0.080(15)$ & $-0.054(11)$ & $-0.047(11)$ & $0.087(14)$ \\
\hline $0.036(8)$ & $0.075(11)$ & $0.063(12)$ & $-0.015(6)$ & $-0.003(9)$ & $0.014(9)$ \\
\hline $0.036(12)$ & $0.090(16)$ & $0.043(10)$ & $-0.005(11)$ & $-0.008(6)$ & $0.016(9)$ \\
\hline $0.053(7)$ & $0.097(11)$ & $0.027(5)$ & $-0.012(7)$ & $-0.007(5)$ & $0.028(6)$ \\
\hline $0.047(6)$ & $0.049(6)$ & $0.039(6)$ & $0.003(5)$ & $0.001(5)$ & $0.021(5)$ \\
\hline $0.029(3)$ & $0.048(3)$ & $0.030(2)$ & $0.003(2)$ & $0.0076(18)$ & $0.018(2)$ \\
\hline $0.0376(18)$ & $0.061(2)$ & $0.068(2)$ & $0.0069(15)$ & $0.0077(16)$ & $0.045(2)$ \\
\hline $0.059(2)$ & $0.059(2)$ & $0.072(3)$ & $0.0087(18)$ & $0.0121(19)$ & $0.047(2)$ \\
\hline $0.060(4)$ & $0.055(4)$ & $0.068(4)$ & $-0.013(3)$ & $0.005(2)$ & $0.039(3)$ \\
\hline
\end{tabular}




\begin{tabular}{|c|c|c|c|c|c|c|}
\hline C145 & $0.038(2)$ & $0.090(4)$ & $0.113(5)$ & $-0.013(2)$ & $-0.007(3)$ & $0.070(4)$ \\
\hline C146 & $0.0374(19)$ & $0.060(2)$ & $0.075(3)$ & $-0.0062(16)$ & $-0.0071(19)$ & $0.046(2)$ \\
\hline C151 & $0.024(6)$ & $0.054(9)$ & $0.019(6)$ & $0.019(6)$ & $0.011(5)$ & $0.015(6)$ \\
\hline C152 & $0.068(8)$ & $0.072(8)$ & $0.047(6)$ & $-0.024(6)$ & $0.002(5)$ & $0.029(6)$ \\
\hline $\mathrm{C} 153$ & $0.122(14)$ & $0.123(14)$ & $0.059(8)$ & $-0.061(12)$ & $0.002(9)$ & $0.050(9)$ \\
\hline C154 & $0.062(15)$ & $0.08(2)$ & $0.096(17)$ & $-0.033(15)$ & $0.008(13)$ & $0.049(15)$ \\
\hline $\mathrm{C} 155$ & $0.039(6)$ & $0.043(6)$ & $0.067(7)$ & $-0.016(4)$ & $-0.006(5)$ & $0.017(6)$ \\
\hline C156 & $0.046(6)$ & $0.054(7)$ & $0.049(6)$ & $-0.003(5)$ & $0.003(5)$ & $0.023(5)$ \\
\hline $\mathrm{Ni} 2$ & $0.0548(3)$ & $0.0607(3)$ & $0.0262(2)$ & $0.0047(2)$ & $0.00168(18)$ & $0.0208(2)$ \\
\hline $\mathrm{Cl} 2 \mathrm{~A}$ & $0.0846(8)$ & $0.0631(10)$ & $0.0410(5)$ & $0.0171(6)$ & $-0.0072(5)$ & $0.0287(8)$ \\
\hline $\mathrm{Cl} 2 \mathrm{~B}$ & $0.115(4)$ & $0.058(3)$ & $0.0423(18)$ & $0.038(2)$ & $0.009(2)$ & $0.033(2)$ \\
\hline $\mathrm{P} 2$ & $0.0539(4)$ & $0.0711(5)$ & $0.0267(3)$ & $-0.0198(3)$ & $0.0014(3)$ & $0.0183(3)$ \\
\hline $\mathrm{Si} 2$ & $0.0726(6)$ & $0.0932(7)$ & $0.0402(4)$ & $0.0221(5)$ & $0.0164(4)$ & $0.0344(4)$ \\
\hline $\mathrm{C} 21$ & $0.0656(18)$ & $0.0733(19)$ & $0.0262(11)$ & $0.0200(14)$ & $0.0066(11)$ & $0.0262(12)$ \\
\hline $\mathrm{C} 22$ & $0.084(2)$ & $0.078(2)$ & $0.0377(14)$ & $0.0250(16)$ & $0.0112(14)$ & $0.0373(15)$ \\
\hline $\mathrm{C} 23$ & $0.072(2)$ & $0.106(3)$ & $0.0363(14)$ & 0.0418 (19) & $0.0114(14)$ & $0.0346(17)$ \\
\hline $\mathrm{C} 24$ & $0.061(2)$ & $0.160(5)$ & $0.056(2)$ & $-0.008(3)$ & $-0.0235(17)$ & $0.037(3)$ \\
\hline $\mathrm{C} 25$ & $0.103(4)$ & $0.141(5)$ & $0.104(4)$ & $-0.050(4)$ & $-0.059(3)$ & $0.034(4)$ \\
\hline $\mathrm{C} 26$ & $0.112(4)$ & $0.103(4)$ & $0.117(4)$ & $-0.031(3)$ & $-0.059(4)$ & $0.016(3)$ \\
\hline $\mathrm{C} 27$ & $0.089(3)$ & $0.080(3)$ & $0.0541(19)$ & $0.005(2)$ & $-0.0238(18)$ & $0.0082(18)$ \\
\hline $\mathrm{C} 211$ & $0.118(3)$ & $0.116(3)$ & $0.0458(18)$ & $0.037(3)$ & $0.031(2)$ & $0.043(2)$ \\
\hline $\mathrm{C} 212$ & $0.101(3)$ & $0.162(5)$ & $0.092(3)$ & $0.073(3)$ & $0.039(3)$ & $0.077(3)$ \\
\hline $\mathrm{C} 213$ & $0.099(3)$ & $0.147(5)$ & $0.081(3)$ & $-0.019(3)$ & $0.009(3)$ & $0.045(3)$ \\
\hline $\mathrm{C} 231$ & $0.025(6)$ & $0.073(3)$ & $0.040(3)$ & $-0.024(3)$ & $-0.002(3)$ & $0.023(3)$ \\
\hline $\mathrm{C} 232$ & $0.057(5)$ & $0.088(6)$ & $0.050(4)$ & $-0.006(4)$ & $0.011(4)$ & $0.037(4)$ \\
\hline C233 & $0.070(5)$ & $0.103(7)$ & $0.060(5)$ & $0.000(5)$ & $0.014(4)$ & $0.040(5)$ \\
\hline $\mathrm{C} 234$ & $0.085(6)$ & $0.120(6)$ & $0.092(8)$ & $-0.036(5)$ & $-0.011(6)$ & $0.079(6)$ \\
\hline $\mathrm{C} 235$ & $0.097(7)$ & $0.074(7)$ & $0.037(5)$ & $-0.038(6)$ & $0.008(4)$ & $0.025(5)$ \\
\hline $\mathrm{C} 236$ & $0.062(5)$ & $0.072(6)$ & $0.038(4)$ & $-0.028(4)$ & $-0.001(3)$ & $0.021(4)$ \\
\hline C261 & $0.025(6)$ & $0.073(3)$ & $0.040(3)$ & $-0.024(3)$ & $-0.002(3)$ & $0.023(3)$ \\
\hline C262 & $0.057(5)$ & $0.088(6)$ & $0.050(4)$ & $-0.006(4)$ & $0.011(4)$ & $0.037(4)$ \\
\hline C263 & $0.070(5)$ & $0.103(7)$ & $0.060(5)$ & $0.000(5)$ & $0.014(4)$ & $0.040(5)$ \\
\hline $\mathrm{C} 264$ & $0.085(6)$ & $0.120(6)$ & $0.092(8)$ & $-0.036(5)$ & $-0.011(6)$ & $0.079(6)$ \\
\hline C265 & $0.097(7)$ & $0.074(7)$ & $0.037(5)$ & $-0.038(6)$ & $0.008(4)$ & $0.025(5)$ \\
\hline C266 & $0.062(5)$ & $0.072(6)$ & $0.038(4)$ & $-0.028(4)$ & $-0.001(3)$ & $0.021(4)$ \\
\hline $\mathrm{C} 271$ & $0.025(6)$ & $0.073(3)$ & $0.040(3)$ & $-0.024(3)$ & $-0.002(3)$ & $0.023(3)$ \\
\hline $\mathrm{C} 272$ & $0.057(5)$ & $0.088(6)$ & $0.050(4)$ & $-0.006(4)$ & $0.011(4)$ & $0.037(4)$ \\
\hline $\mathrm{C} 273$ & $0.070(5)$ & $0.103(7)$ & $0.060(5)$ & $0.000(5)$ & $0.014(4)$ & $0.040(5)$ \\
\hline $\mathrm{C} 274$ & $0.085(6)$ & $0.120(6)$ & $0.092(8)$ & $-0.036(5)$ & $-0.011(6)$ & $0.079(6)$ \\
\hline $\mathrm{C} 275$ & $0.097(7)$ & $0.074(7)$ & $0.037(5)$ & $-0.038(6)$ & $0.008(4)$ & $0.025(5)$ \\
\hline $\mathrm{C} 276$ & $0.062(5)$ & $0.072(6)$ & $0.038(4)$ & $-0.028(4)$ & $-0.001(3)$ & $0.021(4)$ \\
\hline C281 & $0.025(6)$ & $0.073(3)$ & $0.040(3)$ & $-0.024(3)$ & $-0.002(3)$ & $0.023(3)$ \\
\hline C282 & $0.057(5)$ & $0.088(6)$ & $0.050(4)$ & $-0.006(4)$ & $0.011(4)$ & $0.037(4)$ \\
\hline $\mathrm{C} 283$ & $0.070(5)$ & $0.103(7)$ & $0.060(5)$ & $0.000(5)$ & 0.014 (4) & $0.040(5)$ \\
\hline $\mathrm{C} 284$ & $0.085(6)$ & $0.120(6)$ & $0.092(8)$ & $-0.036(5)$ & $-0.011(6)$ & $0.079(6)$ \\
\hline C285 & $0.097(7)$ & $0.074(7)$ & $0.037(5)$ & $-0.038(6)$ & $0.008(4)$ & $0.025(5)$ \\
\hline $\mathrm{C} 286$ & $0.062(5)$ & $0.072(6)$ & $0.038(4)$ & $-0.028(4)$ & $-0.001(3)$ & $0.021(4)$ \\
\hline C241 & 0.037 (4) & $0.065(6)$ & $0.037(4)$ & $-0.001(3)$ & $0.004(2)$ & $0.023(4)$ \\
\hline C242 & $0.039(3)$ & $0.086(4)$ & $0.047(2)$ & $-0.005(2)$ & $-0.0016(19)$ & $0.003(2)$ \\
\hline $\mathrm{C} 243$ & $0.042(3)$ & $0.105(5)$ & $0.063(3)$ & $-0.007(3)$ & $-0.006(2)$ & $0.005(3)$ \\
\hline $\mathrm{C} 244$ & $0.070(6)$ & $0.083(6)$ & $0.044(4)$ & $-0.008(5)$ & $-0.010(3)$ & $0.013(4)$ \\
\hline $\mathrm{C} 245$ & $0.065(3)$ & $0.075(4)$ & $0.059(3)$ & $0.002(3)$ & $0.001(2)$ & $-0.005(3)$ \\
\hline $\mathrm{C} 246$ & $0.047(3)$ & $0.087(4)$ & $0.054(3)$ & $-0.005(2)$ & $0.007(2)$ & $0.001(3)$ \\
\hline $\mathrm{C} 251$ & $0.036(7)$ & $0.041(7)$ & $0.027(6)$ & $0.005(5)$ & $0.001(4)$ & $0.002(5)$ \\
\hline $\mathrm{C} 252$ & $0.057(5)$ & $0.084(7)$ & $0.034(4)$ & $-0.011(4)$ & $-0.006(4)$ & $0.020(4)$ \\
\hline $\mathrm{C} 253$ & $0.076(7)$ & $0.099(9)$ & $0.042(5)$ & $-0.019(7)$ & $-0.006(5)$ & $0.019(5)$ \\
\hline $\mathrm{C} 254$ & $0.039(7)$ & $0.052(7)$ & $0.049(9)$ & $-0.001(5)$ & $-0.007(6)$ & $0.000(6)$ \\
\hline $\mathrm{C} 255$ & $0.049(5)$ & $0.053(5)$ & $0.091(8)$ & $-0.004(4)$ & $0.002(5)$ & $0.020(5)$ \\
\hline $\mathrm{C} 256$ & $0.052(5)$ & $0.057(5)$ & $0.047(4)$ & $-0.001(4)$ & $0.005(4)$ & $0.018(4)$ \\
\hline $\mathrm{C} 221$ & $0.039(3)$ & $0.130(7)$ & $0.027(3)$ & $-0.041(3)$ & $-0.001(2)$ & $0.045(4)$ \\
\hline$C 222$ & $0.045(3)$ & $0.147(7)$ & $0.106(6)$ & $-0.026(4)$ & $-0.013(3)$ & $0.083(5)$ \\
\hline $\mathrm{C} 223$ & $0.037(4)$ & $0.223(16)$ & $0.189(15)$ & $-0.020(7)$ & $-0.016(6)$ & $0.151(12)$ \\
\hline $\mathrm{C} 224$ & $0.045(4)$ & $0.196(11)$ & $0.112(7)$ & $0.024(6)$ & $0.006(4)$ & $0.097(9)$ \\
\hline $\mathrm{C} 225$ & $0.054(3)$ & $0.136(7)$ & $0.095(5)$ & $0.024(4)$ & $0.010(3)$ & $0.059(5)$ \\
\hline $\mathrm{C} 226$ & $0.047(3)$ & $0.114(5)$ & $0.068(4)$ & $0.008(3)$ & $0.015(3)$ & $0.034(4)$ \\
\hline
\end{tabular}




$\begin{array}{llllrrl}\mathrm{C} 291 & 0.051(7) & 0.080(7) & 0.024(5) & -0.022(5) & 0.011(4) & 0.033(6) \\ \mathrm{C} 292 & 0.049(6) & 0.067(7) & 0.077(8) & -0.015(5) & -0.021(5) & 0.025(6) \\ \mathrm{C} 293 & 0.042(6) & 0.134(13) & 0.092(10) & -0.033(7) & -0.029(6) & 0.062(10) \\ \mathrm{C} 294 & 0.037(5) & 0.071(9) & 0.078(8) & 0.021(6) & 0.010(5) & 0.038(8) \\ \mathrm{C} 295 & 0.071(9) & 0.076(8) & 0.091(10) & 0.028(6) & -0.002(7) & 0.029(7) \\ \mathrm{C} 296 & 0.041(6) & 0.107(11) & 0.056(8) & 0.005(6) & -0.005(6) & 0.002(7) \\ \mathrm{C} 13 \mathrm{~A} & 0.0379(13) & 0.100(2) & 0.0418(14) & 0.0148(13) & 0.0120(11) & 0.0486(15) \\ \mathrm{C} 17 \mathrm{~A} & 0.0368(12) & 0.0654(16) & 0.0303(11) & 0.0020(11) & 0.0031(9) & 0.0283(11) \\ \mathrm{C} 23 \mathrm{~A} & 0.0626(19) & 0.103(3) & 0.0312(13) & 0.0112(17) & -0.0121(12) & 0.0284(16) \\ \mathrm{C} 27 \mathrm{~A} & 0.0654(19) & 0.076(2) & 0.0282(12) & 0.0116(15) & -0.0089(12) & 0.0194(13)\end{array}$

Ni1-C13
Ni1-C12
Ni1-C11
Ni1-C11
Ni1-P1
Ni1-C13a
Ni1-C17a
P1-C151
P1-C131
P1-C121
P1-C141
P1-C161
Si1-C112
Si1-C113
Si1-C111
Si1-C11
C11-C12
C11-C17a
C12-C13
C12-H12
C13-C13a
C13-H13
C14-C15
C14-C13a
C14-H14
C15-C16
C15-H15
C16-C17
C16-H16
C17-C17a
C17-H17
C111-H11d
C111-H11e
C111-H11f
C112-H11a
C112-H11b
C112-H11c
C113-H11g
C113-H11h
C113-H11i
C121-C122
C121-C126
C122-C123
C122-H122
C123-C124
C123-H123
C124-C125
C124-H124
C125-C126
C125-H125

Table $S 3$. Geometric parameters $\left(\AA,^{\circ}\right)$

\begin{tabular}{|c|c|c|}
\hline $2.052(3)$ & $\mathrm{C} 126-\mathrm{H} 126$ & 0.94 \\
\hline $2.070(2)$ & $\mathrm{C} 131-\mathrm{C} 132$ & $1.371(5)$ \\
\hline $2.128(2)$ & $\mathrm{C} 131-\mathrm{C} 136$ & $1.371(5)$ \\
\hline $2.1828(7)$ & $\mathrm{C} 132-\mathrm{C} 133$ & $1.379(6)$ \\
\hline $2.1886(7)$ & $\mathrm{C} 132-\mathrm{H} 132$ & 0.94 \\
\hline $2.323(3)$ & $\mathrm{C} 133-\mathrm{C} 134$ & $1.369(7)$ \\
\hline $2.335(2)$ & C133-H133 & 0.94 \\
\hline $1.796(8)$ & $\mathrm{C} 134-\mathrm{C} 135$ & $1.353(9)$ \\
\hline $1.819(4)$ & $\mathrm{C} 134-\mathrm{H} 134$ & 0.94 \\
\hline $1.822(2)$ & $\mathrm{C} 135-\mathrm{C} 136$ & $1.387(6)$ \\
\hline $1.851(4)$ & $\mathrm{C} 135-\mathrm{H} 135$ & 0.94 \\
\hline $1.854(8)$ & $\mathrm{C} 136-\mathrm{H} 136$ & 0.94 \\
\hline $1.854(3)$ & $\mathrm{C} 161-\mathrm{C} 162$ & $1.381(7)$ \\
\hline $1.860(3)$ & $\mathrm{C} 161-\mathrm{C} 166$ & $1.383(7)$ \\
\hline $1.873(3)$ & $\mathrm{C} 162-\mathrm{C} 163$ & $1.380(8)$ \\
\hline $1.877(2)$ & C162-H162 & 0.94 \\
\hline $1.423(3)$ & $\mathrm{C} 163-\mathrm{C} 164$ & $1.360(18)$ \\
\hline $1.471(3)$ & C163-H163 & 0.94 \\
\hline $1.402(4)$ & $\mathrm{C} 164-\mathrm{C} 165$ & $1.362(17)$ \\
\hline 0.94 & $\mathrm{C} 164-\mathrm{H} 164$ & 0.94 \\
\hline $1.451(5)$ & $\mathrm{C} 165-\mathrm{C} 166$ & $1.388(13)$ \\
\hline 0.94 & C165-H165 & 0.94 \\
\hline $1.367(6)$ & C166-H166 & 0.94 \\
\hline $1.396(5)$ & $\mathrm{C} 141-\mathrm{C} 142$ & $1.382(5)$ \\
\hline 0.94 & $\mathrm{C} 141-\mathrm{C} 146$ & $1.392(5)$ \\
\hline $1.393(6)$ & $\mathrm{C} 142-\mathrm{C} 143$ & $1.384(4)$ \\
\hline 0.94 & $\mathrm{C} 142-\mathrm{H} 142$ & 0.94 \\
\hline $1.383(4)$ & $\mathrm{C} 143-\mathrm{C} 144$ & $1.365(7)$ \\
\hline 0.94 & $\mathrm{C} 143-\mathrm{H} 143$ & 0.94 \\
\hline $1.392(4)$ & $\mathrm{C} 144-\mathrm{C} 145$ & $1.373(9)$ \\
\hline 0.94 & C144-H144 & 0.94 \\
\hline 0.97 & $\mathrm{C} 145-\mathrm{C} 146$ & $1.394(6)$ \\
\hline 0.97 & C145-H145 & 0.94 \\
\hline 0.97 & $\mathrm{C} 146-\mathrm{H} 146$ & 0.94 \\
\hline 0.97 & $\mathrm{C} 151-\mathrm{C} 152$ & $1.355(7)$ \\
\hline 0.97 & $\mathrm{C} 151-\mathrm{C} 156$ & $1.357(7)$ \\
\hline 0.97 & $\mathrm{C} 152-\mathrm{C} 153$ & $1.376(7)$ \\
\hline 0.97 & $\mathrm{C} 152-\mathrm{H} 152$ & 0.94 \\
\hline 0.97 & $\mathrm{C} 153-\mathrm{C} 154$ & $1.354(18)$ \\
\hline 0.97 & C153-H153 & 0.94 \\
\hline $1.378(3)$ & $\mathrm{C} 154-\mathrm{C} 155$ & $1.360(18)$ \\
\hline $1.390(4)$ & C154-H154 & 0.94 \\
\hline $1.386(4)$ & $\mathrm{C} 155-\mathrm{C} 156$ & $1.386(12)$ \\
\hline 0.94 & C155-H155 & 0.94 \\
\hline $1.360(5)$ & C156-H156 & 0.94 \\
\hline 0.94 & $\mathrm{Ni} 2-\mathrm{C} 23$ & $2.038(3)$ \\
\hline $1.367(5)$ & $\mathrm{Ni} 2-\mathrm{C} 22$ & $2.060(3)$ \\
\hline 0.94 & $\mathrm{Ni2}-\mathrm{C} 21$ & $2.135(2)$ \\
\hline $1.382(4)$ & $\mathrm{Ni} 2-\mathrm{Cl} 2 \mathrm{a}$ & $2.1770(11)$ \\
\hline 0.94 & $\mathrm{Ni} 2-\mathrm{P} 2$ & $2.1819(8)$ \\
\hline
\end{tabular}




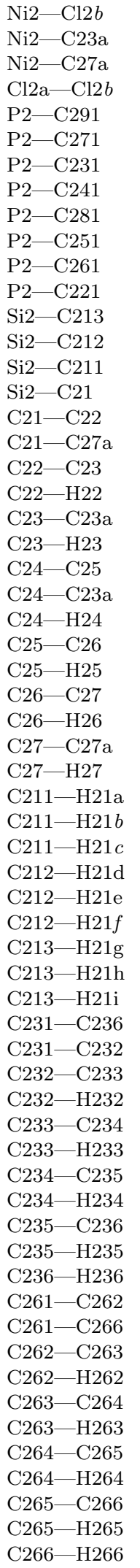

$2.195(2)$
$2.339(3)$
$2.374(3)$
$0.608(4)$
$1.800(8)$
$1.817(6)$
$1.823(7)$
$1.837(5)$
$1.840(8)$
$1.845(7)$
$1.853(8)$
$1.865(6)$
$1.854(5)$
$1.864(4)$
$1.868(3)$
$1.871(3)$
$1.415(4)$
$1.466(5)$
$1.410(5)$
0.94
$1.449(5)$
0.94
$1.361(8)$
$1.415(6)$
0.94
$1.353(8)$
0.94
$1.353(7)$
0.94
$1.408(5)$
0.94
0.97
0.97
0.97
0.97
0.97
0.97
0.97
0.97
0.97
$1.383(8)$
$1.386(8)$
$1.381(8)$
0.94
$1.385(9)$
0.94
$1.343(18)$
0.94
$1.417(12)$
0.94
0.94
$1.394(8)$
$1.396(8)$
$1.387(8)$
0.94
$1.360(17)$
0.94
$1.36(2)$
0.94
$1.444(16)$
0.94
0.94

C271-C272

C271-C276

$\mathrm{C} 272$ - 2273

C272- 2272

C273-C274

C273- $\mathrm{H} 273$

C274-C275

C274-H274

C275-C276

C275-H275

C276- $\mathrm{H} 276$

C281-C282

C281-C286

C282-C283

C282-H282

C283-C284

$\mathrm{C} 283-\mathrm{H} 283$

$\mathrm{C} 284-\mathrm{C} 285$

$\mathrm{C} 284-\mathrm{H} 284$

$\mathrm{C} 285-\mathrm{C} 286$

C285-H285

C286-H286

C241-C246

C241-C242

C242-C243

C242- $\mathrm{H} 242$

C243-C244

C243-H243

C244-C245

C244-H244

C245-C246

C245- H245

C246-H246

$\mathrm{C} 251-\mathrm{C} 256$

C251-C252

$\mathrm{C} 252-\mathrm{C} 253$

C252-H 252

C253-C254

C253-H253

$\mathrm{C} 254-\mathrm{C} 255$

C254-H254

C255-C256

C255-H255

C256-H256

C221-C226

$\mathrm{C} 221-\mathrm{C} 222$

C222-C223

C222- $\mathrm{H} 222$

C223-C224

$\mathrm{C} 223-\mathrm{H} 223$

$\mathrm{C} 224-\mathrm{C} 225$

C224- $\mathrm{H} 224$

C225-C226

$\mathrm{C} 225-\mathrm{H} 225$

$\mathrm{C} 226$ - $\mathrm{H} 226$

C291-C296

C291-C292

$\mathrm{C} 292-\mathrm{C} 293$

C292-H292

C293-C294

C293-H293

C294-C295

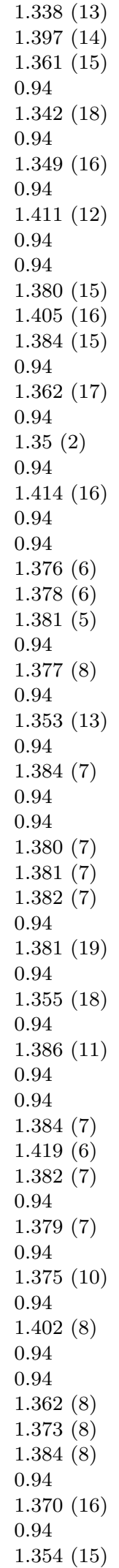




\begin{tabular}{|c|c|}
\hline С294-H294 & 0.94 \\
\hline $\mathrm{C} 295-\mathrm{C} 296$ & $1.390(13)$ \\
\hline $\mathrm{C} 295-\mathrm{H} 295$ & 0.94 \\
\hline $\mathrm{C} 13-\mathrm{NI} 1-\mathrm{C} 12$ & $39.77(12)$ \\
\hline $\mathrm{C} 13-\mathrm{NI} 1-\mathrm{C} 11$ & $66.9(1)$ \\
\hline $\mathrm{C} 12-\mathrm{NI} 1-\mathrm{C} 11$ & $39.60(9)$ \\
\hline C13-NI1-CL1 & $162.96(8)$ \\
\hline C12-NI1-CL1 & $124.76(8)$ \\
\hline C11-NI1-CL1 & $96.23(7)$ \\
\hline C13-NI1-P1 & $100.38(8)$ \\
\hline $\mathrm{C} 12-\mathrm{NI} 1-\mathrm{P} 1$ & $131.84(7)$ \\
\hline $\mathrm{C} 11-\mathrm{NI} 1-\mathrm{P} 1$ & $166.65(7)$ \\
\hline CL1-NI1-P1 & $96.61(3)$ \\
\hline $\mathrm{C} 13-\mathrm{NI} 1-\mathrm{C} 13 \mathrm{~A}$ & $38.10(12)$ \\
\hline $\mathrm{C} 12-\mathrm{NI} 1-\mathrm{C} 13 \mathrm{~A}$ & $63.15(12)$ \\
\hline $\mathrm{C} 11-\mathrm{NI} 1-\mathrm{C} 13 \mathrm{~A}$ & $63.32(9)$ \\
\hline $\mathrm{CL} 1-\mathrm{NI} 1-\mathrm{C} 13 \mathrm{~A}$ & $137.25(9)$ \\
\hline $\mathrm{P} 1-\mathrm{NI} 1-\mathrm{C} 13 \mathrm{~A}$ & $104.34(7)$ \\
\hline $\mathrm{C} 13-\mathrm{NI} 1-\mathrm{C} 17 \mathrm{~A}$ & $62.99(11)$ \\
\hline $\mathrm{C} 12-\mathrm{NI} 1-\mathrm{C} 17 \mathrm{~A}$ & $62.88(10)$ \\
\hline $\mathrm{C} 11-\mathrm{NI} 1-\mathrm{C} 17 \mathrm{~A}$ & $38.12(9)$ \\
\hline CL1-NI1-C17A & $105.58(7)$ \\
\hline $\mathrm{P} 1-\mathrm{NI} 1-\mathrm{C} 17 \mathrm{~A}$ & $133.40(6)$ \\
\hline $\mathrm{C} 13 \mathrm{~A}-\mathrm{NI} 1-\mathrm{C} 17 \mathrm{~A}$ & $35.41(9)$ \\
\hline C151-P1-C131 & $96.5(5)$ \\
\hline $\mathrm{C} 151-\mathrm{P} 1-\mathrm{C} 121$ & $111.8(6)$ \\
\hline $\mathrm{C} 131-\mathrm{P} 1-\mathrm{C} 121$ & $104.5(4)$ \\
\hline $\mathrm{C} 151-\mathrm{P} 1-\mathrm{C} 141$ & $12.0(7)$ \\
\hline C131-P1—C141 & $105.4(4)$ \\
\hline $\mathrm{C} 121-\mathrm{P} 1-\mathrm{C} 141$ & $101.28(18)$ \\
\hline $\mathrm{C} 151-\mathrm{P} 1-\mathrm{C} 161$ & $101.90(11)$ \\
\hline $\mathrm{C} 131-\mathrm{P} 1-\mathrm{C} 161$ & $8.50(13)$ \\
\hline $\mathrm{C} 121-\mathrm{P} 1-\mathrm{C} 161$ & $96.3(8)$ \\
\hline C141-P1-C161 & $109.7(1)$ \\
\hline C151-P1-NI1 & $113.1(5)$ \\
\hline C131-P1-NI1 & $114.35(17)$ \\
\hline $\mathrm{C} 121-\mathrm{P} 1-\mathrm{NI} 1$ & $114.90(7)$ \\
\hline $\mathrm{C} 141-\mathrm{P} 1-\mathrm{NI} 1$ & $114.98(15)$ \\
\hline C161-P1—NI1 & $117.1(5)$ \\
\hline $\mathrm{C} 112-\mathrm{SI} 1-\mathrm{C} 113$ & $110.63(17)$ \\
\hline C112-SI1-C111 & $108.37(15)$ \\
\hline C113-SI1-C111 & $109.53(15)$ \\
\hline C112-SI1—C11 & $113.38(13)$ \\
\hline C113-SI1-C11 & $108.10(13)$ \\
\hline C111-SI1-C11 & $106.71(13)$ \\
\hline $\mathrm{C} 12-\mathrm{C} 11-\mathrm{C} 17 \mathrm{~A}$ & $105.9(2)$ \\
\hline C12-C11-SI1 & $123.72(19)$ \\
\hline $\mathrm{C} 17 \mathrm{~A}-\mathrm{C} 11-\mathrm{SI} 1$ & $129.34(18)$ \\
\hline $\mathrm{C} 12-\mathrm{C} 11-\mathrm{NI} 1$ & 68.01 \\
\hline $\mathrm{C} 17 \mathrm{~A}-\mathrm{C} 11-\mathrm{NI} 1$ & $78.60(13)$ \\
\hline $\mathrm{SI} 1-\mathrm{C} 11-\mathrm{NI} 1$ & $126.85(12)$ \\
\hline $\mathrm{C} 13-\mathrm{C} 12-\mathrm{C} 11$ & $109.4(3)$ \\
\hline $\mathrm{C} 13-\mathrm{C} 12-\mathrm{NI} 1$ & $69.44(15)$ \\
\hline $\mathrm{C} 11-\mathrm{C} 12-\mathrm{NI} 1$ & $72.39(14)$ \\
\hline $\mathrm{C} 13-\mathrm{C} 12-\mathrm{H} 12$ & 125.3 \\
\hline $\mathrm{C} 11-\mathrm{C} 12-\mathrm{H} 12$ & 125.3 \\
\hline $\mathrm{NI} 1-\mathrm{C} 12-\mathrm{H} 12$ & 124.4 \\
\hline $\mathrm{C} 12-\mathrm{C} 13-\mathrm{C} 13 \mathrm{~A}$ & $108.1(2)$ \\
\hline $\mathrm{C} 12-\mathrm{C} 13-\mathrm{NI} 1$ & $70.78(15)$ \\
\hline $\mathrm{C} 13 \mathrm{~A}-\mathrm{C} 13-\mathrm{NI} 1$ & $81.11(16)$ \\
\hline $\mathrm{C} 12-\mathrm{C} 13-\mathrm{H} 13$ & 125.9 \\
\hline $\mathrm{C} 13 \mathrm{~A}-\mathrm{C} 13-\mathrm{H} 13$ & 125.9 \\
\hline
\end{tabular}

\begin{tabular}{|c|c|}
\hline $\mathrm{C} 296-\mathrm{H} 296$ & 0.94 \\
\hline $\mathrm{C} 13 \mathrm{a}-\mathrm{C} 17 \mathrm{a}$ & $1.417(4)$ \\
\hline $\mathrm{C} 23 \mathrm{a}-\mathrm{C} 27 \mathrm{a}$ & $1.412(5)$ \\
\hline NI1-C13-H13 & 114.3 \\
\hline $\mathrm{C} 15-\mathrm{C} 14-\mathrm{C} 13 \mathrm{~A}$ & $118.2(3)$ \\
\hline $\mathrm{C} 15-\mathrm{C} 14-\mathrm{H} 14$ & 120.9 \\
\hline $\mathrm{C} 13 \mathrm{~A}-\mathrm{C} 14-\mathrm{H} 14$ & 120.9 \\
\hline $\mathrm{C} 14-\mathrm{C} 15-\mathrm{C} 16$ & $121.7(3)$ \\
\hline $\mathrm{C} 14-\mathrm{C} 15-\mathrm{H} 15$ & 119.1 \\
\hline $\mathrm{C} 16-\mathrm{C} 15-\mathrm{H} 15$ & 119.1 \\
\hline $\mathrm{C} 17-\mathrm{C} 16-\mathrm{C} 15$ & $120.8(4)$ \\
\hline $\mathrm{C} 17-\mathrm{C} 16-\mathrm{H} 16$ & 119.6 \\
\hline $\mathrm{C} 15-\mathrm{C} 16-\mathrm{H} 16$ & 119.6 \\
\hline $\mathrm{C} 16-\mathrm{C} 17-\mathrm{C} 17 \mathrm{~A}$ & 118.8 \\
\hline $\mathrm{C} 16-\mathrm{C} 17-\mathrm{H} 17$ & 120.6 \\
\hline $\mathrm{C} 17 \mathrm{~A}-\mathrm{C} 17-\mathrm{H} 17$ & 120.6 \\
\hline SI1-C111-H11D & 109.5 \\
\hline SI1-C111-H11E & 109.5 \\
\hline $\mathrm{H} 11 \mathrm{D}-\mathrm{C} 111-\mathrm{H} 11 \mathrm{E}$ & 109.5 \\
\hline SI1-C111-H11F & 109.5 \\
\hline $\mathrm{H} 11 \mathrm{D}-\mathrm{C} 111-\mathrm{H} 11 \mathrm{~F}$ & 109.5 \\
\hline $\mathrm{H} 11 \mathrm{E}-\mathrm{C} 111-\mathrm{H} 11 \mathrm{~F}$ & 109.5 \\
\hline SI1-C112-H11A & 109.5 \\
\hline SI1-C112-H11B & 109.5 \\
\hline $\mathrm{H} 11 \mathrm{~A}-\mathrm{C} 112-\mathrm{H} 11 \mathrm{~B}$ & 109.5 \\
\hline $\mathrm{SI} 1-\mathrm{C} 112-\mathrm{H} 11 \mathrm{C}$ & 109.5 \\
\hline $\mathrm{H} 11 \mathrm{~A}-\mathrm{C} 112-\mathrm{H} 11 \mathrm{C}$ & 109.5 \\
\hline $\mathrm{H} 11 \mathrm{~B}-\mathrm{C} 112-\mathrm{H} 11 \mathrm{C}$ & 109.5 \\
\hline SI1-C113-H11G & 109.5 \\
\hline $\mathrm{SI} 1-\mathrm{C} 113-\mathrm{H} 11 \mathrm{H}$ & 109.5 \\
\hline $\mathrm{H} 11 \mathrm{G}-\mathrm{C} 113-\mathrm{H} 11 \mathrm{H}$ & 109.5 \\
\hline SI1-C113-H11I & 109.5 \\
\hline $\mathrm{H} 11 \mathrm{G}-\mathrm{C} 113-\mathrm{H} 11 \mathrm{I}$ & 109.5 \\
\hline $\mathrm{H} 11 \mathrm{H}-\mathrm{C} 113-\mathrm{H} 11 \mathrm{I}$ & 109.5 \\
\hline $\mathrm{C} 122-\mathrm{C} 121-\mathrm{C} 126$ & $117.7(2)$ \\
\hline $\mathrm{C} 122-\mathrm{C} 121-\mathrm{P} 1$ & $121.7(2)$ \\
\hline $\mathrm{C} 126-\mathrm{C} 121-\mathrm{P} 1$ & $120.25(18)$ \\
\hline $\mathrm{C} 121-\mathrm{C} 122-\mathrm{C} 123$ & $120.6(3)$ \\
\hline $\mathrm{C} 121-\mathrm{C} 122-\mathrm{H} 122$ & 119.7 \\
\hline $\mathrm{C} 123-\mathrm{C} 122-\mathrm{H} 122$ & 119.7 \\
\hline $\mathrm{C} 124-\mathrm{C} 123-\mathrm{C} 122$ & $120.7(3)$ \\
\hline $\mathrm{C} 124-\mathrm{C} 123-\mathrm{H} 123$ & 119.6 \\
\hline $\mathrm{C} 122-\mathrm{C} 123-\mathrm{H} 123$ & 119.6 \\
\hline $\mathrm{C} 123-\mathrm{C} 124-\mathrm{C} 125$ & $119.9(3)$ \\
\hline $\mathrm{C} 123-\mathrm{C} 124-\mathrm{H} 124$ & 120.1 \\
\hline $\mathrm{C} 125-\mathrm{C} 124-\mathrm{H} 124$ & 120.1 \\
\hline $\mathrm{C} 124-\mathrm{C} 125-\mathrm{C} 126$ & $119.7(3)$ \\
\hline $\mathrm{C} 124-\mathrm{C} 125-\mathrm{H} 125$ & 120.1 \\
\hline $\mathrm{C} 126-\mathrm{C} 125-\mathrm{H} 125$ & 120.1 \\
\hline $\mathrm{C} 125-\mathrm{C} 126-\mathrm{C} 121$ & $121.3(3)$ \\
\hline $\mathrm{C} 125-\mathrm{C} 126-\mathrm{H} 126$ & 119.4 \\
\hline $\mathrm{C} 121-\mathrm{C} 126-\mathrm{H} 126$ & 119.4 \\
\hline $\mathrm{C} 132-\mathrm{C} 131-\mathrm{C} 136$ & $121.9(4)$ \\
\hline $\mathrm{C} 132-\mathrm{C} 131-\mathrm{P} 1$ & $118.7(4)$ \\
\hline $\mathrm{C} 136-\mathrm{C} 131-\mathrm{P} 1$ & $119.3(4)$ \\
\hline $\mathrm{C} 131-\mathrm{C} 132-\mathrm{C} 133$ & $118.3(5)$ \\
\hline $\mathrm{C} 131-\mathrm{C} 132-\mathrm{H} 132$ & 120.9 \\
\hline $\mathrm{C} 133-\mathrm{C} 132-\mathrm{H} 132$ & 120.9 \\
\hline $\mathrm{C} 134-\mathrm{C} 133-\mathrm{C} 132$ & $120.7(6)$ \\
\hline C134-C133-H133 & 119.7 \\
\hline $\mathrm{C} 132-\mathrm{C} 133-\mathrm{H} 133$ & 119.7 \\
\hline $\mathrm{C} 135-\mathrm{C} 134-\mathrm{C} 133$ & $120.0(7)$ \\
\hline
\end{tabular}




\begin{tabular}{|c|c|}
\hline С135-C134-Н134 & 120 \\
\hline $\mathrm{C} 133-\mathrm{C} 134-\mathrm{H} 134$ & 120 \\
\hline $\mathrm{C} 134-\mathrm{C} 135-\mathrm{C} 136$ & $120.9(6)$ \\
\hline $\mathrm{C} 134-\mathrm{C} 135-\mathrm{H} 135$ & 119.6 \\
\hline $\mathrm{C} 136-\mathrm{C} 135-\mathrm{H} 135$ & 119.6 \\
\hline $\mathrm{C} 131-\mathrm{C} 136-\mathrm{C} 135$ & $118.2(4)$ \\
\hline $\mathrm{C} 131-\mathrm{C} 136-\mathrm{H} 136$ & 120.9 \\
\hline $\mathrm{C} 135-\mathrm{C} 136-\mathrm{H} 136$ & 120.9 \\
\hline $\mathrm{C} 162-\mathrm{C} 161-\mathrm{C} 166$ & $106.7(1)$ \\
\hline $\mathrm{C} 162-\mathrm{C} 161-\mathrm{P} 1$ & $124.20(11)$ \\
\hline $\mathrm{C} 166-\mathrm{C} 161-\mathrm{P} 1$ & $128.9(1)$ \\
\hline $\mathrm{C} 163-\mathrm{C} 162-\mathrm{C} 161$ & $130.00(15)$ \\
\hline $\mathrm{C} 163-\mathrm{C} 162-\mathrm{H} 162$ & 115 \\
\hline $\mathrm{C} 161-\mathrm{C} 162-\mathrm{H} 162$ & 115 \\
\hline $\mathrm{C} 164-\mathrm{C} 163-\mathrm{C} 162$ & $118.10(18)$ \\
\hline C164-C163-H163 & 121 \\
\hline $\mathrm{C} 162-\mathrm{C} 163-\mathrm{H} 163$ & 121 \\
\hline $\mathrm{C} 163-\mathrm{C} 164-\mathrm{C} 165$ & $116.80(18)$ \\
\hline $\mathrm{C} 163-\mathrm{C} 164-\mathrm{H} 164$ & 121.6 \\
\hline $\mathrm{C} 165-\mathrm{C} 164-\mathrm{H} 164$ & 121.6 \\
\hline $\mathrm{C} 164-\mathrm{C} 165-\mathrm{C} 166$ & $120.20(13)$ \\
\hline $\mathrm{C} 164-\mathrm{C} 165-\mathrm{H} 165$ & 119.9 \\
\hline $\mathrm{C} 166-\mathrm{C} 165-\mathrm{H} 165$ & 119.9 \\
\hline $\mathrm{C} 161-\mathrm{C} 166-\mathrm{C} 165$ & $127.4(1)$ \\
\hline $\mathrm{C} 161-\mathrm{C} 166-\mathrm{H} 166$ & 116.3 \\
\hline $\mathrm{C} 165-\mathrm{C} 166-\mathrm{H} 166$ & 116.3 \\
\hline $\mathrm{C} 142-\mathrm{C} 141-\mathrm{C} 146$ & $116.6(3)$ \\
\hline $\mathrm{C} 142-\mathrm{C} 141-\mathrm{P} 1$ & $124.6(3)$ \\
\hline $\mathrm{C} 146-\mathrm{C} 141-\mathrm{P} 1$ & $118.7(3)$ \\
\hline $\mathrm{C} 141-\mathrm{C} 142-\mathrm{C} 143$ & $122.1(3)$ \\
\hline C141-C142-Н142 & 118.9 \\
\hline $\mathrm{C} 143-\mathrm{C} 142-\mathrm{H} 142$ & 118.9 \\
\hline $\mathrm{C} 144-\mathrm{C} 143-\mathrm{C} 142$ & $120.2(5)$ \\
\hline $\mathrm{C} 144-\mathrm{C} 143-\mathrm{H} 143$ & 119.9 \\
\hline $\mathrm{C} 142-\mathrm{C} 143-\mathrm{H} 143$ & 119.9 \\
\hline $\mathrm{C} 143-\mathrm{C} 144-\mathrm{C} 145$ & $119.7(7)$ \\
\hline $\mathrm{C} 143-\mathrm{C} 144-\mathrm{H} 144$ & 120.1 \\
\hline $\mathrm{C} 145-\mathrm{C} 144-\mathrm{H} 144$ & 120.1 \\
\hline $\mathrm{C} 144-\mathrm{C} 145-\mathrm{C} 146$ & $119.8(6)$ \\
\hline C144-C145-Н145 & 120.1 \\
\hline $\mathrm{C} 146-\mathrm{C} 145-\mathrm{H} 145$ & 120.1 \\
\hline $\mathrm{C} 141-\mathrm{C} 146-\mathrm{C} 145$ & $121.6(4)$ \\
\hline $\mathrm{C} 141-\mathrm{C} 146-\mathrm{H} 146$ & 119.2 \\
\hline $\mathrm{C} 145-\mathrm{C} 146-\mathrm{H} 146$ & 119.2 \\
\hline $\mathrm{C} 152-\mathrm{C} 151-\mathrm{C} 156$ & $128.4(9)$ \\
\hline $\mathrm{C} 152-\mathrm{C} 151-\mathrm{P} 1$ & $123.5(7)$ \\
\hline $\mathrm{C} 156-\mathrm{C} 151-\mathrm{P} 1$ & $107.6(7)$ \\
\hline $\mathrm{C} 151-\mathrm{C} 152-\mathrm{C} 153$ & $112.6(9)$ \\
\hline $\mathrm{C} 151-\mathrm{C} 152-\mathrm{H} 152$ & 123.7 \\
\hline $\mathrm{C} 153-\mathrm{C} 152-\mathrm{H} 152$ & 123.7 \\
\hline $\mathrm{C} 154-\mathrm{C} 153-\mathrm{C} 152$ & $122.40(12)$ \\
\hline $\mathrm{C} 154-\mathrm{C} 153-\mathrm{H} 153$ & 118.8 \\
\hline $\mathrm{C} 152-\mathrm{C} 153-\mathrm{H} 153$ & 118.8 \\
\hline $\mathrm{C} 153-\mathrm{C} 154-\mathrm{C} 155$ & $122.10(14)$ \\
\hline $\mathrm{C} 153-\mathrm{C} 154-\mathrm{H} 154$ & 118.9 \\
\hline $\mathrm{C} 155-\mathrm{C} 154-\mathrm{H} 154$ & 118.9 \\
\hline $\mathrm{C} 154-\mathrm{C} 155-\mathrm{C} 156$ & $118.20(11)$ \\
\hline $\mathrm{C} 154-\mathrm{C} 155-\mathrm{H} 155$ & 120.9 \\
\hline $\mathrm{C} 156-\mathrm{C} 155-\mathrm{H} 155$ & 120.9 \\
\hline $\mathrm{C} 151-\mathrm{C} 156-\mathrm{C} 155$ & $116.1(9)$ \\
\hline C151-C156-H156 & 122 \\
\hline $\mathrm{C} 155-\mathrm{C} 156-\mathrm{H} 156$ & 122 \\
\hline
\end{tabular}

\begin{tabular}{|c|c|}
\hline $\mathrm{C} 23-\mathrm{NI} 2-\mathrm{C} 22$ & $40.25(14)$ \\
\hline $\mathrm{C} 23-\mathrm{NI} 2-\mathrm{C} 21$ & $66.49(11)$ \\
\hline $\mathrm{C} 22-\mathrm{NI} 2-\mathrm{C} 21$ & $39.38(11)$ \\
\hline $\mathrm{C} 23-\mathrm{NI} 2-\mathrm{CL} 2 \mathrm{~A}$ & $163.87(11)$ \\
\hline $\mathrm{C} 22-\mathrm{NI} 2-\mathrm{CL} 2 \mathrm{~A}$ & $131.65(11)$ \\
\hline $\mathrm{C} 21-\mathrm{NI} 2-\mathrm{CL} 2 \mathrm{~A}$ & $99.32(9)$ \\
\hline $\mathrm{C} 23-\mathrm{NI} 2-\mathrm{P} 2$ & $99.14(9)$ \\
\hline $\mathrm{C} 22-\mathrm{NI} 2-\mathrm{P} 2$ & $127.33(9)$ \\
\hline $\mathrm{C} 21-\mathrm{NI} 2-\mathrm{P} 2$ & $165.40(8)$ \\
\hline $\mathrm{CL} 2 \mathrm{~A}-\mathrm{NI} 2-\mathrm{P} 2$ & $95.26(6)$ \\
\hline $\mathrm{C} 23-\mathrm{NI} 2-\mathrm{CL} 2 \mathrm{~B}$ & $160.74(17)$ \\
\hline $\mathrm{C} 22-\mathrm{NI} 2-\mathrm{CL} 2 \mathrm{~B}$ & $120.92(16)$ \\
\hline $\mathrm{C} 21-\mathrm{NI} 2-\mathrm{CL} 2 \mathrm{~B}$ & $95.72(17)$ \\
\hline CL2A-NI2-CL2B & $15.97(10)$ \\
\hline $\mathrm{P} 2-\mathrm{NI} 2-\mathrm{CL} 2 \mathrm{~B}$ & $98.14(16)$ \\
\hline $\mathrm{C} 23-\mathrm{NI} 2-\mathrm{C} 23 \mathrm{~A}$ & $37.88(14)$ \\
\hline $\mathrm{C} 22-\mathrm{NI} 2-\mathrm{C} 23 \mathrm{~A}$ & $63.24(14)$ \\
\hline $\mathrm{C} 21-\mathrm{NI} 2-\mathrm{C} 23 \mathrm{~A}$ & $62.46(12)$ \\
\hline CL2A-NI2-C23A & $129.62(11)$ \\
\hline $\mathrm{P} 2-\mathrm{NI} 2-\mathrm{C} 23 \mathrm{~A}$ & $108.18(9)$ \\
\hline CL2B-NI2-C23A & $140.83(18)$ \\
\hline $\mathrm{C} 23-\mathrm{NI} 2-\mathrm{C} 27 \mathrm{~A}$ & $62.37(12)$ \\
\hline $\mathrm{C} 22-\mathrm{NI} 2-\mathrm{C} 27 \mathrm{~A}$ & $62.55(13)$ \\
\hline $\mathrm{C} 21-\mathrm{NI} 2-\mathrm{C} 27 \mathrm{~A}$ & $37.47(12)$ \\
\hline $\mathrm{CL} 2 \mathrm{~A}-\mathrm{NI} 2-\mathrm{C} 27 \mathrm{~A}$ & $101.80(9)$ \\
\hline $\mathrm{P} 2-\mathrm{NI} 2-\mathrm{C} 27 \mathrm{~A}$ & $139.11(9)$ \\
\hline $\mathrm{CL} 2 \mathrm{~B}-\mathrm{NI} 2-\mathrm{C} 27 \mathrm{~A}$ & $108.37(18)$ \\
\hline $\mathrm{C} 23 \mathrm{~A}-\mathrm{NI} 2-\mathrm{C} 27 \mathrm{~A}$ & $34.87(11)$ \\
\hline CL2B $-\mathrm{CL} 2 \mathrm{~A}-\mathrm{NI} 2$ & $83.69(19)$ \\
\hline CL2A-CL2B-NI2 & $80.3(2)$ \\
\hline C291-P2-C271 & $92.1(6)$ \\
\hline $\mathrm{C} 291-\mathrm{P} 2-\mathrm{C} 231$ & $84.9(7)$ \\
\hline $\mathrm{C} 271-\mathrm{P} 2-\mathrm{C} 231$ & $9.2(8)$ \\
\hline $\mathrm{C} 291-\mathrm{P} 2-\mathrm{C} 241$ & $102.6(5)$ \\
\hline $\mathrm{C} 271-\mathrm{P} 2-\mathrm{C} 241$ & $104.9(6)$ \\
\hline $\mathrm{C} 231-\mathrm{P} 2-\mathrm{C} 241$ & $112.0(5)$ \\
\hline $\mathrm{C} 291-\mathrm{P} 2-\mathrm{C} 281$ & $104.3(9)$ \\
\hline $\mathrm{C} 271-\mathrm{P} 2-\mathrm{C} 281$ & $13.1(6)$ \\
\hline $\mathrm{C} 231-\mathrm{P} 2-\mathrm{C} 281$ & $22.1(1)$ \\
\hline $\mathrm{C} 241-\mathrm{P} 2-\mathrm{C} 281$ & $97.1(8)$ \\
\hline $\mathrm{C} 291-\mathrm{P} 2-\mathrm{C} 251$ & $110.5(8)$ \\
\hline $\mathrm{C} 271-\mathrm{P} 2-\mathrm{C} 251$ & $103.8(6)$ \\
\hline $\mathrm{C} 231-\mathrm{P} 2-\mathrm{C} 251$ & $111.7(6)$ \\
\hline $\mathrm{C} 241-\mathrm{P} 2-\mathrm{C} 251$ & $7.9(8)$ \\
\hline $\mathrm{C} 281-\mathrm{P} 2-\mathrm{C} 251$ & $94.6(9)$ \\
\hline $\mathrm{C} 291-\mathrm{P} 2-\mathrm{C} 261$ & $103.4(7)$ \\
\hline $\mathrm{C} 271-\mathrm{P} 2-\mathrm{C} 261$ & $12.0(8)$ \\
\hline $\mathrm{C} 231-\mathrm{P} 2-\mathrm{C} 261$ & $18.6(7)$ \\
\hline $\mathrm{C} 241-\mathrm{P} 2-\mathrm{C} 261$ & $106.0(9)$ \\
\hline $\mathrm{C} 281-\mathrm{P} 2-\mathrm{C} 261$ & $9.10(14)$ \\
\hline $\mathrm{C} 251-\mathrm{P} 2-\mathrm{C} 261$ & $103.3(1)$ \\
\hline $\mathrm{C} 291-\mathrm{P} 2-\mathrm{C} 221$ & $12.4(5)$ \\
\hline $\mathrm{C} 271-\mathrm{P} 2-\mathrm{C} 221$ & $104.5(5)$ \\
\hline $\mathrm{C} 231-\mathrm{P} 2-\mathrm{C} 221$ & $97.2(6)$ \\
\hline $\mathrm{C} 241-\mathrm{P} 2-\mathrm{C} 221$ & $98.8(4)$ \\
\hline $\mathrm{C} 281-\mathrm{P} 2-\mathrm{C} 221$ & $116.6(7)$ \\
\hline $\mathrm{C} 251-\mathrm{P} 2-\mathrm{C} 221$ & $106.6(7)$ \\
\hline $\mathrm{C} 261-\mathrm{P} 2-\mathrm{C} 221$ & $115.8(6)$ \\
\hline C291—P2—NI2 & $119.8(6)$ \\
\hline $\mathrm{C} 271-\mathrm{P} 2-\mathrm{NI} 2$ & $118.3(5)$ \\
\hline $\mathrm{C} 231-\mathrm{P} 2-\mathrm{NI} 2$ & $117.6(6)$ \\
\hline $\mathrm{C} 241-\mathrm{P} 2-\mathrm{NI} 2$ & $115.53(19)$ \\
\hline
\end{tabular}




\begin{tabular}{|c|c|}
\hline $\mathrm{C} 281-\mathrm{P} 2-\mathrm{NI} 2$ & $114.5(7)$ \\
\hline $\mathrm{C} 251-\mathrm{P} 2-\mathrm{NI} 2$ & $110.2(4)$ \\
\hline $\mathrm{C} 261-\mathrm{P} 2-\mathrm{NI} 2$ & $108.1(7)$ \\
\hline $\mathrm{C} 221-\mathrm{P} 2-\mathrm{NI} 2$ & $112.4(3)$ \\
\hline $\mathrm{C} 213-\mathrm{SI} 2-\mathrm{C} 212$ & $109.5(3)$ \\
\hline $\mathrm{C} 213-\mathrm{SI} 2-\mathrm{C} 211$ & $110.1(2)$ \\
\hline $\mathrm{C} 212-\mathrm{SI} 2-\mathrm{C} 211$ & $109.7(2)$ \\
\hline $\mathrm{C} 213-\mathrm{SI} 2-\mathrm{C} 21$ & $108.63(19$ \\
\hline $\mathrm{C} 212-\mathrm{SI} 2-\mathrm{C} 21$ & $112.54(18$ \\
\hline $\mathrm{C} 211-\mathrm{SI} 2-\mathrm{C} 21$ & $106.40(17$ \\
\hline $\mathrm{C} 22-\mathrm{C} 21-\mathrm{C} 27 \mathrm{~A}$ & $107.1(3)$ \\
\hline $\mathrm{C} 22-\mathrm{C} 21-\mathrm{SI} 2$ & $124.9(3)$ \\
\hline $\mathrm{C} 27 \mathrm{~A}-\mathrm{C} 21-\mathrm{SI} 2$ & $126.8(2)$ \\
\hline $\mathrm{C} 22-\mathrm{C} 21-\mathrm{NI} 2$ & $67.46(15$ \\
\hline $\mathrm{C} 27 \mathrm{~A}-\mathrm{C} 21-\mathrm{NI} 2$ & $80.14(16$ \\
\hline $\mathrm{SI} 2-\mathrm{C} 21-\mathrm{NI} 2$ & $127.32(15$ \\
\hline $\mathrm{C} 23-\mathrm{C} 22-\mathrm{C} 21$ & $108.2(3)$ \\
\hline $\mathrm{C} 23-\mathrm{C} 22-\mathrm{NI} 2$ & $69.05(16$ \\
\hline $\mathrm{C} 21-\mathrm{C} 22-\mathrm{NI} 2$ & $73.16(16$ \\
\hline $\mathrm{C} 23-\mathrm{C} 22-\mathrm{H} 22$ & 125.9 \\
\hline $\mathrm{C} 21-\mathrm{C} 22-\mathrm{H} 22$ & 125.9 \\
\hline $\mathrm{N} 12-\mathrm{C} 22-\mathrm{H} 22$ & 123.5 \\
\hline $\mathrm{C} 22-\mathrm{C} 23-\mathrm{C} 23 \mathrm{~A}$ & $108.4(3)$ \\
\hline $\mathrm{C} 22-\mathrm{C} 23-\mathrm{NI} 2$ & $70.70(17$ \\
\hline $\mathrm{C} 23 \mathrm{~A}-\mathrm{C} 23-\mathrm{NI} 2$ & $82.40(18$ \\
\hline $\mathrm{C} 22-\mathrm{C} 23-\mathrm{H} 23$ & 125.8 \\
\hline $\mathrm{C} 23 \mathrm{~A}-\mathrm{C} 23-\mathrm{H} 23$ & 125.8 \\
\hline $\mathrm{NI} 2-\mathrm{C} 23-\mathrm{H} 23$ & 113.3 \\
\hline $\mathrm{C} 25-\mathrm{C} 24-\mathrm{C} 23 \mathrm{~A}$ & $118.5(5)$ \\
\hline $\mathrm{C} 25-\mathrm{C} 24-\mathrm{H} 24$ & 120.8 \\
\hline $\mathrm{C} 23 \mathrm{~A}-\mathrm{C} 24-\mathrm{H} 24$ & 120.8 \\
\hline $\mathrm{C} 26-\mathrm{C} 25-\mathrm{C} 24$ & $122.1(5)$ \\
\hline $\mathrm{C} 26-\mathrm{C} 25-\mathrm{H} 25$ & 119 \\
\hline $\mathrm{C} 24-\mathrm{C} 25-\mathrm{H} 25$ & 119 \\
\hline $\mathrm{C} 25-\mathrm{C} 26-\mathrm{C} 27$ & $122.2(6)$ \\
\hline $\mathrm{C} 25-\mathrm{C} 26-\mathrm{H} 26$ & 118.9 \\
\hline $\mathrm{C} 27-\mathrm{C} 26-\mathrm{H} 26$ & 118.9 \\
\hline $\mathrm{C} 26-\mathrm{C} 27-\mathrm{C} 27 \mathrm{~A}$ & $118.4(5)$ \\
\hline $\mathrm{C} 26-\mathrm{C} 27-\mathrm{H} 27$ & 120.8 \\
\hline $\mathrm{C} 27 \mathrm{~A}-\mathrm{C} 27-\mathrm{H} 27$ & 120.8 \\
\hline $\mathrm{SI} 2-\mathrm{C} 211-\mathrm{H} 21 \mathrm{~A}$ & 109.5 \\
\hline SI2-C211-H $21 B$ & 109.5 \\
\hline $\mathrm{H} 21 \mathrm{~A}-\mathrm{C} 211-\mathrm{H} 21 \mathrm{~B}$ & 109.5 \\
\hline SI2-C211-H21C & 109.5 \\
\hline $\mathrm{H} 21 \mathrm{~A}-\mathrm{C} 211-\mathrm{H} 21 \mathrm{C}$ & 109.5 \\
\hline $\mathrm{H} 21 \mathrm{~B}-\mathrm{C} 211-\mathrm{H} 21 \mathrm{C}$ & 109.5 \\
\hline SI2-C212-H21D & 109.5 \\
\hline $\mathrm{SI} 2-\mathrm{C} 212-\mathrm{H} 21 \mathrm{E}$ & 109.5 \\
\hline $\mathrm{H} 21 \mathrm{D}-\mathrm{C} 212-\mathrm{H} 21 \mathrm{E}$ & 109.5 \\
\hline $\mathrm{SI} 2-\mathrm{C} 212-\mathrm{H} 21 \mathrm{~F}$ & 109.5 \\
\hline $\mathrm{H} 21 \mathrm{D}-\mathrm{C} 212-\mathrm{H} 21 \mathrm{~F}$ & 109.5 \\
\hline $\mathrm{H} 21 \mathrm{E}-\mathrm{C} 212-\mathrm{H} 21 \mathrm{~F}$ & 109.5 \\
\hline $\mathrm{SI} 2-\mathrm{C} 213-\mathrm{H} 21 \mathrm{G}$ & 109.5 \\
\hline $\mathrm{SI} 2-\mathrm{C} 213-\mathrm{H} 21 \mathrm{H}$ & 109.5 \\
\hline $\mathrm{H} 21 \mathrm{G}-\mathrm{C} 213-\mathrm{H} 21 \mathrm{H}$ & 109.5 \\
\hline $\mathrm{SI} 2-\mathrm{C} 213-\mathrm{H} 21 \mathrm{I}$ & 109.5 \\
\hline $\mathrm{H} 21 \mathrm{G}-\mathrm{C} 213-\mathrm{H} 21 \mathrm{I}$ & 109.5 \\
\hline $\mathrm{H} 21 \mathrm{H}-\mathrm{C} 213-\mathrm{H} 21 \mathrm{I}$ & 109.5 \\
\hline $\mathrm{C} 236-\mathrm{C} 231-\mathrm{C} 232$ & $118.5(1)$ \\
\hline $\mathrm{C} 236-\mathrm{C} 231-\mathrm{P} 2$ & $119.9(9)$ \\
\hline $\mathrm{C} 232-\mathrm{C} 231-\mathrm{P} 2$ & $115.6(9)$ \\
\hline $\mathrm{C} 233-\mathrm{C} 232-\mathrm{C} 231$ & $120.0(1)$ \\
\hline
\end{tabular}

\begin{tabular}{|c|c|}
\hline $\mathrm{C} 233-\mathrm{C} 232-\mathrm{H} 232$ & 120 \\
\hline $\mathrm{C} 231-\mathrm{C} 232-\mathrm{H} 232$ & 120 \\
\hline $\mathrm{C} 232-\mathrm{C} 233-\mathrm{C} 234$ & $114.60(12)$ \\
\hline $\mathrm{C} 232-\mathrm{C} 233-\mathrm{H} 233$ & 122.7 \\
\hline $\mathrm{C} 234-\mathrm{C} 233-\mathrm{H} 233$ & 122.7 \\
\hline $\mathrm{C} 235-\mathrm{C} 234-\mathrm{C} 233$ & $123.30(14)$ \\
\hline $\mathrm{C} 235-\mathrm{C} 234-\mathrm{H} 234$ & 118.3 \\
\hline C233- $2234-\mathrm{H} 234$ & 118.3 \\
\hline $\mathrm{C} 234-\mathrm{C} 235-\mathrm{C} 236$ & $118.10(12)$ \\
\hline $\mathrm{C} 234-\mathrm{C} 235-\mathrm{H} 235$ & 121 \\
\hline $\mathrm{C} 236-\mathrm{C} 235-\mathrm{H} 235$ & 121 \\
\hline $\mathrm{C} 231-\mathrm{C} 236-\mathrm{C} 235$ & $115.8(1)$ \\
\hline $\mathrm{C} 231-\mathrm{C} 236-\mathrm{H} 236$ & 122.1 \\
\hline $\mathrm{C} 235-\mathrm{C} 236-\mathrm{H} 236$ & 122.1 \\
\hline $\mathrm{C} 262-\mathrm{C} 261-\mathrm{C} 266$ & $111.40(11)$ \\
\hline $\mathrm{C} 262-\mathrm{C} 261-\mathrm{P} 2$ & $127.70(11)$ \\
\hline $\mathrm{C} 266-\mathrm{C} 261-\mathrm{P} 2$ & $121.00(12)$ \\
\hline $\mathrm{C} 263-\mathrm{C} 262-\mathrm{C} 261$ & $126.60(13)$ \\
\hline $\mathrm{C} 263-\mathrm{C} 262-\mathrm{H} 262$ & 116.7 \\
\hline $\mathrm{C} 261-\mathrm{C} 262-\mathrm{H} 262$ & 116.7 \\
\hline $\mathrm{C} 264-\mathrm{C} 263-\mathrm{C} 262$ & $119.30(15)$ \\
\hline $\mathrm{C} 264-\mathrm{C} 263-\mathrm{H} 263$ & 120.3 \\
\hline $\mathrm{C} 262-\mathrm{C} 263-\mathrm{H} 263$ & 120.3 \\
\hline $\mathrm{C} 263-\mathrm{C} 264-\mathrm{C} 265$ & $119.30(15)$ \\
\hline $\mathrm{C} 263-\mathrm{C} 264-\mathrm{H} 264$ & 120.3 \\
\hline $\mathrm{C} 265-\mathrm{C} 264-\mathrm{H} 264$ & 120.3 \\
\hline $\mathrm{C} 264-\mathrm{C} 265-\mathrm{C} 266$ & $118.80(16)$ \\
\hline $\mathrm{C} 264-\mathrm{C} 265-\mathrm{H} 265$ & 120.6 \\
\hline C266- C265-H265 & 120.6 \\
\hline $\mathrm{C} 261-\mathrm{C} 266-\mathrm{C} 265$ & $123.80(15)$ \\
\hline $\mathrm{C} 261-\mathrm{C} 266-\mathrm{H} 266$ & 118.1 \\
\hline $\mathrm{C} 265-\mathrm{C} 266-\mathrm{H} 266$ & 118.1 \\
\hline $\mathrm{C} 272-\mathrm{C} 271-\mathrm{C} 276$ & $105.5(9)$ \\
\hline $\mathrm{C} 272-\mathrm{C} 271-\mathrm{P} 2$ & $126.80(11)$ \\
\hline $\mathrm{C} 276-\mathrm{C} 271-\mathrm{P} 2$ & $112.4(1)$ \\
\hline $\mathrm{C} 271-\mathrm{C} 272-\mathrm{C} 273$ & $128.30(14)$ \\
\hline $\mathrm{C} 271-\mathrm{C} 272-\mathrm{H} 272$ & 115.8 \\
\hline $\mathrm{C} 273-\mathrm{C} 272-\mathrm{H} 272$ & 115.8 \\
\hline $\mathrm{C} 274-\mathrm{C} 273-\mathrm{C} 272$ & $117.10(15)$ \\
\hline $\mathrm{C} 274-\mathrm{C} 273-\mathrm{H} 273$ & 121.5 \\
\hline $\mathrm{C} 272-\mathrm{C} 273-\mathrm{H} 273$ & 121.5 \\
\hline $\mathrm{C} 273-\mathrm{C} 274-\mathrm{C} 275$ & $110.40(14)$ \\
\hline $\mathrm{C} 273-\mathrm{C} 274-\mathrm{H} 274$ & 124.8 \\
\hline $\mathrm{C} 275-\mathrm{C} 274-\mathrm{H} 274$ & 124.8 \\
\hline $\mathrm{C} 274-\mathrm{C} 275-\mathrm{C} 276$ & $126.10(12)$ \\
\hline $\mathrm{C} 274-\mathrm{C} 275-\mathrm{H} 275$ & 117 \\
\hline $\mathrm{C} 276-\mathrm{C} 275-\mathrm{H} 275$ & 117 \\
\hline $\mathrm{C} 271-\mathrm{C} 276-\mathrm{C} 275$ & $115.8(1)$ \\
\hline $\mathrm{C} 271-\mathrm{C} 276-\mathrm{H} 276$ & 122.1 \\
\hline $\mathrm{C} 275-\mathrm{C} 276-\mathrm{H} 276$ & 122.1 \\
\hline $\mathrm{C} 282-\mathrm{C} 281-\mathrm{C} 286$ & $103.20(15)$ \\
\hline $\mathrm{C} 282-\mathrm{C} 281-\mathrm{P} 2$ & $133.10(14)$ \\
\hline $\mathrm{C} 286-\mathrm{C} 281-\mathrm{P} 2$ & $111.90(14)$ \\
\hline $\mathrm{C} 281-\mathrm{C} 282-\mathrm{C} 283$ & $124.10(14)$ \\
\hline $\mathrm{C} 281-\mathrm{C} 282-\mathrm{H} 282$ & 117.9 \\
\hline $\mathrm{C} 283-\mathrm{C} 282-\mathrm{H} 282$ & 117.9 \\
\hline $\mathrm{C} 284-\mathrm{C} 283-\mathrm{C} 282$ & $118.60(16)$ \\
\hline $\mathrm{C} 284-\mathrm{C} 283-\mathrm{H} 283$ & 120.7 \\
\hline $\mathrm{C} 282-\mathrm{C} 283-\mathrm{H} 283$ & 120.7 \\
\hline $\mathrm{C} 285-\mathrm{C} 284-\mathrm{C} 283$ & $111.60(15)$ \\
\hline $\mathrm{C} 285-\mathrm{C} 284-\mathrm{H} 284$ & 124.2 \\
\hline $\mathrm{C} 283-\mathrm{C} 284-\mathrm{H} 284$ & 124.2 \\
\hline
\end{tabular}




\begin{tabular}{|c|c|}
\hline $\mathrm{C} 284-\mathrm{C} 285-\mathrm{C} 286$ & $123.50(18)$ \\
\hline $\mathrm{C} 284-\mathrm{C} 285-\mathrm{H} 285$ & 118.2 \\
\hline $\mathrm{C} 286-\mathrm{C} 285-\mathrm{H} 285$ & 118.2 \\
\hline $\mathrm{C} 281-\mathrm{C} 286-\mathrm{C} 285$ & $112.70(14)$ \\
\hline $\mathrm{C} 281-\mathrm{C} 286-\mathrm{H} 286$ & 123.7 \\
\hline $\mathrm{C} 285-\mathrm{C} 286-\mathrm{H} 286$ & 123.7 \\
\hline C246-C241-C242 & $118.4(4)$ \\
\hline $\mathrm{C} 246-\mathrm{C} 241-\mathrm{P} 2$ & $125.5(5)$ \\
\hline $\mathrm{C} 242-\mathrm{C} 241-\mathrm{P} 2$ & $116.1(4)$ \\
\hline $\mathrm{C} 241-\mathrm{C} 242-\mathrm{C} 243$ & $120.2(4)$ \\
\hline $\mathrm{C} 241-\mathrm{C} 242-\mathrm{H} 242$ & 119.9 \\
\hline $\mathrm{C} 243-\mathrm{C} 242-\mathrm{H} 242$ & 119.9 \\
\hline $\mathrm{C} 244-\mathrm{C} 243-\mathrm{C} 242$ & $120.8(6)$ \\
\hline $\mathrm{C} 244-\mathrm{C} 243-\mathrm{H} 243$ & 119.6 \\
\hline $\mathrm{C} 242-\mathrm{C} 243-\mathrm{H} 243$ & 119.6 \\
\hline $\mathrm{C} 245-\mathrm{C} 244-\mathrm{C} 243$ & $118.9(8)$ \\
\hline $\mathrm{C} 245-\mathrm{C} 244-\mathrm{H} 244$ & 120.5 \\
\hline $\mathrm{C} 243-\mathrm{C} 244-\mathrm{H} 244$ & 120.5 \\
\hline $\mathrm{C} 244-\mathrm{C} 245-\mathrm{C} 246$ & $120.8(6)$ \\
\hline $\mathrm{C} 244-\mathrm{C} 245-\mathrm{H} 245$ & 119.6 \\
\hline C246-C245-H245 & 119.6 \\
\hline $\mathrm{C} 241-\mathrm{C} 246-\mathrm{C} 245$ & $120.8(5)$ \\
\hline $\mathrm{C} 241-\mathrm{C} 246-\mathrm{H} 246$ & 119.6 \\
\hline $\mathrm{C} 245-\mathrm{C} 246-\mathrm{H} 246$ & 119.6 \\
\hline C256-C251-C252 & $118.1(7)$ \\
\hline $\mathrm{C} 256-\mathrm{C} 251-\mathrm{P} 2$ & $117.1(6)$ \\
\hline $\mathrm{C} 252-\mathrm{C} 251-\mathrm{P} 2$ & $123.9(6)$ \\
\hline $\mathrm{C} 251-\mathrm{C} 252-\mathrm{C} 253$ & $121.1(8)$ \\
\hline $\mathrm{C} 251-\mathrm{C} 252-\mathrm{H} 252$ & 119.4 \\
\hline $\mathrm{C} 253-\mathrm{C} 252-\mathrm{H} 252$ & 119.4 \\
\hline $\mathrm{C} 254-\mathrm{C} 253-\mathrm{C} 252$ & $119.5(1)$ \\
\hline $\mathrm{C} 254-\mathrm{C} 253-\mathrm{H} 253$ & 120.2 \\
\hline $\mathrm{C} 252-\mathrm{C} 253-\mathrm{H} 253$ & 120.2 \\
\hline $\mathrm{C} 255-\mathrm{C} 254-\mathrm{C} 253$ & $120.10(12)$ \\
\hline $\mathrm{C} 255-\mathrm{C} 254-\mathrm{H} 254$ & 120 \\
\hline C253-C254-H254 & 120 \\
\hline $\mathrm{C} 254-\mathrm{C} 255-\mathrm{C} 256$ & $120.30(11)$ \\
\hline $\mathrm{C} 254-\mathrm{C} 255-\mathrm{H} 255$ & 119.8 \\
\hline $\mathrm{C} 256-\mathrm{C} 255-\mathrm{H} 255$ & 119.8 \\
\hline $\mathrm{C} 251-\mathrm{C} 256-\mathrm{C} 255$ & $120.8(8)$ \\
\hline $\mathrm{C} 251-\mathrm{C} 256-\mathrm{H} 256$ & 119.6 \\
\hline $\mathrm{C} 255-\mathrm{C} 256-\mathrm{H} 256$ & 119.6 \\
\hline $\mathrm{C} 226-\mathrm{C} 221-\mathrm{C} 222$ & $112.1(6)$ \\
\hline $\mathrm{C} 226-\mathrm{C} 221-\mathrm{P} 2$ & $124.6(4)$ \\
\hline $\mathrm{C} 222-\mathrm{C} 221-\mathrm{P} 2$ & $123.2(5)$ \\
\hline $\mathrm{C} 223-\mathrm{C} 222-\mathrm{C} 221$ & $122.7(7)$ \\
\hline $\mathrm{C} 223-\mathrm{C} 222-\mathrm{H} 222$ & 118.6 \\
\hline $\mathrm{C} 221-\mathrm{C} 222-\mathrm{H} 222$ & 118.6 \\
\hline $\mathrm{C} 224-\mathrm{C} 223-\mathrm{C} 222$ & $121.8(9)$ \\
\hline $\mathrm{C} 224-\mathrm{C} 223-\mathrm{H} 223$ & 119.1 \\
\hline $\mathrm{C} 222-\mathrm{C} 223-\mathrm{H} 223$ & 119.1 \\
\hline $\mathrm{C} 13-\mathrm{NI} 1-\mathrm{P} 1-\mathrm{C} 151$ & $-119.6(6)$ \\
\hline $\mathrm{C} 12-\mathrm{NI} 1-\mathrm{P} 1-\mathrm{C} 151$ & $-91.8(6)$ \\
\hline $\mathrm{C} 11-\mathrm{NI} 1-\mathrm{P} 1-\mathrm{C} 151$ & $-136.8(6)$ \\
\hline CL1-NI1-P1-C151 & $59.2(6)$ \\
\hline $\mathrm{C} 13 \mathrm{~A}-\mathrm{NI} 1-\mathrm{P} 1-\mathrm{C} 151$ & $-158.4(6)$ \\
\hline $\mathrm{C} 17 \mathrm{~A}-\mathrm{NI} 1-\mathrm{P} 1-\mathrm{C} 151$ & $178.0(6)$ \\
\hline C13-NI1-P1-C131 & $131.3(5)$ \\
\hline $\mathrm{C} 12-\mathrm{NI} 1-\mathrm{P} 1-\mathrm{C} 131$ & $159.1(5)$ \\
\hline $\mathrm{C} 11-\mathrm{NI} 1-\mathrm{P} 1-\mathrm{C} 131$ & $114.1(5)$ \\
\hline CL1-NI1-P1-C131 & $-49.9(4)$ \\
\hline $\mathrm{C} 13 \mathrm{~A}-\mathrm{NI} 1-\mathrm{P} 1-\mathrm{C} 131$ & $92.5(5)$ \\
\hline
\end{tabular}

\begin{tabular}{|c|c|}
\hline $\mathrm{C} 225-\mathrm{C} 224-\mathrm{C} 223$ & $118.3(9)$ \\
\hline $\mathrm{C} 225-\mathrm{C} 224-\mathrm{H} 224$ & 120.9 \\
\hline $\mathrm{C} 223-\mathrm{C} 224-\mathrm{H} 224$ & 120.9 \\
\hline $\mathrm{C} 224-\mathrm{C} 225-\mathrm{C} 226$ & $118.2(8)$ \\
\hline $\mathrm{C} 224-\mathrm{C} 225-\mathrm{H} 225$ & 120.9 \\
\hline $\mathrm{C} 226-\mathrm{C} 225-\mathrm{H} 225$ & 120.9 \\
\hline $\mathrm{C} 221-\mathrm{C} 226-\mathrm{C} 225$ & $126.6(7)$ \\
\hline $\mathrm{C} 221-\mathrm{C} 226-\mathrm{H} 226$ & 116.7 \\
\hline $\mathrm{C} 225-\mathrm{C} 226-\mathrm{H} 226$ & 116.7 \\
\hline C296-C291-C292 & $129.6(1)$ \\
\hline $\mathrm{C} 296-\mathrm{C} 291-\mathrm{P} 2$ & $113.5(7)$ \\
\hline $\mathrm{C} 292-\mathrm{C} 291-\mathrm{P} 2$ & $112.9(8)$ \\
\hline C291-C292-C293 & $113.2(9)$ \\
\hline C291-C292-H292 & 123.4 \\
\hline C293-C292-H292 & 123.4 \\
\hline C294-C293-C292 & $120.70(11)$ \\
\hline $\mathrm{C} 294-\mathrm{C} 293-\mathrm{H} 293$ & 119.6 \\
\hline C292-C293-H293 & 119.6 \\
\hline $\mathrm{C} 295-\mathrm{C} 294-\mathrm{C} 293$ & $120.30(11)$ \\
\hline C295-C294-H294 & 119.9 \\
\hline C293-C294-H294 & 119.9 \\
\hline C294-C295-C296 & $123.60(11)$ \\
\hline $\mathrm{C} 294-\mathrm{C} 295-\mathrm{H} 295$ & 118.2 \\
\hline $\mathrm{C} 296-\mathrm{C} 295-\mathrm{H} 295$ & 118.2 \\
\hline $\mathrm{C} 291-\mathrm{C} 296-\mathrm{C} 295$ & $110.9(1)$ \\
\hline $\mathrm{C} 291-\mathrm{C} 296-\mathrm{H} 296$ & 124.6 \\
\hline $\mathrm{C} 295-\mathrm{C} 296-\mathrm{H} 296$ & 124.6 \\
\hline $\mathrm{C} 14-\mathrm{C} 13 \mathrm{~A}-\mathrm{C} 17 \mathrm{~A}$ & $120.7(3)$ \\
\hline $\mathrm{C} 14-\mathrm{C} 13 \mathrm{~A}-\mathrm{C} 13$ & $132.3(3)$ \\
\hline $\mathrm{C} 17 \mathrm{~A}-\mathrm{C} 13 \mathrm{~A}-\mathrm{C} 13$ & $107.0(2)$ \\
\hline $\mathrm{C} 14-\mathrm{C} 13 \mathrm{~A}-\mathrm{NI} 1$ & $130.35(19)$ \\
\hline $\mathrm{C} 17 \mathrm{~A}-\mathrm{C} 13 \mathrm{~A}-\mathrm{NI} 1$ & $72.75(14)$ \\
\hline C13-C13A-NI1 & $60.79(14)$ \\
\hline $\mathrm{C} 17-\mathrm{C} 17 \mathrm{~A}-\mathrm{C} 13 \mathrm{~A}$ & $119.7(3)$ \\
\hline $\mathrm{C} 17-\mathrm{C} 17 \mathrm{~A}-\mathrm{C} 11$ & $131.8(2)$ \\
\hline $\mathrm{C} 13 \mathrm{~A}-\mathrm{C} 17 \mathrm{~A}-\mathrm{C} 11$ & $108.4(2)$ \\
\hline C17-C17A-NI1 & $129.17(18)$ \\
\hline $\mathrm{C} 13 \mathrm{~A}-\mathrm{C} 17 \mathrm{~A}-\mathrm{NI} 1$ & $71.84(15)$ \\
\hline C11-C17A-NI1 & $63.28(12)$ \\
\hline $\mathrm{C} 27 \mathrm{~A}-\mathrm{C} 23 \mathrm{~A}-\mathrm{C} 24$ & $118.8(4)$ \\
\hline $\mathrm{C} 27 \mathrm{~A}-\mathrm{C} 23 \mathrm{~A}-\mathrm{C} 23$ & $107.1(3)$ \\
\hline $\mathrm{C} 24-\mathrm{C} 23 \mathrm{~A}-\mathrm{C} 23$ & $134.0(4)$ \\
\hline $\mathrm{C} 27 \mathrm{~A}-\mathrm{C} 23 \mathrm{~A}-\mathrm{NI} 2$ & $73.89(17)$ \\
\hline $\mathrm{C} 24-\mathrm{C} 23 \mathrm{~A}-\mathrm{NI} 2$ & $130.7(2)$ \\
\hline $\mathrm{C} 23-\mathrm{C} 23 \mathrm{~A}-\mathrm{NI} 2$ & $59.72(16)$ \\
\hline $\mathrm{C} 27-\mathrm{C} 27 \mathrm{~A}-\mathrm{C} 23 \mathrm{~A}$ & $119.9(3)$ \\
\hline $\mathrm{C} 27-\mathrm{C} 27 \mathrm{~A}-\mathrm{C} 21$ & $132.2(3)$ \\
\hline $\mathrm{C} 23 \mathrm{~A}-\mathrm{C} 27 \mathrm{~A}-\mathrm{C} 21$ & $107.9(3)$ \\
\hline $\mathrm{C} 27-\mathrm{C} 27 \mathrm{~A}-\mathrm{NI} 2$ & $130.5(2)$ \\
\hline $\mathrm{C} 23 \mathrm{~A}-\mathrm{C} 27 \mathrm{~A}-\mathrm{NI} 2$ & $71.25(16)$ \\
\hline $\mathrm{C} 21-\mathrm{C} 27 \mathrm{~A}-\mathrm{NI} 2$ & $62.39(14)$ \\
\hline $\mathrm{C} 17 \mathrm{~A}-\mathrm{NI} 1-\mathrm{P} 1-\mathrm{C} 131$ & $68.9(5)$ \\
\hline $\mathrm{C} 13-\mathrm{NI} 1-\mathrm{P} 1-\mathrm{C} 121$ & $10.45(14)$ \\
\hline $\mathrm{C} 12-\mathrm{NI} 1-\mathrm{P} 1-\mathrm{C} 121$ & $38.23(15)$ \\
\hline $\mathrm{C} 11-\mathrm{NI} 1-\mathrm{P} 1-\mathrm{C} 121$ & $-6.8(3)$ \\
\hline CL1-NI1-P1-C121 & $-170.82(9)$ \\
\hline $\mathrm{C} 13 \mathrm{~A}-\mathrm{NI} 1-\mathrm{P} 1-\mathrm{C} 121$ & $-28.39(13)$ \\
\hline $\mathrm{C} 17 \mathrm{~A}-\mathrm{NI} 1-\mathrm{P} 1-\mathrm{C} 121$ & $-52.03(13)$ \\
\hline C13-NI1-P1-C141 & $-106.6(2)$ \\
\hline $\mathrm{C} 12-\mathrm{NI} 1-\mathrm{P} 1-\mathrm{C} 141$ & $-78.8(2)$ \\
\hline $\mathrm{C} 11-\mathrm{NI} 1-\mathrm{P} 1-\mathrm{C} 141$ & $-123.8(4)$ \\
\hline $\mathrm{CL} 1-\mathrm{NI} 1-\mathrm{P} 1-\mathrm{C} 141$ & $72.2(2)$ \\
\hline
\end{tabular}




\begin{tabular}{|c|c|}
\hline $\mathrm{C} 13 \mathrm{~A}-\mathrm{NI} 1-\mathrm{P} 1-\mathrm{C} 141$ & $-145.4(2)$ \\
\hline $\mathrm{C} 17 \mathrm{~A}-\mathrm{NI} 1-\mathrm{P} 1-\mathrm{C} 141$ & $-169.0(2)$ \\
\hline $\mathrm{C} 13-\mathrm{NI} 1-\mathrm{P} 1-\mathrm{C} 161$ & $122.40(12)$ \\
\hline $\mathrm{C} 12-\mathrm{NI} 1-\mathrm{P} 1-\mathrm{C} 161$ & $150.20(12)$ \\
\hline $\mathrm{C} 11-\mathrm{NI} 1-\mathrm{P} 1-\mathrm{C} 161$ & $105.10(13)$ \\
\hline CL1-NI1-P1-C161 & $-58.90(12)$ \\
\hline $\mathrm{C} 13 \mathrm{~A}-\mathrm{NI} 1-\mathrm{P} 1-\mathrm{C} 161$ & $83.60(12)$ \\
\hline $\mathrm{C} 17 \mathrm{~A}-\mathrm{NI} 1-\mathrm{P} 1-\mathrm{C} 161$ & $59.90(12)$ \\
\hline $\mathrm{C} 112-\mathrm{SI} 1-\mathrm{C} 11-\mathrm{C} 12$ & $159.4(2)$ \\
\hline $\mathrm{C} 113-\mathrm{SI} 1-\mathrm{C} 11-\mathrm{C} 12$ & $36.3(2)$ \\
\hline C111-SI1-C11-C12 & $-81.4(2)$ \\
\hline $\mathrm{C} 112-\mathrm{SI} 1-\mathrm{C} 11-\mathrm{C} 17 \mathrm{~A}$ & $-34.2(3)$ \\
\hline $\mathrm{C} 113-\mathrm{SI} 1-\mathrm{C} 11-\mathrm{C} 17 \mathrm{~A}$ & $-157.2(2)$ \\
\hline $\mathrm{C} 111-\mathrm{SI} 1-\mathrm{C} 11-\mathrm{C} 17 \mathrm{~A}$ & $85.0(2)$ \\
\hline C112-SI1-C11—NI1 & $72.9(2)$ \\
\hline C113-SI1-C11-NI1 & $-50.1(2)$ \\
\hline C111-SI1-C11-NI1 & $-167.82(15$ \\
\hline $\mathrm{C} 13-\mathrm{NI} 1-\mathrm{C} 11-\mathrm{C} 12$ & $37.31(18$ \\
\hline CL1-NI1-C11-C12 & $-140.17(15$ \\
\hline $\mathrm{P} 1-\mathrm{NI} 1-\mathrm{C} 11-\mathrm{C} 12$ & $55.8(4)$ \\
\hline $\mathrm{C} 13 \mathrm{~A}-\mathrm{NI} 1-\mathrm{C} 11-\mathrm{C} 12$ & $79.32(18$ \\
\hline $\mathrm{C} 17 \mathrm{~A}-\mathrm{NI} 1-\mathrm{C} 11-\mathrm{C} 12$ & $112.5(2)$ \\
\hline $\mathrm{C} 13-\mathrm{NI} 1-\mathrm{C} 11-\mathrm{C} 17 \mathrm{~A}$ & $-75.16(17)$ \\
\hline $\mathrm{C} 12-\mathrm{NI} 1-\mathrm{C} 11-\mathrm{C} 17 \mathrm{~A}$ & $-112.5(2)$ \\
\hline CL1-NI1-C11-C17A & $107.37(13)$ \\
\hline $\mathrm{P} 1-\mathrm{NI} 1-\mathrm{C} 11-\mathrm{C} 17 \mathrm{~A}$ & $-56.6(4)$ \\
\hline $\mathrm{C} 13 \mathrm{~A}-\mathrm{NI} 1-\mathrm{C} 11-\mathrm{C} 17 \mathrm{~A}$ & $-33.15(15)$ \\
\hline C13-NI1-C11-SI1 & $153.8(2)$ \\
\hline $\mathrm{C} 12-\mathrm{NI} 1-\mathrm{C} 11-\mathrm{SI} 1$ & $116.5(2)$ \\
\hline CL1-NI1-C11-SI1 & $-23.70(15)$ \\
\hline P1-NI1-C11-SI1 & $172.28(18)$ \\
\hline $\mathrm{C} 13 \mathrm{~A}-\mathrm{NI} 1-\mathrm{C} 11-\mathrm{SI} 1$ & $-164.2(2)$ \\
\hline C17A-NI1-C11-SI1 & $-131.1(2)$ \\
\hline $\mathrm{C} 17 \mathrm{~A}-\mathrm{C} 11-\mathrm{C} 12-\mathrm{C} 13$ & $10.5(3)$ \\
\hline $\mathrm{SI} 1-\mathrm{C} 11-\mathrm{C} 12-\mathrm{C} 13$ & $179.59(17)$ \\
\hline $\mathrm{NI} 1-\mathrm{C} 11-\mathrm{C} 12-\mathrm{C} 13$ & $-59.87(18)$ \\
\hline $\mathrm{C} 17 \mathrm{~A}-\mathrm{C} 11-\mathrm{C} 12-\mathrm{NI} 1$ & $70.34(15)$ \\
\hline SI1-C11-C12-NI1 & $-120.54(17)$ \\
\hline $\mathrm{C} 11-\mathrm{NI} 1-\mathrm{C} 12-\mathrm{C} 13$ & $119.4(2)$ \\
\hline $\mathrm{CL} 1-\mathrm{NI} 1-\mathrm{C} 12-\mathrm{C} 13$ & $170.18(15)$ \\
\hline $\mathrm{P} 1-\mathrm{NI} 1-\mathrm{C} 12-\mathrm{C} 13$ & $-45.8(2)$ \\
\hline $\mathrm{C} 13 \mathrm{~A}-\mathrm{NI} 1-\mathrm{C} 12-\mathrm{C} 13$ & $39.60(17)$ \\
\hline $\mathrm{C} 17 \mathrm{~A}-\mathrm{NI} 1-\mathrm{C} 12-\mathrm{C} 13$ & $79.50(19)$ \\
\hline $\mathrm{C} 13-\mathrm{NI} 1-\mathrm{C} 12-\mathrm{C} 11$ & $-119.4(2)$ \\
\hline $\mathrm{CL} 1-\mathrm{NI} 1-\mathrm{C} 12-\mathrm{C} 11$ & $50.82(18)$ \\
\hline $\mathrm{P} 1-\mathrm{NI} 1-\mathrm{C} 12-\mathrm{C} 11$ & $-165.14(11$ \\
\hline $\mathrm{C} 13 \mathrm{~A}-\mathrm{NI} 1-\mathrm{C} 12-\mathrm{C} 11$ & $-79.76(17$ \\
\hline $\mathrm{C} 17 \mathrm{~A}-\mathrm{NI} 1-\mathrm{C} 12-\mathrm{C} 11$ & $-39.86(14)$ \\
\hline $\mathrm{C} 11-\mathrm{C} 12-\mathrm{C} 13-\mathrm{C} 13 \mathrm{~A}$ & $-11.7(3)$ \\
\hline $\mathrm{NI} 1-\mathrm{C} 12-\mathrm{C} 13-\mathrm{C} 13 \mathrm{~A}$ & $-73.36(18)$ \\
\hline $\mathrm{C} 11-\mathrm{C} 12-\mathrm{C} 13-\mathrm{NI} 1$ & $61.70(18)$ \\
\hline $\mathrm{C} 11-\mathrm{NI} 1-\mathrm{C} 13-\mathrm{C} 12$ & $-37.15(16)$ \\
\hline CL1-NI1-C13-C12 & $-28.6(5)$ \\
\hline $\mathrm{P} 1-\mathrm{NI} 1-\mathrm{C} 13-\mathrm{C} 12$ & $147.13(16)$ \\
\hline $\mathrm{C} 13 \mathrm{~A}-\mathrm{NI} 1-\mathrm{C} 13-\mathrm{C} 12$ & $-112.8(2)$ \\
\hline $\mathrm{C} 17 \mathrm{~A}-\mathrm{NI} 1-\mathrm{C} 13-\mathrm{C} 12$ & $-79.20(17)$ \\
\hline $\mathrm{C} 12-\mathrm{NI} 1-\mathrm{C} 13-\mathrm{C} 13 \mathrm{~A}$ & $112.8(2)$ \\
\hline $\mathrm{C} 11-\mathrm{NI} 1-\mathrm{C} 13-\mathrm{C} 13 \mathrm{~A}$ & $75.68(16$ \\
\hline CL1-NI1-C13-C13A & $84.3(4)$ \\
\hline $\mathrm{P} 1-\mathrm{NI} 1-\mathrm{C} 13-\mathrm{C} 13 \mathrm{~A}$ & $-100.04(14)$ \\
\hline $\mathrm{C} 17 \mathrm{~A}-\mathrm{NI} 1-\mathrm{C} 13-\mathrm{C} 13 \mathrm{~A}$ & $33.64(14)$ \\
\hline $\mathrm{C} 13 \mathrm{~A}-\mathrm{C} 14-\mathrm{C} 15-\mathrm{C} 16$ & $2.6(5)$ \\
\hline
\end{tabular}

\begin{tabular}{|c|c|}
\hline $\mathrm{C} 14-\mathrm{C} 15-\mathrm{C} 16-\mathrm{C} 17$ & $-1.6(6)$ \\
\hline $\mathrm{C} 15-\mathrm{C} 16-\mathrm{C} 17-\mathrm{C} 17 \mathrm{~A}$ & $-1.2(5)$ \\
\hline $\mathrm{C} 151-\mathrm{P} 1-\mathrm{C} 121-\mathrm{C} 122$ & $-130.6(4)$ \\
\hline $\mathrm{C} 131-\mathrm{P} 1-\mathrm{C} 121-\mathrm{C} 122$ & $-27.3(4)$ \\
\hline $\mathrm{C} 141-\mathrm{P} 1-\mathrm{C} 121-\mathrm{C} 122$ & $-136.6(3)$ \\
\hline $\mathrm{C} 161-\mathrm{P} 1-\mathrm{C} 121-\mathrm{C} 122$ & $-25.0(9)$ \\
\hline $\mathrm{NI} 1-\mathrm{P} 1-\mathrm{C} 121-\mathrm{C} 122$ & $98.8(2)$ \\
\hline $\mathrm{C} 151-\mathrm{P} 1-\mathrm{C} 121-\mathrm{C} 126$ & $56.1(4)$ \\
\hline $\mathrm{C} 131-\mathrm{P} 1-\mathrm{C} 121-\mathrm{C} 126$ & $159.3(3)$ \\
\hline $\mathrm{C} 141-\mathrm{P} 1-\mathrm{C} 121-\mathrm{C} 126$ & $50.0(3)$ \\
\hline $\mathrm{C} 161-\mathrm{P} 1-\mathrm{C} 121-\mathrm{C} 126$ & $161.7(9)$ \\
\hline $\mathrm{NI} 1-\mathrm{P} 1-\mathrm{C} 121-\mathrm{C} 126$ & $-74.5(2)$ \\
\hline $\mathrm{C} 126-\mathrm{C} 121-\mathrm{C} 122-\mathrm{C} 123$ & $-0.5(5)$ \\
\hline $\mathrm{P} 1-\mathrm{C} 121-\mathrm{C} 122-\mathrm{C} 123$ & $-174.0(3)$ \\
\hline $\mathrm{C} 121-\mathrm{C} 122-\mathrm{C} 123-\mathrm{C} 124$ & $-0.9(6)$ \\
\hline $\mathrm{C} 122-\mathrm{C} 123-\mathrm{C} 124-\mathrm{C} 125$ & $1.8(6)$ \\
\hline $\mathrm{C} 123-\mathrm{C} 124-\mathrm{C} 125-\mathrm{C} 126$ & $-1.4(6)$ \\
\hline $\mathrm{C} 124-\mathrm{C} 125-\mathrm{C} 126-\mathrm{C} 121$ & $0.0(5)$ \\
\hline $\mathrm{C} 122-\mathrm{C} 121-\mathrm{C} 126-\mathrm{C} 125$ & $0.9(4)$ \\
\hline $\mathrm{P} 1-\mathrm{C} 121-\mathrm{C} 126-\mathrm{C} 125$ & $174.5(3)$ \\
\hline $\mathrm{C} 151-\mathrm{P} 1-\mathrm{C} 131-\mathrm{C} 132$ & $-137.3(1)$ \\
\hline $\mathrm{C} 121-\mathrm{P} 1-\mathrm{C} 131-\mathrm{C} 132$ & $108.1(8)$ \\
\hline $\mathrm{C} 141-\mathrm{P} 1-\mathrm{C} 131-\mathrm{C} 132$ & $-145.6(8)$ \\
\hline $\mathrm{C} 161-\mathrm{P} 1-\mathrm{C} 131-\mathrm{C} 132$ & $92(6)$ \\
\hline NI1-P1-C131-C132 & $-18.3(1)$ \\
\hline $\mathrm{C} 151-\mathrm{P} 1-\mathrm{C} 131-\mathrm{C} 136$ & $46.50(11)$ \\
\hline $\mathrm{C} 121-\mathrm{P} 1-\mathrm{C} 131-\mathrm{C} 136$ & $-68.0(1)$ \\
\hline $\mathrm{C} 141-\mathrm{P} 1-\mathrm{C} 131-\mathrm{C} 136$ & $38.3(1)$ \\
\hline $\mathrm{C} 161-\mathrm{P} 1-\mathrm{C} 131-\mathrm{C} 136$ & $-84(5)$ \\
\hline $\mathrm{NI} 1-\mathrm{P} 1-\mathrm{C} 131-\mathrm{C} 136$ & $165.5(7)$ \\
\hline $\mathrm{C} 136-\mathrm{C} 131-\mathrm{C} 132-\mathrm{C} 133$ & $3.90(15)$ \\
\hline $\mathrm{P} 1-\mathrm{C} 131-\mathrm{C} 132-\mathrm{C} 133$ & $-172.2(7)$ \\
\hline $\mathrm{C} 131-\mathrm{C} 132-\mathrm{C} 133-\mathrm{C} 134$ & $-3.40(13)$ \\
\hline $\mathrm{C} 132-\mathrm{C} 133-\mathrm{C} 134-\mathrm{C} 135$ & $1.80(16)$ \\
\hline $\mathrm{C} 133-\mathrm{C} 134-\mathrm{C} 135-\mathrm{C} 136$ & $-0.70(17)$ \\
\hline $\mathrm{C} 132-\mathrm{C} 131-\mathrm{C} 136-\mathrm{C} 135$ & $-2.80(15)$ \\
\hline $\mathrm{P} 1-\mathrm{C} 131-\mathrm{C} 136-\mathrm{C} 135$ & $173.2(7)$ \\
\hline $\mathrm{C} 134-\mathrm{C} 135-\mathrm{C} 136-\mathrm{C} 131$ & $1.20(14)$ \\
\hline C151-P1—C161-C162 & $-102(3)$ \\
\hline $\mathrm{C} 131-\mathrm{P} 1-\mathrm{C} 161-\mathrm{C} 162$ & $-52(4)$ \\
\hline $\mathrm{C} 121-\mathrm{P} 1-\mathrm{C} 161-\mathrm{C} 162$ & $144(3)$ \\
\hline $\mathrm{C} 141-\mathrm{P} 1-\mathrm{C} 161-\mathrm{C} 162$ & $-112(3)$ \\
\hline NI1-P1-C161-C162 & $22(3)$ \\
\hline $\mathrm{C} 151-\mathrm{P} 1-\mathrm{C} 161-\mathrm{C} 166$ & $72(3)$ \\
\hline $\mathrm{C} 131-\mathrm{P} 1-\mathrm{C} 161-\mathrm{C} 166$ & $123(8)$ \\
\hline $\mathrm{C} 121-\mathrm{P} 1-\mathrm{C} 161-\mathrm{C} 166$ & $-42(3)$ \\
\hline $\mathrm{C} 141-\mathrm{P} 1-\mathrm{C} 161-\mathrm{C} 166$ & $62(3)$ \\
\hline NI1-P1-C161-C166 & $-164(2)$ \\
\hline $\mathrm{C} 166-\mathrm{C} 161-\mathrm{C} 162-\mathrm{C} 163$ & $-1(4)$ \\
\hline $\mathrm{P} 1-\mathrm{C} 161-\mathrm{C} 162-\mathrm{C} 163$ & $175(2)$ \\
\hline $\mathrm{C} 161-\mathrm{C} 162-\mathrm{C} 163-\mathrm{C} 164$ & $7(4)$ \\
\hline $\mathrm{C} 162-\mathrm{C} 163-\mathrm{C} 164-\mathrm{C} 165$ & $-10(5)$ \\
\hline $\mathrm{C} 163-\mathrm{C} 164-\mathrm{C} 165-\mathrm{C} 166$ & $8(5)$ \\
\hline $\mathrm{C} 162-\mathrm{C} 161-\mathrm{C} 166-\mathrm{C} 165$ & $-2(4)$ \\
\hline $\mathrm{P} 1-\mathrm{C} 161-\mathrm{C} 166-\mathrm{C} 165$ & $-176.60(18)$ \\
\hline $\mathrm{C} 164-\mathrm{C} 165-\mathrm{C} 166-\mathrm{C} 161$ & $-2(4)$ \\
\hline C151-P1-C141-C142 & $-127(3)$ \\
\hline $\mathrm{C} 131-\mathrm{P} 1-\mathrm{C} 141-\mathrm{C} 142$ & $-84.0(6)$ \\
\hline $\mathrm{C} 121-\mathrm{P} 1-\mathrm{C} 141-\mathrm{C} 142$ & $24.7(5)$ \\
\hline $\mathrm{C} 161-\mathrm{P} 1-\mathrm{C} 141-\mathrm{C} 142$ & $-76.3(9)$ \\
\hline NI1-P1-C141-C142 & $149.2(4)$ \\
\hline $\mathrm{C} 151-\mathrm{P} 1-\mathrm{C} 141-\mathrm{C} 146$ & $48(2)$ \\
\hline
\end{tabular}




\begin{tabular}{|c|c|}
\hline $\mathrm{C} 131-\mathrm{P} 1-\mathrm{C} 141-\mathrm{C} 146$ & $91.5(5)$ \\
\hline $\mathrm{C} 121-\mathrm{P} 1-\mathrm{C} 141-\mathrm{C} 146$ & $-159.8(4)$ \\
\hline $\mathrm{C} 161-\mathrm{P} 1-\mathrm{C} 141-\mathrm{C} 146$ & $99.2(8)$ \\
\hline NI1-P1-C141-C146 & $-35.3(5)$ \\
\hline $\mathrm{C} 146-\mathrm{C} 141-\mathrm{C} 142-\mathrm{C} 143$ & $-0.2(8)$ \\
\hline $\mathrm{P} 1-\mathrm{C} 141-\mathrm{C} 142-\mathrm{C} 143$ & $175.4(4)$ \\
\hline $\mathrm{C} 141-\mathrm{C} 142-\mathrm{C} 143-\mathrm{C} 144$ & $-0.2(8)$ \\
\hline $\mathrm{C} 142-\mathrm{C} 143-\mathrm{C} 144-\mathrm{C} 145$ & $-0.30(13)$ \\
\hline $\mathrm{C} 143-\mathrm{C} 144-\mathrm{C} 145-\mathrm{C} 146$ & $1.30(14)$ \\
\hline $\mathrm{C} 142-\mathrm{C} 141-\mathrm{C} 146-\mathrm{C} 145$ & $1.1(8)$ \\
\hline $\mathrm{P} 1-\mathrm{C} 141-\mathrm{C} 146-\mathrm{C} 145$ & $-174.7(5)$ \\
\hline $\mathrm{C} 144-\mathrm{C} 145-\mathrm{C} 146-\mathrm{C} 141$ & $-1.70(12)$ \\
\hline C131-P1-C151-C152 & $-24.40(17)$ \\
\hline $\mathrm{C} 121-\mathrm{P} 1-\mathrm{C} 151-\mathrm{C} 152$ & $84.10(16)$ \\
\hline $\mathrm{C} 141-\mathrm{P} 1-\mathrm{C} 151-\mathrm{C} 152$ & $114(4)$ \\
\hline $\mathrm{C} 161-\mathrm{P} 1-\mathrm{C} 151-\mathrm{C} 152$ & $-17.80(18)$ \\
\hline NI1-P1-C151-C152 & $-144.40(14)$ \\
\hline $\mathrm{C} 131-\mathrm{P} 1-\mathrm{C} 151-\mathrm{C} 156$ & $162.60(12)$ \\
\hline $\mathrm{C} 121-\mathrm{P} 1-\mathrm{C} 151-\mathrm{C} 156$ & $-88.90(12)$ \\
\hline $\mathrm{C} 141-\mathrm{P} 1-\mathrm{C} 151-\mathrm{C} 156$ & $-59.20(19)$ \\
\hline $\mathrm{C} 161-\mathrm{P} 1-\mathrm{C} 151-\mathrm{C} 156$ & $169.20(12)$ \\
\hline NI1-P1-C151-C156 & $42.60(14)$ \\
\hline $\mathrm{C} 156-\mathrm{C} 151-\mathrm{C} 152-\mathrm{C} 153$ & $-1(3)$ \\
\hline $\mathrm{P} 1-\mathrm{C} 151-\mathrm{C} 152-\mathrm{C} 153$ & $-172.00(15$ \\
\hline $\mathrm{C} 151-\mathrm{C} 152-\mathrm{C} 153-\mathrm{C} 154$ & $-2(3)$ \\
\hline $\mathrm{C} 152-\mathrm{C} 153-\mathrm{C} 154-\mathrm{C} 155$ & $3(5)$ \\
\hline $\mathrm{C} 153-\mathrm{C} 154-\mathrm{C} 155-\mathrm{C} 156$ & $-3(4)$ \\
\hline $\mathrm{C} 152-\mathrm{C} 151-\mathrm{C} 156-\mathrm{C} 155$ & $1(3)$ \\
\hline $\mathrm{P} 1-\mathrm{C} 151-\mathrm{C} 156-\mathrm{C} 155$ & $173.7(1)$ \\
\hline $\mathrm{C} 154-\mathrm{C} 155-\mathrm{C} 156-\mathrm{C} 151$ & $0(3)$ \\
\hline $\mathrm{C} 23-\mathrm{NI} 2-\mathrm{CL} 2 \mathrm{~A}-\mathrm{CL} 2 \mathrm{~B}$ & $-105.6(8)$ \\
\hline $\mathrm{C} 22-\mathrm{NI} 2-\mathrm{CL} 2 \mathrm{~A}-\mathrm{CL} 2 \mathrm{~B}$ & $-52.5(8)$ \\
\hline $\mathrm{C} 21-\mathrm{NI} 2-\mathrm{CL} 2 \mathrm{~A}-\mathrm{CL} 2 \mathrm{~B}$ & $-78.1(7)$ \\
\hline $\mathrm{P} 2-\mathrm{NI} 2-\mathrm{CL} 2 \mathrm{~A}-\mathrm{CL} 2 \mathrm{~B}$ & $101.2(7)$ \\
\hline $\mathrm{C} 23 \mathrm{~A}-\mathrm{NI} 2-\mathrm{CL} 2 \mathrm{~A}-\mathrm{CL} 2 \mathrm{~B}$ & $-139.9(7)$ \\
\hline $\mathrm{C} 27 \mathrm{~A}-\mathrm{NI} 2-\mathrm{CL} 2 \mathrm{~A}-\mathrm{CL} 2 \mathrm{~B}$ & $-116.1(7)$ \\
\hline C23-NI2-CL2B-CL2A & $125.8(6)$ \\
\hline $\mathrm{C} 22-\mathrm{NI} 2-\mathrm{CL} 2 \mathrm{~B}-\mathrm{CL} 2 \mathrm{~A}$ & $136.3(7)$ \\
\hline $\mathrm{C} 21-\mathrm{NI} 2-\mathrm{CL} 2 \mathrm{~B}-\mathrm{CL} 2 \mathrm{~A}$ & $104.0(7)$ \\
\hline $\mathrm{P} 2-\mathrm{NI} 2-\mathrm{CL} 2 \mathrm{~B}-\mathrm{CL} 2 \mathrm{~A}$ & $-80.6(7)$ \\
\hline $\mathrm{C} 23 \mathrm{~A}-\mathrm{NI} 2-\mathrm{CL} 2 \mathrm{~B}-\mathrm{CL} 2 \mathrm{~A}$ & $51.8(9)$ \\
\hline $\mathrm{C} 27 \mathrm{~A}-\mathrm{NI} 2-\mathrm{CL} 2 \mathrm{~B}-\mathrm{CL} 2 \mathrm{~A}$ & $67.8(7)$ \\
\hline $\mathrm{C} 23-\mathrm{NI} 2-\mathrm{P} 2-\mathrm{C} 291$ & $-7.7(4)$ \\
\hline $\mathrm{C} 22-\mathrm{NI} 2-\mathrm{P} 2-\mathrm{C} 291$ & $-39.6(4)$ \\
\hline $\mathrm{C} 21-\mathrm{NI} 2-\mathrm{P} 2-\mathrm{C} 291$ & $-17.7(5)$ \\
\hline $\mathrm{CL} 2 \mathrm{~A}-\mathrm{NI} 2-\mathrm{P} 2-\mathrm{C} 291$ & $165.0(4)$ \\
\hline CL2B-NI2-P2-C291 & $-179.2(4)$ \\
\hline $\mathrm{C} 23 \mathrm{~A}-\mathrm{NI} 2-\mathrm{P} 2-\mathrm{C} 291$ & $30.2(4)$ \\
\hline $\mathrm{C} 27 \mathrm{~A}-\mathrm{NI} 2-\mathrm{P} 2-\mathrm{C} 291$ & $50.2(4)$ \\
\hline $\mathrm{C} 23-\mathrm{NI} 2-\mathrm{P} 2-\mathrm{C} 271$ & $-118.7(5)$ \\
\hline $\mathrm{C} 22-\mathrm{NI} 2-\mathrm{P} 2-\mathrm{C} 271$ & $-150.5(5)$ \\
\hline $\mathrm{C} 21-\mathrm{NI} 2-\mathrm{P} 2-\mathrm{C} 271$ & $-128.7(6)$ \\
\hline $\mathrm{CL} 2 \mathrm{~A}-\mathrm{NI} 2-\mathrm{P} 2-\mathrm{C} 271$ & $54.0(5)$ \\
\hline $\mathrm{CL} 2 \mathrm{~B}-\mathrm{NI} 2-\mathrm{P} 2-\mathrm{C} 271$ & $69.8(5)$ \\
\hline $\mathrm{C} 23 \mathrm{~A}-\mathrm{NI} 2-\mathrm{P} 2-\mathrm{C} 271$ & $-80.8(5)$ \\
\hline $\mathrm{C} 27 \mathrm{~A}-\mathrm{NI} 2-\mathrm{P} 2-\mathrm{C} 271$ & $-60.8(5)$ \\
\hline $\mathrm{C} 23-\mathrm{NI} 2-\mathrm{P} 2-\mathrm{C} 231$ & $-108.3(6)$ \\
\hline $\mathrm{C} 22-\mathrm{NI} 2-\mathrm{P} 2-\mathrm{C} 231$ & $-140.1(6)$ \\
\hline $\mathrm{C} 21-\mathrm{NI} 2-\mathrm{P} 2-\mathrm{C} 231$ & $-118.3(7)$ \\
\hline $\mathrm{CL} 2 \mathrm{~A}-\mathrm{NI} 2-\mathrm{P} 2-\mathrm{C} 231$ & $64.4(5)$ \\
\hline CL2B-NI2-P2-C231 & $80.2(6)$ \\
\hline $\mathrm{C} 23 \mathrm{~A}-\mathrm{NI} 2-\mathrm{P} 2-\mathrm{C} 231$ & $-70.4(5)$ \\
\hline
\end{tabular}

\begin{tabular}{|c|c|}
\hline $\mathrm{C} 27 \mathrm{~A}-\mathrm{NI} 2-\mathrm{P} 2-\mathrm{C} 231$ & $-50.4(6)$ \\
\hline $\mathrm{C} 23-\mathrm{NI} 2-\mathrm{P} 2-\mathrm{C} 241$ & 115.8 \\
\hline $\mathrm{C} 22-\mathrm{NI} 2-\mathrm{P} 2-\mathrm{C} 241$ & $83.9(3)$ \\
\hline $\mathrm{C} 21-\mathrm{NI} 2-\mathrm{P} 2-\mathrm{C} 241$ & $105.8(5)$ \\
\hline $\mathrm{CL} 2 \mathrm{~A}-\mathrm{NI} 2-\mathrm{P} 2-\mathrm{C} 241$ & $-71.5(3)$ \\
\hline $\mathrm{CL} 2 \mathrm{~B}-\mathrm{NI} 2-\mathrm{P} 2-\mathrm{C} 241$ & $-55.7(3)$ \\
\hline $\mathrm{C} 23 \mathrm{~A}-\mathrm{NI} 2-\mathrm{P} 2-\mathrm{C} 241$ & $153.7(3)$ \\
\hline $\mathrm{C} 27 \mathrm{~A}-\mathrm{NI} 2-\mathrm{P} 2-\mathrm{C} 241$ & $173.7(3)$ \\
\hline $\mathrm{C} 23-\mathrm{NI} 2-\mathrm{P} 2-\mathrm{C} 281$ & $-132.7(9)$ \\
\hline $\mathrm{C} 22-\mathrm{NI} 2-\mathrm{P} 2-\mathrm{C} 281$ & $-164.5(9)$ \\
\hline $\mathrm{C} 21-\mathrm{NI} 2-\mathrm{P} 2-\mathrm{C} 281$ & $-142.7(9)$ \\
\hline $\mathrm{CL} 2 \mathrm{~A}-\mathrm{NI} 2-\mathrm{P} 2-\mathrm{C} 281$ & $40.0(9)$ \\
\hline CL2B-NI2-P2-C281 & $55.9(9)$ \\
\hline $\mathrm{C} 23 \mathrm{~A}-\mathrm{NI} 2-\mathrm{P} 2-\mathrm{C} 281$ & $-94.7(9)$ \\
\hline $\mathrm{C} 27 \mathrm{~A}-\mathrm{NI} 2-\mathrm{P} 2-\mathrm{C} 281$ & $-74.8(9)$ \\
\hline $\mathrm{C} 23-\mathrm{NI} 2-\mathrm{P} 2-\mathrm{C} 251$ & $122.2(6)$ \\
\hline $\mathrm{C} 22-\mathrm{NI} 2-\mathrm{P} 2-\mathrm{C} 251$ & $90.3(6)$ \\
\hline $\mathrm{C} 21-\mathrm{NI} 2-\mathrm{P} 2-\mathrm{C} 251$ & $112.2(7)$ \\
\hline CL2A-NI2-P2-C251 & $-65.1(6)$ \\
\hline CL2B-NI2-P2-C251 & $-49.3(6)$ \\
\hline $\mathrm{C} 23 \mathrm{~A}-\mathrm{NI} 2-\mathrm{P} 2-\mathrm{C} 251$ & $160.1(6)$ \\
\hline $\mathrm{C} 27 \mathrm{~A}-\mathrm{NI} 2-\mathrm{P} 2-\mathrm{C} 251$ & $-180.0(6)$ \\
\hline $\mathrm{C} 23-\mathrm{NI} 2-\mathrm{P} 2-\mathrm{C} 261$ & $-125.7(9)$ \\
\hline $\mathrm{C} 22-\mathrm{NI} 2-\mathrm{P} 2-\mathrm{C} 261$ & $-157.5(9)$ \\
\hline $\mathrm{C} 21-\mathrm{NI} 2-\mathrm{P} 2-\mathrm{C} 261$ & $-135.7(1)$ \\
\hline $\mathrm{CL} 2 \mathrm{~A}-\mathrm{NI} 2-\mathrm{P} 2-\mathrm{C} 261$ & $47.1(9)$ \\
\hline CL2B-NI2-P2-C261 & $62.9(9)$ \\
\hline $\mathrm{C} 23 \mathrm{~A}-\mathrm{NI} 2-\mathrm{P} 2-\mathrm{C} 261$ & $-87.7(9)$ \\
\hline $\mathrm{C} 27 \mathrm{~A}-\mathrm{NI} 2-\mathrm{P} 2-\mathrm{C} 261$ & $-67.8(9)$ \\
\hline $\mathrm{C} 23-\mathrm{NI} 2-\mathrm{P} 2-\mathrm{C} 221$ & $3.4(3)$ \\
\hline $\mathrm{C} 22-\mathrm{NI} 2-\mathrm{P} 2-\mathrm{C} 221$ & $-28.5(3)$ \\
\hline $\mathrm{C} 21-\mathrm{NI} 2-\mathrm{P} 2-\mathrm{C} 221$ & $-6.6(5)$ \\
\hline $\mathrm{CL} 2 \mathrm{~A}-\mathrm{NI} 2-\mathrm{P} 2-\mathrm{C} 221$ & $176.1(2)$ \\
\hline CL2B-NI2-P2-C221 & $-168.1(3)$ \\
\hline $\mathrm{C} 23 \mathrm{~A}-\mathrm{NI} 2-\mathrm{P} 2-\mathrm{C} 221$ & $41.3(3)$ \\
\hline $\mathrm{C} 27 \mathrm{~A}-\mathrm{NI} 2-\mathrm{P} 2-\mathrm{C} 221$ & $61.2(3)$ \\
\hline $\mathrm{C} 213-\mathrm{SI} 2-\mathrm{C} 21-\mathrm{C} 22$ & $-33.4(3)$ \\
\hline $\mathrm{C} 212-\mathrm{SI} 2-\mathrm{C} 21-\mathrm{C} 22$ & $-154.8(3)$ \\
\hline $\mathrm{C} 211-\mathrm{SI} 2-\mathrm{C} 21-\mathrm{C} 22$ & $85.1(3)$ \\
\hline $\mathrm{C} 213-\mathrm{SI} 2-\mathrm{C} 21-\mathrm{C} 27 \mathrm{~A}$ & $160.9(3)$ \\
\hline $\mathrm{C} 212-\mathrm{SI} 2-\mathrm{C} 21-\mathrm{C} 27 \mathrm{~A}$ & $39.5(3)$ \\
\hline $\mathrm{C} 211-\mathrm{SI} 2-\mathrm{C} 21-\mathrm{C} 27 \mathrm{~A}$ & $-80.6(3)$ \\
\hline $\mathrm{C} 213-\mathrm{SI} 2-\mathrm{C} 21-\mathrm{NI} 2$ & $53.4(3)$ \\
\hline $\mathrm{C} 212-\mathrm{SI} 2-\mathrm{C} 21-\mathrm{NI} 2$ & $-68.0(3)$ \\
\hline $\mathrm{C} 211-\mathrm{SI} 2-\mathrm{C} 21-\mathrm{NI} 2$ & $171.8(2)$ \\
\hline $\mathrm{C} 23-\mathrm{NI} 2-\mathrm{C} 21-\mathrm{C} 22$ & $-38.6(2)$ \\
\hline $\mathrm{CL} 2 \mathrm{~A}-\mathrm{NI} 2-\mathrm{C} 21-\mathrm{C} 22$ & $149.5(2)$ \\
\hline $\mathrm{P} 2-\mathrm{NI} 2-\mathrm{C} 21-\mathrm{C} 22$ & $-27.8(5)$ \\
\hline CL2B-NI2-C21-C22 & $133.8(2)$ \\
\hline $\mathrm{C} 23 \mathrm{~A}-\mathrm{NI} 2-\mathrm{C} 21-\mathrm{C} 22$ & $-80.5(2)$ \\
\hline $\mathrm{C} 27 \mathrm{~A}-\mathrm{NI} 2-\mathrm{C} 21-\mathrm{C} 22$ & $-113.3(3)$ \\
\hline $\mathrm{C} 23-\mathrm{NI} 2-\mathrm{C} 21-\mathrm{C} 27 \mathrm{~A}$ & $74.7(2)$ \\
\hline $\mathrm{C} 22-\mathrm{NI} 2-\mathrm{C} 21-\mathrm{C} 27 \mathrm{~A}$ & $113.3(3)$ \\
\hline $\mathrm{CL} 2 \mathrm{~A}-\mathrm{NI} 2-\mathrm{C} 21-\mathrm{C} 27 \mathrm{~A}$ & $-97.26(16)$ \\
\hline $\mathrm{P} 2-\mathrm{NI} 2-\mathrm{C} 21-\mathrm{C} 27 \mathrm{~A}$ & $85.5(4)$ \\
\hline $\mathrm{CL} 2 \mathrm{~B}-\mathrm{NI} 2-\mathrm{C} 21-\mathrm{C} 27 \mathrm{~A}$ & $-112.96(19)$ \\
\hline $\mathrm{C} 23 \mathrm{~A}-\mathrm{NI} 2-\mathrm{C} 21-\mathrm{C} 27 \mathrm{~A}$ & $32.77(17)$ \\
\hline $\mathrm{C} 23-\mathrm{NI} 2-\mathrm{C} 21-\mathrm{SI} 2$ & $-156.1(3)$ \\
\hline $\mathrm{C} 22-\mathrm{NI} 2-\mathrm{C} 21-\mathrm{SI} 2$ & $-117.5(3)$ \\
\hline $\mathrm{CL} 2 \mathrm{~A}-\mathrm{NI} 2-\mathrm{C} 21-\mathrm{SI} 2$ & $32.0(2)$ \\
\hline $\mathrm{P} 2-\mathrm{NI} 2-\mathrm{C} 21-\mathrm{SI} 2$ & $-145.3(2)$ \\
\hline $\mathrm{CL} 2 \mathrm{~B}-\mathrm{NI} 2-\mathrm{C} 21-\mathrm{SI} 2$ & $16.3(2)$ \\
\hline
\end{tabular}




\begin{tabular}{|c|c|}
\hline $\mathrm{C} 23 \mathrm{~A}-\mathrm{NI} 2-\mathrm{C} 21-\mathrm{SI} 2$ & $162.0(3)$ \\
\hline $\mathrm{C} 27 \mathrm{~A}-\mathrm{NI} 2-\mathrm{C} 21-\mathrm{SI} 2$ & $129.2(3)$ \\
\hline $\mathrm{C} 27 \mathrm{~A}-\mathrm{C} 21-\mathrm{C} 22-\mathrm{C} 23$ & $-10.8(3)$ \\
\hline $\mathrm{SI} 2-\mathrm{C} 21-\mathrm{C} 22-\mathrm{C} 23$ & $-178.8(2)$ \\
\hline $\mathrm{NI} 2-\mathrm{C} 21-\mathrm{C} 22-\mathrm{C} 23$ & $60.45(19)$ \\
\hline $\mathrm{C} 27 \mathrm{~A}-\mathrm{C} 21-\mathrm{C} 22-\mathrm{NI} 2$ & $-71.25(18)$ \\
\hline $\mathrm{SI} 2-\mathrm{C} 21-\mathrm{C} 22-\mathrm{NI} 2$ & $120.7(2)$ \\
\hline $\mathrm{C} 21-\mathrm{NI} 2-\mathrm{C} 22-\mathrm{C} 23$ & $-117.8(3)$ \\
\hline $\mathrm{CL} 2 \mathrm{~A}-\mathrm{NI} 2-\mathrm{C} 22-\mathrm{C} 23$ & $-159.91(18)$ \\
\hline $\mathrm{P} 2-\mathrm{NI} 2-\mathrm{C} 22-\mathrm{C} 23$ & $53.7(2)$ \\
\hline $\mathrm{CL} 2 \mathrm{~B}-\mathrm{NI} 2-\mathrm{C} 22-\mathrm{C} 23$ & $-174.7(2)$ \\
\hline $\mathrm{C} 23 \mathrm{~A}-\mathrm{NI} 2-\mathrm{C} 22-\mathrm{C} 23$ & $-39.4(2)$ \\
\hline $\mathrm{C} 27 \mathrm{~A}-\mathrm{NI} 2-\mathrm{C} 22-\mathrm{C} 23$ & $-78.7(2)$ \\
\hline $\mathrm{C} 23-\mathrm{NI} 2-\mathrm{C} 22-\mathrm{C} 21$ & 117.8 \\
\hline $\mathrm{CL} 2 \mathrm{~A}-\mathrm{NI} 2-\mathrm{C} 22-\mathrm{C} 21$ & $-42.1(3)$ \\
\hline $\mathrm{P} 2-\mathrm{NI} 2-\mathrm{C} 22-\mathrm{C} 21$ & 171.50 \\
\hline $\mathrm{CL} 2 \mathrm{~B}-\mathrm{NI} 2-\mathrm{C} 22-\mathrm{C} 21$ & $-56.9(3)$ \\
\hline $\mathrm{C} 23 \mathrm{~A}-\mathrm{NI} 2-\mathrm{C} 22-\mathrm{C} 21$ & $78.4(2)$ \\
\hline $\mathrm{C} 27 \mathrm{~A}-\mathrm{NI} 2-\mathrm{C} 22-\mathrm{C} 21$ & $39.03(19)$ \\
\hline $\mathrm{C} 21-\mathrm{C} 22-\mathrm{C} 23-\mathrm{C} 23 \mathrm{~A}$ & $11.6(3)$ \\
\hline $\mathrm{NI} 2-\mathrm{C} 22-\mathrm{C} 23-\mathrm{C} 23 \mathrm{~A}$ & $74.7(2)$ \\
\hline $\mathrm{C} 21-\mathrm{C} 22-\mathrm{C} 23-\mathrm{NI} 2$ & $-63.1(2)$ \\
\hline $\mathrm{C} 21-\mathrm{NI} 2-\mathrm{C} 23-\mathrm{C} 22$ & $37.75(19)$ \\
\hline $\mathrm{CL} 2 \mathrm{~A}-\mathrm{NI} 2-\mathrm{C} 23-\mathrm{C} 22$ & $67.5(5)$ \\
\hline $\mathrm{P} 2-\mathrm{NI} 2-\mathrm{C} 23-\mathrm{C} 22$ & $-139.52(19)$ \\
\hline $\mathrm{CL} 2 \mathrm{~B}-\mathrm{NI} 2-\mathrm{C} 23-\mathrm{C} 22$ & $14.0(6)$ \\
\hline $\mathrm{C} 23 \mathrm{~A}-\mathrm{NI} 2-\mathrm{C} 23-\mathrm{C} 22$ & $112.6(3)$ \\
\hline $\mathrm{C} 27 \mathrm{~A}-\mathrm{NI} 2-\mathrm{C} 23-\mathrm{C} 22$ & $79.2(2)$ \\
\hline $\mathrm{C} 22-\mathrm{NI} 2-\mathrm{C} 23-\mathrm{C} 23 \mathrm{~A}$ & $-112.6(3)$ \\
\hline $\mathrm{C} 21-\mathrm{NI} 2-\mathrm{C} 23-\mathrm{C} 23 \mathrm{~A}$ & $-74.8(2)$ \\
\hline $\mathrm{CL} 2 \mathrm{~A}-\mathrm{NI} 2-\mathrm{C} 23-\mathrm{C} 23 \mathrm{~A}$ & $-45.0(5)$ \\
\hline $\mathrm{P} 2-\mathrm{NI} 2-\mathrm{C} 23-\mathrm{C} 23 \mathrm{~A}$ & $107.92(17)$ \\
\hline $\mathrm{CL} 2 \mathrm{~B}-\mathrm{NI} 2-\mathrm{C} 23-\mathrm{C} 23 \mathrm{~A}$ & $-98.5(6)$ \\
\hline $\mathrm{C} 27 \mathrm{~A}-\mathrm{NI} 2-\mathrm{C} 23-\mathrm{C} 23 \mathrm{~A}$ & $-33.34(18)$ \\
\hline $\mathrm{C} 23 \mathrm{~A}-\mathrm{C} 24-\mathrm{C} 25-\mathrm{C} 26$ & $-2.3(8)$ \\
\hline $\mathrm{C} 24-\mathrm{C} 25-\mathrm{C} 26-\mathrm{C} 27$ & $1.1(9)$ \\
\hline $\mathrm{C} 25-\mathrm{C} 26-\mathrm{C} 27-\mathrm{C} 27 \mathrm{~A}$ & $1.4(8)$ \\
\hline $\mathrm{C} 291-\mathrm{P} 2-\mathrm{C} 231-\mathrm{C} 236$ & $95.70(14)$ \\
\hline $\mathrm{C} 271-\mathrm{P} 2-\mathrm{C} 231-\mathrm{C} 236$ & $-47(6)$ \\
\hline $\mathrm{C} 241-\mathrm{P} 2-\mathrm{C} 231-\mathrm{C} 236$ & $-5.80(15)$ \\
\hline $\mathrm{C} 281-\mathrm{P} 2-\mathrm{C} 231-\mathrm{C} 236$ & $-56(3)$ \\
\hline $\mathrm{C} 251-\mathrm{P} 2-\mathrm{C} 231-\mathrm{C} 236$ & $-14.40(17)$ \\
\hline $\mathrm{C} 261-\mathrm{P} 2-\mathrm{C} 231-\mathrm{C} 236$ & $-80(3)$ \\
\hline $\mathrm{C} 221-\mathrm{P} 2-\mathrm{C} 231-\mathrm{C} 236$ & $96.80(14)$ \\
\hline $\mathrm{NI} 2-\mathrm{P} 2-\mathrm{C} 231-\mathrm{C} 236$ & $-143.20(12)$ \\
\hline $\mathrm{C} 291-\mathrm{P} 2-\mathrm{C} 231-\mathrm{C} 232$ & $-111.90(13)$ \\
\hline $\mathrm{C} 271-\mathrm{P} 2-\mathrm{C} 231-\mathrm{C} 232$ & $106(7)$ \\
\hline $\mathrm{C} 241-\mathrm{P} 2-\mathrm{C} 231-\mathrm{C} 232$ & $146.60(11)$ \\
\hline $\mathrm{C} 281-\mathrm{P} 2-\mathrm{C} 231-\mathrm{C} 232$ & $96(3)$ \\
\hline $\mathrm{C} 251-\mathrm{P} 2-\mathrm{C} 231-\mathrm{C} 232$ & $138.00(12)$ \\
\hline $\mathrm{C} 261-\mathrm{P} 2-\mathrm{C} 231-\mathrm{C} 232$ & $72(3)$ \\
\hline $\mathrm{C} 221-\mathrm{P} 2-\mathrm{C} 231-\mathrm{C} 232$ & $-110.80(12)$ \\
\hline $\mathrm{NI} 2-\mathrm{P} 2-\mathrm{C} 231-\mathrm{C} 232$ & $9.20(14)$ \\
\hline $\mathrm{C} 236-\mathrm{C} 231-\mathrm{C} 232-\mathrm{C} 233$ & $-30(2)$ \\
\hline $\mathrm{P} 2-\mathrm{C} 231-\mathrm{C} 232-\mathrm{C} 233$ & 177.10 \\
\hline $\mathrm{C} 231-\mathrm{C} 232-\mathrm{C} 233-\mathrm{C} 234$ & $6(3)$ \\
\hline $\mathrm{C} 232-\mathrm{C} 233-\mathrm{C} 234-\mathrm{C} 235$ & $20(3)$ \\
\hline $\mathrm{C} 233-\mathrm{C} 234-\mathrm{C} 235-\mathrm{C} 236$ & $-21(4)$ \\
\hline $\mathrm{C} 232-\mathrm{C} 231-\mathrm{C} 236-\mathrm{C} 235$ & $28(2)$ \\
\hline $\mathrm{P} 2-\mathrm{C} 231-\mathrm{C} 236-\mathrm{C} 235$ & $179.80(14)$ \\
\hline $\mathrm{C} 234-\mathrm{C} 235-\mathrm{C} 236-\mathrm{C} 231$ & $-4(3)$ \\
\hline C291-P2-C261-C262 & $-152(2)$ \\
\hline
\end{tabular}

\begin{tabular}{|c|c|}
\hline $\mathrm{C} 271-\mathrm{P} 2-\mathrm{C} 261-\mathrm{C} 262$ & $-174(9)$ \\
\hline $\mathrm{C} 231-\mathrm{P} 2-\mathrm{C} 261-\mathrm{C} 262$ & $-148(5)$ \\
\hline $\mathrm{C} 241-\mathrm{P} 2-\mathrm{C} 261-\mathrm{C} 262$ & $100(2)$ \\
\hline $\mathrm{C} 281-\mathrm{P} 2-\mathrm{C} 261-\mathrm{C} 262$ & $111(9)$ \\
\hline $\mathrm{C} 251-\mathrm{P} 2-\mathrm{C} 261-\mathrm{C} 262$ & $93(3)$ \\
\hline $\mathrm{C} 221-\mathrm{P} 2-\mathrm{C} 261-\mathrm{C} 262$ & $-151(2)$ \\
\hline NI2-P2-C261-C262 & $-24(3)$ \\
\hline $\mathrm{C} 291-\mathrm{P} 2-\mathrm{C} 261-\mathrm{C} 266$ & $28(2)$ \\
\hline $\mathrm{C} 271-\mathrm{P} 2-\mathrm{C} 261-\mathrm{C} 266$ & $7(5)$ \\
\hline $\mathrm{C} 231-\mathrm{P} 2-\mathrm{C} 261-\mathrm{C} 266$ & $32.40(18)$ \\
\hline $\mathrm{C} 241-\mathrm{P} 2-\mathrm{C} 261-\mathrm{C} 266$ & $-79(2)$ \\
\hline $\mathrm{C} 281-\mathrm{P} 2-\mathrm{C} 261-\mathrm{C} 266$ & $-68(6)$ \\
\hline $\mathrm{C} 251-\mathrm{P} 2-\mathrm{C} 261-\mathrm{C} 266$ & $-87(2)$ \\
\hline $\mathrm{C} 221-\mathrm{P} 2-\mathrm{C} 261-\mathrm{C} 266$ & $29(3)$ \\
\hline $\mathrm{NI} 2-\mathrm{P} 2-\mathrm{C} 261-\mathrm{C} 266$ & $156(2)$ \\
\hline $\mathrm{C} 266-\mathrm{C} 261-\mathrm{C} 262-\mathrm{C} 263$ & $-1(4)$ \\
\hline $\mathrm{P} 2-\mathrm{C} 261-\mathrm{C} 262-\mathrm{C} 263$ & $180(2)$ \\
\hline $\mathrm{C} 261-\mathrm{C} 262-\mathrm{C} 263-\mathrm{C} 264$ & $2(4)$ \\
\hline $\mathrm{C} 262-\mathrm{C} 263-\mathrm{C} 264-\mathrm{C} 265$ & $-7(4)$ \\
\hline $\mathrm{C} 263-\mathrm{C} 264-\mathrm{C} 265-\mathrm{C} 266$ & $10(4)$ \\
\hline $\mathrm{C} 262-\mathrm{C} 261-\mathrm{C} 266-\mathrm{C} 265$ & $4(4)$ \\
\hline $\mathrm{P} 2-\mathrm{C} 261-\mathrm{C} 266-\mathrm{C} 265$ & $-176(2)$ \\
\hline $\mathrm{C} 264-\mathrm{C} 265-\mathrm{C} 266-\mathrm{C} 261$ & $-9(4)$ \\
\hline $\mathrm{C} 291-\mathrm{P} 2-\mathrm{C} 271-\mathrm{C} 272$ & $51.00(14)$ \\
\hline $\mathrm{C} 231-\mathrm{P} 2-\mathrm{C} 271-\mathrm{C} 272$ & $89(6)$ \\
\hline $\mathrm{C} 241-\mathrm{P} 2-\mathrm{C} 271-\mathrm{C} 272$ & $-52.60(13)$ \\
\hline $\mathrm{C} 281-\mathrm{P} 2-\mathrm{C} 271-\mathrm{C} 272$ & $-107(4)$ \\
\hline $\mathrm{C} 251-\mathrm{P} 2-\mathrm{C} 271-\mathrm{C} 272$ & $-60.70(15)$ \\
\hline $\mathrm{C} 261-\mathrm{P} 2-\mathrm{C} 271-\mathrm{C} 272$ & $-150(7)$ \\
\hline $\mathrm{C} 221-\mathrm{P} 2-\mathrm{C} 271-\mathrm{C} 272$ & $50.90(14)$ \\
\hline $\mathrm{NI} 2-\mathrm{P} 2-\mathrm{C} 271-\mathrm{C} 272$ & $176.90(11)$ \\
\hline $\mathrm{C} 291-\mathrm{P} 2-\mathrm{C} 271-\mathrm{C} 276$ & $-81.00(11)$ \\
\hline $\mathrm{C} 231-\mathrm{P} 2-\mathrm{C} 271-\mathrm{C} 276$ & $-43(6)$ \\
\hline $\mathrm{C} 241-\mathrm{P} 2-\mathrm{C} 271-\mathrm{C} 276$ & $175.4(9)$ \\
\hline $\mathrm{C} 281-\mathrm{P} 2-\mathrm{C} 271-\mathrm{C} 276$ & $121(4)$ \\
\hline $\mathrm{C} 251-\mathrm{P} 2-\mathrm{C} 271-\mathrm{C} 276$ & $167.30(11)$ \\
\hline $\mathrm{C} 261-\mathrm{P} 2-\mathrm{C} 271-\mathrm{C} 276$ & $78(7)$ \\
\hline $\mathrm{C} 221-\mathrm{P} 2-\mathrm{C} 271-\mathrm{C} 276$ & $-81.1(1)$ \\
\hline $\mathrm{NI} 2-\mathrm{P} 2-\mathrm{C} 271-\mathrm{C} 276$ & $44.90(11)$ \\
\hline $\mathrm{C} 276-\mathrm{C} 271-\mathrm{C} 272-\mathrm{C} 273$ & $-42(2)$ \\
\hline $\mathrm{P} 2-\mathrm{C} 271-\mathrm{C} 272-\mathrm{C} 273$ & $-176.10(16)$ \\
\hline $\mathrm{C} 271-\mathrm{C} 272-\mathrm{C} 273-\mathrm{C} 274$ & $14(3)$ \\
\hline $\mathrm{C} 272-\mathrm{C} 273-\mathrm{C} 274-\mathrm{C} 275$ & $21(3)$ \\
\hline $\mathrm{C} 273-\mathrm{C} 274-\mathrm{C} 275-\mathrm{C} 276$ & $-27(4)$ \\
\hline $\mathrm{C} 272-\mathrm{C} 271-\mathrm{C} 276-\mathrm{C} 275$ & $32.30(18)$ \\
\hline $\mathrm{P} 2-\mathrm{C} 271-\mathrm{C} 276-\mathrm{C} 275$ & $174.20(12)$ \\
\hline $\mathrm{C} 274-\mathrm{C} 275-\mathrm{C} 276-\mathrm{C} 271$ & $-2(3)$ \\
\hline $\mathrm{C} 291-\mathrm{P} 2-\mathrm{C} 281-\mathrm{C} 282$ & $-170(2)$ \\
\hline $\mathrm{C} 271-\mathrm{P} 2-\mathrm{C} 281-\mathrm{C} 282$ & $-147(5)$ \\
\hline $\mathrm{C} 231-\mathrm{P} 2-\mathrm{C} 281-\mathrm{C} 282$ & $-140(5)$ \\
\hline $\mathrm{C} 241-\mathrm{P} 2-\mathrm{C} 281-\mathrm{C} 282$ & $85(2)$ \\
\hline $\mathrm{C} 251-\mathrm{P} 2-\mathrm{C} 281-\mathrm{C} 282$ & $78(2)$ \\
\hline $\mathrm{C} 261-\mathrm{P} 2-\mathrm{C} 281-\mathrm{C} 282$ & $-84(7)$ \\
\hline $\mathrm{C} 221-\mathrm{P} 2-\mathrm{C} 281-\mathrm{C} 282$ & $-171(2)$ \\
\hline NI2-P2-C281-C282 & $-37(3)$ \\
\hline $\mathrm{C} 291-\mathrm{P} 2-\mathrm{C} 281-\mathrm{C} 286$ & $55(2)$ \\
\hline $\mathrm{C} 271-\mathrm{P} 2-\mathrm{C} 281-\mathrm{C} 286$ & $78(4)$ \\
\hline $\mathrm{C} 231-\mathrm{P} 2-\mathrm{C} 281-\mathrm{C} 286$ & $84(3)$ \\
\hline $\mathrm{C} 241-\mathrm{P} 2-\mathrm{C} 281-\mathrm{C} 286$ & $-50.10(18)$ \\
\hline $\mathrm{C} 251-\mathrm{P} 2-\mathrm{C} 281-\mathrm{C} 286$ & $-57.70(19)$ \\
\hline $\mathrm{C} 261-\mathrm{P} 2-\mathrm{C} 281-\mathrm{C} 286$ & $141(8)$ \\
\hline $\mathrm{C} 221-\mathrm{P} 2-\mathrm{C} 281-\mathrm{C} 286$ & $54(2)$ \\
\hline
\end{tabular}




\begin{tabular}{|c|c|}
\hline $\mathrm{NI} 2-\mathrm{P} 2-\mathrm{C} 281-\mathrm{C} 286$ & $-172.30(15)$ \\
\hline $\mathrm{C} 286-\mathrm{C} 281-\mathrm{C} 282-\mathrm{C} 283$ & $-41(3)$ \\
\hline $\mathrm{P} 2-\mathrm{C} 281-\mathrm{C} 282-\mathrm{C} 283$ & $-179(2)$ \\
\hline $\mathrm{C} 281-\mathrm{C} 282-\mathrm{C} 283-\mathrm{C} 284$ & $2(4)$ \\
\hline $\mathrm{C} 282-\mathrm{C} 283-\mathrm{C} 284-\mathrm{C} 285$ & $23(3)$ \\
\hline $\mathrm{C} 283-\mathrm{C} 284-\mathrm{C} 285-\mathrm{C} 286$ & $-6(3)$ \\
\hline $\mathrm{C} 282-\mathrm{C} 281-\mathrm{C} 286-\mathrm{C} 285$ & $55(3)$ \\
\hline $\mathrm{P} 2-\mathrm{C} 281-\mathrm{C} 286-\mathrm{C} 285$ & $-157.20(19)$ \\
\hline $\mathrm{C} 284-\mathrm{C} 285-\mathrm{C} 286-\mathrm{C} 281$ & $-36(3)$ \\
\hline $\mathrm{C} 291-\mathrm{P} 2-\mathrm{C} 241-\mathrm{C} 246$ & $-13.4(8)$ \\
\hline $\mathrm{C} 271-\mathrm{P} 2-\mathrm{C} 241-\mathrm{C} 246$ & $82.3(8)$ \\
\hline $\mathrm{C} 231-\mathrm{P} 2-\mathrm{C} 241-\mathrm{C} 246$ & $76.1(9)$ \\
\hline $\mathrm{C} 281-\mathrm{P} 2-\mathrm{C} 241-\mathrm{C} 246$ & $93.0(9)$ \\
\hline $\mathrm{C} 251-\mathrm{P} 2-\mathrm{C} 241-\mathrm{C} 246$ & $165(4)$ \\
\hline $\mathrm{C} 261-\mathrm{P} 2-\mathrm{C} 241-\mathrm{C} 246$ & $94.8(8)$ \\
\hline $\mathrm{C} 221-\mathrm{P} 2-\mathrm{C} 241-\mathrm{C} 246$ & $-25.4(7)$ \\
\hline $\mathrm{NI} 2-\mathrm{P} 2-\mathrm{C} 241-\mathrm{C} 246$ & $-145.5(5)$ \\
\hline $\mathrm{C} 291-\mathrm{P} 2-\mathrm{C} 241-\mathrm{C} 242$ & $169.1(7)$ \\
\hline $\mathrm{C} 271-\mathrm{P} 2-\mathrm{C} 241-\mathrm{C} 242$ & $-95.2(7)$ \\
\hline $\mathrm{C} 231-\mathrm{P} 2-\mathrm{C} 241-\mathrm{C} 242$ & $-101.4(8)$ \\
\hline $\mathrm{C} 281-\mathrm{P} 2-\mathrm{C} 241-\mathrm{C} 242$ & $-84.5(9)$ \\
\hline $\mathrm{C} 251-\mathrm{P} 2-\mathrm{C} 241-\mathrm{C} 242$ & $-12(3)$ \\
\hline $\mathrm{C} 261-\mathrm{P} 2-\mathrm{C} 241-\mathrm{C} 242$ & $-82.7(8)$ \\
\hline $\mathrm{C} 221-\mathrm{P} 2-\mathrm{C} 241-\mathrm{C} 242$ & $157.1(5)$ \\
\hline $\mathrm{NI} 2-\mathrm{P} 2-\mathrm{C} 241-\mathrm{C} 242$ & $37.0(6)$ \\
\hline $\mathrm{C} 246-\mathrm{C} 241-\mathrm{C} 242-\mathrm{C} 243$ & $-1.6(1)$ \\
\hline $\mathrm{P} 2-\mathrm{C} 241-\mathrm{C} 242-\mathrm{C} 243$ & $176.1(5)$ \\
\hline $\mathrm{C} 241-\mathrm{C} 242-\mathrm{C} 243-\mathrm{C} 244$ & $0.00(12)$ \\
\hline $\mathrm{C} 242-\mathrm{C} 243-\mathrm{C} 244-\mathrm{C} 245$ & $2.90(17)$ \\
\hline $\mathrm{C} 243-\mathrm{C} 244-\mathrm{C} 245-\mathrm{C} 246$ & $-4.10(17)$ \\
\hline $\mathrm{C} 242-\mathrm{C} 241-\mathrm{C} 246-\mathrm{C} 245$ & $0.4(1)$ \\
\hline $\mathrm{P} 2-\mathrm{C} 241-\mathrm{C} 246-\mathrm{C} 245$ & $-177.0(6)$ \\
\hline $\mathrm{C} 244-\mathrm{C} 245-\mathrm{C} 246-\mathrm{C} 241$ & $2.50(13)$ \\
\hline $\mathrm{C} 291-\mathrm{P} 2-\mathrm{C} 251-\mathrm{C} 256$ & $92.30(15)$ \\
\hline $\mathrm{C} 271-\mathrm{P} 2-\mathrm{C} 251-\mathrm{C} 256$ & $-170.00(13)$ \\
\hline $\mathrm{C} 231-\mathrm{P} 2-\mathrm{C} 251-\mathrm{C} 256$ & $-175.00(13)$ \\
\hline $\mathrm{C} 241-\mathrm{P} 2-\mathrm{C} 251-\mathrm{C} 256$ & $91(4)$ \\
\hline $\mathrm{C} 281-\mathrm{P} 2-\mathrm{C} 251-\mathrm{C} 256$ & $-160.60(15)$ \\
\hline $\mathrm{C} 261-\mathrm{P} 2-\mathrm{C} 251-\mathrm{C} 256$ & $-157.60(14)$ \\
\hline $\mathrm{C} 221-\mathrm{P} 2-\mathrm{C} 251-\mathrm{C} 256$ & $79.90(14)$ \\
\hline NI2-P2-C251-C256 & $-42.40(16)$ \\
\hline $\mathrm{C} 291-\mathrm{P} 2-\mathrm{C} 251-\mathrm{C} 252$ & $-76.40(18)$ \\
\hline $\mathrm{C} 271-\mathrm{P} 2-\mathrm{C} 251-\mathrm{C} 252$ & $21.20(18)$ \\
\hline $\mathrm{C} 231-\mathrm{P} 2-\mathrm{C} 251-\mathrm{C} 252$ & $16(2)$ \\
\hline $\mathrm{C} 241-\mathrm{P} 2-\mathrm{C} 251-\mathrm{C} 252$ & $-78(3)$ \\
\hline $\mathrm{C} 281-\mathrm{P} 2-\mathrm{C} 251-\mathrm{C} 252$ & $30.70(18)$ \\
\hline $\mathrm{C} 261-\mathrm{P} 2-\mathrm{C} 251-\mathrm{C} 252$ & $33.60(18)$ \\
\hline $\mathrm{C} 221-\mathrm{P} 2-\mathrm{C} 251-\mathrm{C} 252$ & $-88.90(16)$ \\
\hline NI2-P2-C251-C252 & $148.80(14)$ \\
\hline $\mathrm{C} 256-\mathrm{C} 251-\mathrm{C} 252-\mathrm{C} 253$ & $0(2)$ \\
\hline $\mathrm{P} 2-\mathrm{C} 251-\mathrm{C} 252-\mathrm{C} 253$ & $168.50(12)$ \\
\hline $\mathrm{C} 251-\mathrm{C} 252-\mathrm{C} 253-\mathrm{C} 254$ & $0(2)$ \\
\hline $\mathrm{C} 252-\mathrm{C} 253-\mathrm{C} 254-\mathrm{C} 255$ & $0(2)$ \\
\hline $\mathrm{C} 253-\mathrm{C} 254-\mathrm{C} 255-\mathrm{C} 256$ & $0(2)$ \\
\hline $\mathrm{C} 252-\mathrm{C} 251-\mathrm{C} 256-\mathrm{C} 255$ & $0(2)$ \\
\hline $\mathrm{P} 2-\mathrm{C} 251-\mathrm{C} 256-\mathrm{C} 255$ & $-169.3(9)$ \\
\hline $\mathrm{C} 254-\mathrm{C} 255-\mathrm{C} 256-\mathrm{C} 251$ & $0.20(19)$ \\
\hline $\mathrm{C} 291-\mathrm{P} 2-\mathrm{C} 221-\mathrm{C} 226$ & $-162(4)$ \\
\hline $\mathrm{C} 271-\mathrm{P} 2-\mathrm{C} 221-\mathrm{C} 226$ & $-161.8(9)$ \\
\hline $\mathrm{C} 231-\mathrm{P} 2-\mathrm{C} 221-\mathrm{C} 226$ & $-167.5(9)$ \\
\hline $\mathrm{C} 241-\mathrm{P} 2-\mathrm{C} 221-\mathrm{C} 226$ & $-53.8(8)$ \\
\hline $\mathrm{C} 281-\mathrm{P} 2-\mathrm{C} 221-\mathrm{C} 226$ & $-156.30(11$ \\
\hline
\end{tabular}

$\mathrm{C} 251-\mathrm{P} 2-\mathrm{C} 221-\mathrm{C} 226$ $\mathrm{C} 261-\mathrm{P} 2-\mathrm{C} 221-\mathrm{C} 226$ $\mathrm{NI} 2-\mathrm{P} 2-\mathrm{C} 221-\mathrm{C} 226$ $\mathrm{C} 291-\mathrm{P} 2-\mathrm{C} 221-\mathrm{C} 222$ $\mathrm{C} 271-\mathrm{P} 2-\mathrm{C} 221-\mathrm{C} 222$ $\mathrm{C} 231-\mathrm{P} 2-\mathrm{C} 221-\mathrm{C} 222$ $\mathrm{C} 241-\mathrm{P} 2-\mathrm{C} 221-\mathrm{C} 222$ $\mathrm{C} 281-\mathrm{P} 2-\mathrm{C} 221-\mathrm{C} 222$ $\mathrm{C} 251-\mathrm{P} 2-\mathrm{C} 221-\mathrm{C} 222$ $\mathrm{C} 261-\mathrm{P} 2-\mathrm{C} 221-\mathrm{C} 222$

$\mathrm{NI} 2-\mathrm{P} 2-\mathrm{C} 221-\mathrm{C} 222$ $\mathrm{C} 226-\mathrm{C} 221-\mathrm{C} 222-\mathrm{C} 223$ $\mathrm{P} 2-\mathrm{C} 221-\mathrm{C} 222-\mathrm{C} 223$ $\mathrm{C} 221-\mathrm{C} 222-\mathrm{C} 223-\mathrm{C} 224$ $\mathrm{C} 222$ - $\mathrm{C} 223-\mathrm{C} 224-\mathrm{C} 225$ $\mathrm{C} 223$ - $\mathrm{C} 224-\mathrm{C} 225-\mathrm{C} 226$ $\mathrm{C} 222-\mathrm{C} 221-\mathrm{C} 226-\mathrm{C} 225$ $\mathrm{P} 2-\mathrm{C} 221-\mathrm{C} 226-\mathrm{C} 225$ $\mathrm{C} 224-\mathrm{C} 225-\mathrm{C} 226-\mathrm{C} 221$ $\mathrm{C} 271-\mathrm{P} 2-\mathrm{C} 291-\mathrm{C} 296$ $\mathrm{C} 231-\mathrm{P} 2-\mathrm{C} 291-\mathrm{C} 296$ $\mathrm{C} 241-\mathrm{P} 2-\mathrm{C} 291-\mathrm{C} 296$ $\mathrm{C} 281-\mathrm{P} 2-\mathrm{C} 291-\mathrm{C} 296$ $\mathrm{C} 251-\mathrm{P} 2-\mathrm{C} 291-\mathrm{C} 296$ $\mathrm{C} 261-\mathrm{P} 2-\mathrm{C} 291-\mathrm{C} 296$ $\mathrm{C} 221-\mathrm{P} 2-\mathrm{C} 291-\mathrm{C} 296$ NI2-P2-C291-C296 $\mathrm{C} 271-\mathrm{P} 2-\mathrm{C} 291-\mathrm{C} 292$ $\mathrm{C} 231-\mathrm{P} 2-\mathrm{C} 291-\mathrm{C} 292$ $\mathrm{C} 241-\mathrm{P} 2-\mathrm{C} 291-\mathrm{C} 292$ $\mathrm{C} 281-\mathrm{P} 2-\mathrm{C} 291-\mathrm{C} 292$ $\mathrm{C} 251-\mathrm{P} 2-\mathrm{C} 291-\mathrm{C} 292$ $\mathrm{C} 261-\mathrm{P} 2-\mathrm{C} 291-\mathrm{C} 292$ $\mathrm{C} 221-\mathrm{P} 2-\mathrm{C} 291-\mathrm{C} 292$

$\mathrm{NI} 2-\mathrm{P} 2-\mathrm{C} 291-\mathrm{C} 292$ $\mathrm{C} 296$ - C291-C292-C293 $\mathrm{P} 2-\mathrm{C} 291-\mathrm{C} 292-\mathrm{C} 293$ $\mathrm{C} 291-\mathrm{C} 292-\mathrm{C} 293-\mathrm{C} 294$ $\mathrm{C} 292-\mathrm{C} 293-\mathrm{C} 294-\mathrm{C} 295$ $\mathrm{C} 293$ - $\mathrm{C} 294-\mathrm{C} 295-\mathrm{C} 296$ $\mathrm{C} 292-\mathrm{C} 291-\mathrm{C} 296-\mathrm{C} 295$ $\mathrm{P} 2-\mathrm{C} 291-\mathrm{C} 296-\mathrm{C} 295$ $\mathrm{C} 294-\mathrm{C} 295-\mathrm{C} 296-\mathrm{C} 291$ $\mathrm{C} 15-\mathrm{C} 14-\mathrm{C} 13 \mathrm{~A}-\mathrm{C} 17 \mathrm{~A}$

$\mathrm{C} 15-\mathrm{C} 14-\mathrm{C} 13 \mathrm{~A}-\mathrm{C} 13$

$\mathrm{C} 15-\mathrm{C} 14-\mathrm{C} 13 \mathrm{~A}-\mathrm{NI} 1$

$\mathrm{C} 12-\mathrm{C} 13-\mathrm{C} 13 \mathrm{~A}-\mathrm{C} 14$

$\mathrm{NI1}-\mathrm{C} 13-\mathrm{C} 13 \mathrm{~A}-\mathrm{C} 14$

$\mathrm{C} 12-\mathrm{C} 13-\mathrm{C} 13 \mathrm{~A}-\mathrm{C} 17 \mathrm{~A}$

$\mathrm{NI} 1-\mathrm{C} 13-\mathrm{C} 13 \mathrm{~A}-\mathrm{C} 17 \mathrm{~A}$

$\mathrm{C} 12-\mathrm{C} 13-\mathrm{C} 13 \mathrm{~A}-\mathrm{NI} 1$

$\mathrm{C} 13-\mathrm{NI} 1-\mathrm{C} 13 \mathrm{~A}-\mathrm{C} 14$

$\mathrm{C} 12-\mathrm{NI} 1-\mathrm{C} 13 \mathrm{~A}-\mathrm{C} 14$

$\mathrm{C} 11-\mathrm{NI} 1-\mathrm{C} 13 \mathrm{~A}-\mathrm{C} 14$

CL1-NI1-C13A-C14

P1-NI1-C13A-C14

C17A-NI1-C13A-C14

C13-NI1-C13A-C17A

C12-NI1-C13A-C17A

C11-NI1-C13A-C17A

CL1-NI1-C13A-C17A

$\mathrm{P} 1-\mathrm{NI} 1-\mathrm{C} 13 \mathrm{~A}-\mathrm{C} 17 \mathrm{~A}$

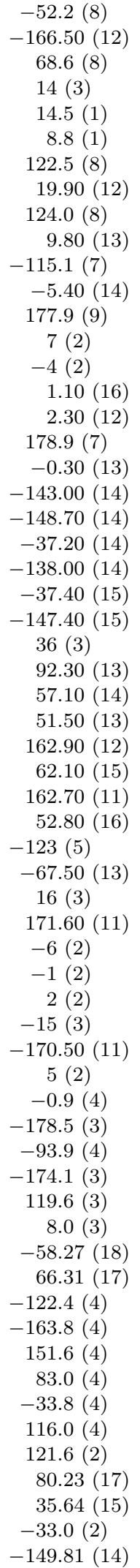




\begin{tabular}{|c|c|c|c|}
\hline $\mathrm{C} 12-\mathrm{NI} 1-\mathrm{C} 13 \mathrm{~A}-\mathrm{C} 13$ & $-41.36(16)$ & $\mathrm{CL} 2 \mathrm{~B}-\mathrm{NI} 2-\mathrm{C} 23 \mathrm{~A}-\mathrm{C} 27 \mathrm{~A}$ & $27.3(3)$ \\
\hline $\mathrm{C} 11-\mathrm{NI} 1-\mathrm{C} 13 \mathrm{~A}-\mathrm{C} 13$ & $-85.95(17)$ & $\mathrm{C} 23-\mathrm{NI} 2-\mathrm{C} 23 \mathrm{~A}-\mathrm{C} 24$ & $124.0(5)$ \\
\hline CL1-NI1-C13A-C13 & $-154.56(14)$ & $\mathrm{C} 22-\mathrm{NI} 2-\mathrm{C} 23 \mathrm{~A}-\mathrm{C} 24$ & $165.9(4)$ \\
\hline P1-NI1-C13A-C13 & $88.60(15)$ & $\mathrm{C} 21-\mathrm{NI} 2-\mathrm{C} 23 \mathrm{~A}-\mathrm{C} 24$ & $-149.6(4)$ \\
\hline $\mathrm{C} 17 \mathrm{~A}-\mathrm{NI} 1-\mathrm{C} 13 \mathrm{~A}-\mathrm{C} 13$ & $-121.6(2)$ & $\mathrm{CL} 2 \mathrm{~A}-\mathrm{NI} 2-\mathrm{C} 23 \mathrm{~A}-\mathrm{C} 24$ & $-70.8(4)$ \\
\hline $\mathrm{C} 16-\mathrm{C} 17-\mathrm{C} 17 \mathrm{~A}-\mathrm{C} 13 \mathrm{~A}$ & $2.8(4)$ & $\mathrm{P} 2-\mathrm{NI} 2-\mathrm{C} 23 \mathrm{~A}-\mathrm{C} 24$ & $42.6(4)$ \\
\hline $\mathrm{C} 16-\mathrm{C} 17-\mathrm{C} 17 \mathrm{~A}-\mathrm{C} 11$ & $-179.9(3)$ & $\mathrm{CL} 2 \mathrm{~B}-\mathrm{NI} 2-\mathrm{C} 23 \mathrm{~A}-\mathrm{C} 24$ & $-87.1(5)$ \\
\hline $\mathrm{C} 16-\mathrm{C} 17-\mathrm{C} 17 \mathrm{~A}-\mathrm{NI} 1$ & $93.0(3)$ & $\mathrm{C} 27 \mathrm{~A}-\mathrm{NI} 2-\mathrm{C} 23 \mathrm{~A}-\mathrm{C} 24$ & $-114.4(5)$ \\
\hline $\mathrm{C} 14-\mathrm{C} 13 \mathrm{~A}-\mathrm{C} 17 \mathrm{~A}-\mathrm{C} 17$ & $-1.8(4)$ & $\mathrm{C} 22-\mathrm{NI} 2-\mathrm{C} 23 \mathrm{~A}-\mathrm{C} 23$ & $41.93(19)$ \\
\hline $\mathrm{C} 13-\mathrm{C} 13 \mathrm{~A}-\mathrm{C} 17 \mathrm{~A}-\mathrm{C} 17$ & $176.3(2)$ & $\mathrm{C} 21-\mathrm{NI} 2-\mathrm{C} 23 \mathrm{~A}-\mathrm{C} 23$ & $86.4(2)$ \\
\hline $\mathrm{NI} 1-\mathrm{C} 13 \mathrm{~A}-\mathrm{C} 17 \mathrm{~A}-\mathrm{C} 17$ & $125.3(2)$ & $\mathrm{CL} 2 \mathrm{~A}-\mathrm{NI} 2-\mathrm{C} 23 \mathrm{~A}-\mathrm{C} 23$ & $165.21(17)$ \\
\hline $\mathrm{C} 14-\mathrm{C} 13 \mathrm{~A}-\mathrm{C} 17 \mathrm{~A}-\mathrm{C} 11$ & $-179.7(2)$ & $\mathrm{P} 2-\mathrm{NI} 2-\mathrm{C} 23 \mathrm{~A}-\mathrm{C} 23$ & $-81.39(19)$ \\
\hline $\mathrm{C} 13-\mathrm{C} 13 \mathrm{~A}-\mathrm{C} 17 \mathrm{~A}-\mathrm{C} 11$ & $-1.5(3)$ & $\mathrm{CL} 2 \mathrm{~B}-\mathrm{NI} 2-\mathrm{C} 23 \mathrm{~A}-\mathrm{C} 23$ & $148.9(3)$ \\
\hline NI1-C13A-C17A-C11 & $-52.56(15)$ & $\mathrm{C} 27 \mathrm{~A}-\mathrm{NI} 2-\mathrm{C} 23 \mathrm{~A}-\mathrm{C} 23$ & $121.6(3)$ \\
\hline $\mathrm{C} 14-\mathrm{C} 13 \mathrm{~A}-\mathrm{C} 17 \mathrm{~A}-\mathrm{NI} 1$ & $-127.2(3)$ & $\mathrm{C} 26-\mathrm{C} 27-\mathrm{C} 27 \mathrm{~A}-\mathrm{C} 23 \mathrm{~A}$ & $-2.6(5)$ \\
\hline $\mathrm{C} 13-\mathrm{C} 13 \mathrm{~A}-\mathrm{C} 17 \mathrm{~A}-\mathrm{NI} 1$ & $51.02(16)$ & $\mathrm{C} 26-\mathrm{C} 27-\mathrm{C} 27 \mathrm{~A}-\mathrm{C} 21$ & $180.0(4)$ \\
\hline $\mathrm{C} 12-\mathrm{C} 11-\mathrm{C} 17 \mathrm{~A}-\mathrm{C} 17$ & $177.1(3)$ & $\mathrm{C} 26-\mathrm{C} 27-\mathrm{C} 27 \mathrm{~A}-\mathrm{NI} 2$ & $-92.8(5)$ \\
\hline $\mathrm{S} 11-\mathrm{C} 11-\mathrm{C} 17 \mathrm{~A}-\mathrm{C} 17$ & $8.8(4)$ & $\mathrm{C} 24-\mathrm{C} 23 \mathrm{~A}-\mathrm{C} 27 \mathrm{~A}-\mathrm{C} 27$ & $1.4(4)$ \\
\hline $\mathrm{NI} 1-\mathrm{C} 11-\mathrm{C} 17 \mathrm{~A}-\mathrm{C} 17$ & $-119.9(3)$ & $\mathrm{C} 23-\mathrm{C} 23 \mathrm{~A}-\mathrm{C} 27 \mathrm{~A}-\mathrm{C} 27$ & $-176.9(3)$ \\
\hline $\mathrm{C} 12-\mathrm{C} 11-\mathrm{C} 17 \mathrm{~A}-\mathrm{C} 13 \mathrm{~A}$ & $-5.3(3)$ & $\mathrm{NI} 2-\mathrm{C} 23 \mathrm{~A}-\mathrm{C} 27 \mathrm{~A}-\mathrm{C} 27$ & $-126.6(3)$ \\
\hline $\mathrm{SI} 1-\mathrm{C} 11-\mathrm{C} 17 \mathrm{~A}-\mathrm{C} 13 \mathrm{~A}$ & $-173.63(17)$ & $\mathrm{C} 24-\mathrm{C} 23 \mathrm{~A}-\mathrm{C} 27 \mathrm{~A}-\mathrm{C} 21$ & $179.4(3)$ \\
\hline $\mathrm{N} 11-\mathrm{C} 11-\mathrm{C} 17 \mathrm{~A}-\mathrm{C} 13 \mathrm{~A}$ & $57.63(17)$ & $\mathrm{C} 23-\mathrm{C} 23 \mathrm{~A}-\mathrm{C} 27 \mathrm{~A}-\mathrm{C} 21$ & $1.1(3)$ \\
\hline $\mathrm{C} 12-\mathrm{C} 11-\mathrm{C} 17 \mathrm{~A}-\mathrm{NI} 1$ & $-62.97(15)$ & $\mathrm{NI} 2-\mathrm{C} 23 \mathrm{~A}-\mathrm{C} 27 \mathrm{~A}-\mathrm{C} 21$ & $51.41(17)$ \\
\hline SI1-C11-C17A-NI1 & $128.7(2)$ & $\mathrm{C} 24-\mathrm{C} 23 \mathrm{~A}-\mathrm{C} 27 \mathrm{~A}-\mathrm{NI} 2$ & $128.0(3)$ \\
\hline $\mathrm{C} 13-\mathrm{N} 11-\mathrm{C} 17 \mathrm{~A}-\mathrm{C} 17$ & $-150.1(3)$ & $\mathrm{C} 23-\mathrm{C} 23 \mathrm{~A}-\mathrm{C} 27 \mathrm{~A}-\mathrm{NI} 2$ & $-50.33(18)$ \\
\hline $\mathrm{C} 12-\mathrm{NI} 1-\mathrm{C} 17 \mathrm{~A}-\mathrm{C} 17$ & $165.0(3)$ & $\mathrm{C} 22-\mathrm{C} 21-\mathrm{C} 27 \mathrm{~A}-\mathrm{C} 27$ & $-176.4(3)$ \\
\hline $\mathrm{C} 11-\mathrm{NI} 1-\mathrm{C} 17 \mathrm{~A}-\mathrm{C} 17$ & $123.6(3)$ & $\mathrm{SI} 2-\mathrm{C} 21-\mathrm{C} 27 \mathrm{~A}-\mathrm{C} 27$ & $-8.7(4)$ \\
\hline CL1-NI1-C17A-C17 & $43.5(3)$ & $\mathrm{NI} 2-\mathrm{C} 21-\mathrm{C} 27 \mathrm{~A}-\mathrm{C} 27$ & $121.0(3)$ \\
\hline $\mathrm{P} 1-\mathrm{NI} 1-\mathrm{C} 17 \mathrm{~A}-\mathrm{C} 17$ & $-71.8(3)$ & $\mathrm{C} 22-\mathrm{C} 21-\mathrm{C} 27 \mathrm{~A}-\mathrm{C} 23 \mathrm{~A}$ & $5.9(3)$ \\
\hline $\mathrm{C} 13 \mathrm{~A}-\mathrm{NI} 1-\mathrm{C} 17 \mathrm{~A}-\mathrm{C} 17$ & $-113.9(3)$ & $\mathrm{SI} 2-\mathrm{C} 21-\mathrm{C} 27 \mathrm{~A}-\mathrm{C} 23 \mathrm{~A}$ & $173.66(19)$ \\
\hline $\mathrm{C} 13-\mathrm{NI} 1-\mathrm{C} 17 \mathrm{~A}-\mathrm{C} 13 \mathrm{~A}$ & $-36.15(18)$ & $\mathrm{NI} 2-\mathrm{C} 21-\mathrm{C} 27 \mathrm{~A}-\mathrm{C} 23 \mathrm{~A}$ & $-56.65(19)$ \\
\hline $\mathrm{C} 12-\mathrm{NI} 1-\mathrm{C} 17 \mathrm{~A}-\mathrm{C} 13 \mathrm{~A}$ & $-81.07(19)$ & $\mathrm{C} 22-\mathrm{C} 21-\mathrm{C} 27 \mathrm{~A}-\mathrm{NI} 2$ & $62.58(17)$ \\
\hline $\mathrm{C} 11-\mathrm{NI} 1-\mathrm{C} 17 \mathrm{~A}-\mathrm{C} 13 \mathrm{~A}$ & $-122.5(2)$ & $\mathrm{SI} 2-\mathrm{C} 21-\mathrm{C} 27 \mathrm{~A}-\mathrm{NI} 2$ & $-129.7(2)$ \\
\hline $\mathrm{CL} 1-\mathrm{NI} 1-\mathrm{C} 17 \mathrm{~A}-\mathrm{C} 13 \mathrm{~A}$ & $157.45(16)$ & $\mathrm{C} 23-\mathrm{NI} 2-\mathrm{C} 27 \mathrm{~A}-\mathrm{C} 27$ & $149.9(4)$ \\
\hline P1-NI1-C17A-C13A & $42.1(2)$ & $\mathrm{C} 22-\mathrm{NI} 2-\mathrm{C} 27 \mathrm{~A}-\mathrm{C} 27$ & $-164.4(4)$ \\
\hline $\mathrm{C} 13-\mathrm{NI} 1-\mathrm{C} 17 \mathrm{~A}-\mathrm{C} 11$ & $86.34(17)$ & $\mathrm{C} 21-\mathrm{NI} 2-\mathrm{C} 27 \mathrm{~A}-\mathrm{C} 27$ & $-123.3(4)$ \\
\hline $\mathrm{C} 12-\mathrm{NI} 1-\mathrm{C} 17 \mathrm{~A}-\mathrm{C} 11$ & $41.43(15)$ & $\mathrm{CL} 2 \mathrm{~A}-\mathrm{NI} 2-\mathrm{C} 27 \mathrm{~A}-\mathrm{C} 27$ & $-33.4(4)$ \\
\hline $\mathrm{CL} 1-\mathrm{NI} 1-\mathrm{C} 17 \mathrm{~A}-\mathrm{C} 11$ & $-80.05(14)$ & $\mathrm{P} 2-\mathrm{NI} 2-\mathrm{C} 27 \mathrm{~A}-\mathrm{C} 27$ & $79.2(4)$ \\
\hline $\mathrm{P} 1-\mathrm{NI} 1-\mathrm{C} 17 \mathrm{~A}-\mathrm{C} 11$ & $164.60(11)$ & $\mathrm{CL} 2 \mathrm{~B}-\mathrm{NI} 2-\mathrm{C} 27 \mathrm{~A}-\mathrm{C} 27$ & $-48.5(4)$ \\
\hline $\mathrm{C} 13 \mathrm{~A}-\mathrm{NI} 1-\mathrm{C} 17 \mathrm{~A}-\mathrm{C} 11$ & $122.5(2)$ & $\mathrm{C} 23 \mathrm{~A}-\mathrm{NI} 2-\mathrm{C} 27 \mathrm{~A}-\mathrm{C} 27$ & $113.8(4)$ \\
\hline $\mathrm{C} 25-\mathrm{C} 24-\mathrm{C} 23 \mathrm{~A}-\mathrm{C} 27 \mathrm{~A}$ & $1.0(5)$ & $\mathrm{C} 23-\mathrm{NI} 2-\mathrm{C} 27 \mathrm{~A}-\mathrm{C} 23 \mathrm{~A}$ & $36.2(2)$ \\
\hline $\mathrm{C} 25-\mathrm{C} 24-\mathrm{C} 23 \mathrm{~A}-\mathrm{C} 23$ & $178.8(4)$ & $\mathrm{C} 22-\mathrm{NI} 2-\mathrm{C} 27 \mathrm{~A}-\mathrm{C} 23 \mathrm{~A}$ & $81.8(2)$ \\
\hline $\mathrm{C} 25-\mathrm{C} 24-\mathrm{C} 23 \mathrm{~A}-\mathrm{NI} 2$ & $94.2(5)$ & $\mathrm{C} 21-\mathrm{NI} 2-\mathrm{C} 27 \mathrm{~A}-\mathrm{C} 23 \mathrm{~A}$ & $122.9(3)$ \\
\hline $\mathrm{C} 22-\mathrm{C} 23-\mathrm{C} 23 \mathrm{~A}-\mathrm{C} 27 \mathrm{~A}$ & $-7.8(3)$ & $\mathrm{CL} 2 \mathrm{~A}-\mathrm{NI} 2-\mathrm{C} 27 \mathrm{~A}-\mathrm{C} 23 \mathrm{~A}$ & $-147.1(2)$ \\
\hline $\mathrm{NI} 2-\mathrm{C} 23-\mathrm{C} 23 \mathrm{~A}-\mathrm{C} 27 \mathrm{~A}$ & $58.9(2)$ & $\mathrm{P} 2-\mathrm{NI} 2-\mathrm{C} 27 \mathrm{~A}-\mathrm{C} 23 \mathrm{~A}$ & $-34.5(3)$ \\
\hline $\mathrm{C} 22-\mathrm{C} 23-\mathrm{C} 23 \mathrm{~A}-\mathrm{C} 24$ & $174.3(3)$ & $\mathrm{CL} 2 \mathrm{~B}-\mathrm{NI} 2-\mathrm{C} 27 \mathrm{~A}-\mathrm{C} 23 \mathrm{~A}$ & $-162.2(2)$ \\
\hline $\mathrm{NI} 2-\mathrm{C} 23-\mathrm{C} 23 \mathrm{~A}-\mathrm{C} 24$ & $-119.0(4)$ & $\mathrm{C} 23-\mathrm{NI} 2-\mathrm{C} 27 \mathrm{~A}-\mathrm{C} 21$ & $-86.7(2)$ \\
\hline $\mathrm{C} 22-\mathrm{C} 23-\mathrm{C} 23 \mathrm{~A}-\mathrm{NI} 2$ & $-66.7(2)$ & $\mathrm{C} 22-\mathrm{NI} 2-\mathrm{C} 27 \mathrm{~A}-\mathrm{C} 21$ & $-41.06(18)$ \\
\hline $\mathrm{C} 23-\mathrm{NI} 2-\mathrm{C} 23 \mathrm{~A}-\mathrm{C} 27 \mathrm{~A}$ & $-121.6(3)$ & $\mathrm{CL} 2 \mathrm{~A}-\mathrm{NI} 2-\mathrm{C} 27 \mathrm{~A}-\mathrm{C} 21$ & $89.98(16)$ \\
\hline $\mathrm{C} 22-\mathrm{NI} 2-\mathrm{C} 23 \mathrm{~A}-\mathrm{C} 27 \mathrm{~A}$ & $-79.7(2)$ & $\mathrm{P} 2-\mathrm{NI} 2-\mathrm{C} 27 \mathrm{~A}-\mathrm{C} 21$ & $-157.43(14)$ \\
\hline $\mathrm{C} 21-\mathrm{NI} 2-\mathrm{C} 23 \mathrm{~A}-\mathrm{C} 27 \mathrm{~A}$ & $-35.17(19)$ & $\mathrm{CL} 2 \mathrm{~B}-\mathrm{NI} 2-\mathrm{C} 27 \mathrm{~A}-\mathrm{C} 21$ & $74.9(2)$ \\
\hline $\mathrm{CL} 2 \mathrm{~A}-\mathrm{NI} 2-\mathrm{C} 23 \mathrm{~A}-\mathrm{C} 27 \mathrm{~A}$ & $43.6(2)$ & $\mathrm{C} 23 \mathrm{~A}-\mathrm{NI} 2-\mathrm{C} 27 \mathrm{~A}-\mathrm{C} 21$ & $-122.9(3)$ \\
\hline $\mathrm{P} 2-\mathrm{NI} 2-\mathrm{C} 23 \mathrm{~A}-\mathrm{C} 27 \mathrm{~A}$ & $157.01(18)$ & & \\
\hline
\end{tabular}

